\title{
The Principle of Conservation of Structural Aspect: Facilitating Visual Communication and Learning in Organic Chemistry Instruction
}

john andraos

Submitted date: 17/05/2019 Posted date: 20/05/2019

Licence: CC BY-NC-ND 4.0

Citation information: andraos, john (2019): The Principle of Conservation of Structural Aspect: Facilitating Visual Communication and Learning in Organic Chemistry Instruction. ChemRxiv. Preprint.

An effective pedagogical method is presented for the visual communication of chemical reactions learned in organic chemistry undergraduate courses. The basis for the method is the preservation of the visual aspect of reactant and product structures so that the tracking of cleaved and formed chemical bonds is made self-evident. This consequently leads to improved clarity of presentation and a better understanding and grasp of proposed reaction mechanisms to explain product outcomes. The method is demonstrated for a variety of individual reaction types and synthesis plans. Various visual training exercises are also presented using ChemDraw Ultra 7.0 software and literature table of contents (TOC) graphics appearing in journal articles.

File list (2)

manuscript.pdf (746.43 KiB)

view on ChemRxiv - download file 
The Principle of Conservation of Structural Aspect: Facilitating Visual Communication and Learning in Organic Chemistry Instruction

John Andraos

CareerChem

504-1129 Don Mills Road

Toronto, ON M3B 2W4

Canada

c1000@careerchem.com

\begin{abstract}
An effective pedagogical method is presented for the visual communication of chemical reactions learned in organic chemistry undergraduate courses. The basis for the method is the preservation of the visual aspect of reactant and product structures so that the tracking of cleaved and formed chemical bonds is made self-evident. This consequently leads to improved clarity of presentation and a better understanding and grasp of proposed reaction mechanisms to explain product outcomes. The method is demonstrated for a variety of individual reaction types and synthesis plans. Various visual training exercises are also presented using ChemDraw Ultra 7.0 software and literature table of contents (TOC) graphics appearing in journal articles.
\end{abstract}

Keywords: upper-division undergraduate, curriculum, organic chemistry pedagogy, problem solving, organic synthesis

\title{
Introduction
}

It is well known that when students encounter organic chemistry for the first time in undergraduate courses, they perceive the subject as "hard". From their point of view their complaint stems from two major issues: (a) the apparent overwhelming volume of material that needs to be digested and learned in a relatively short period of time 
(typically a three-month course session); and (b) the material is presented in a visual manner that is not always "easy" or evident to understand what is going in any given chemical reaction that is presented to them. The inevitable outcome is increased frustration and ultimately a dislike for the subject for the majority of students, particularly those that take organic chemistry as a standard mandatory requirement for other disciplines such as pre-medical studies and life sciences. Instructors, on the other hand, teach the subject mainly by following a recommended course textbook that they themselves have not written and by assigning problems and exercises that they have not made up themselves. The evolution of textbooks has largely focussed on supplying a plethora of colour graphics and side bar instructional vignettes via accompanying electronic multimedia such as videos and visual software packages. However, the categorization of content according to functional groups, their physical and chemical properties, and their transformations to other functional groups has remained the same for over a century. Throughout the pedagogical process both instructors and textbook writers teach by a process of "osmosis" where the student views what the instructor writes on a blackboard or shows on a PowerPoint presentation and the student is required to mimic what he or she "sees". Learning takes place by a monkey-see-monkey-do approach where intense rote repetition and rote memorization is emphasized in the hope that students will eventually "get it", but with little if any real understanding or reasoning of what is going on. The mechanics of how to problem solve is rarely made explicit over the course of the instruction. Completely absent from the pedagogy is how to draw chemical structures in a clear manner. Moreover visual training exercises such as mental rotation and reflection are not incorporated into the syllabus though there is a substantial literature available on this subject [1-9] mainly conducted by workers in the cognitive and vision sciences. Surprisingly, none of this literature addresses the pedagogical problems that ensue when the preservation of structural aspect is not adhered to in drawing out chemical reactions. Nevertheless, the student sentiments and instructor challenges described have not changed despite a very long track record of discussion to better deliver course content, to improve teaching outcomes, and to improve the overall student-teacher experience in organic chemistry courses [10]. These problems have existed since the inception of organic chemistry instruction and it is expected that the 
situation will worsen as the knowledge base of discovery of organic reaction transformations continues to increase without limit. Given this state of affairs, one may ask what is the root cause of this dilemma and how can the situation be remedied, if at all?

The first thing to recognize and state is that the language of organic chemistry is entirely visual in the same sense as the language of pictorial hieroglyphics used by the Pharaohs of Ancient Egypt. Being able to both draw and read chemical structure drawings is therefore paramount to communicating and understanding chemical reactions in organic chemistry. When a child learns to read a language he or she must first recognize the shapes of the letters of the alphabet and then the shapes of words. Once these are mastered then the meanings of words can be interpreted and understood which in turn results in comprehension of whole sentences, thoughts, and stories. Similarly, when a child learns to count he or she first recognizes the shapes of the individual digits 0 to 9 and then the shapes of arithmetic operators,,$+- \times$, and $\div$ standing for addition, subtraction, multiplication, and division respectively. The understanding of abstract mathematical ideas is greatly facilitated and made more concrete by visual or geometric representations of algebraic concepts. Hence, basic arithmetic operations are visually described using familiar pictures of objects such as apples and oranges in arrays of columns and rows. By analogy, when an undergraduate student learns organic chemistry he or she learns to recognize various aspects of shapes of chemical structures such as element symbols for atoms, lines for chemical bonds, functional groups, conformations, electrophilic and nucleophilic sites, and acidic and basic sites. A student needs to learn how to draw and "read" the pictures of chemical structures before understanding what is going on in a transformation that begins with reactant chemical structures and ends with product chemical structures. In other words, the understanding of the chemical outcome of an organic chemical reaction is achieved by first mastering the visual code of the subject of organic chemistry which is the lingua franca of the subject.

In this work we put forward a concept called the principle of conservation of structural aspect which we introduce as a guiding principle in depicting chemical reactions and synthesis schemes which addresses the pedagogical challenges discussed. We have successfully implemented it over the last decade in tutorial sessions running in 
parallel to conventional courses in organic chemistry in second and third year undergraduate study at York University and Ontario Tech University. The aim is to draw out chemical equations in such a way that they can be visually read without need for unnecessary mental gymnastics such as reflections, rotations, and translations of chemical structures. This higher level of clarity in communication inevitably speeds up the understanding of what bond changes are occurring in a given transformation. The principle may be stated as follows. The principle of conservation of structural aspect states that the picture of the structure of a reaction product appearing in a balanced chemical equation has the same aspect or orientation as the picture of the structure of its progenitor substrates. Or, conversely the aspect of the pictures of the reactants is consistent with that of the reaction product of interest so that an atom-by-atom and a bond-by-bond comparison between these structures can be readily made. We contend that it is precisely the failure of adopting this practice of consistent display of structural aspect in instructional textbooks and research journal articles that causes untold anguish among students in understanding and seeing what is going on when they look at a drawing for the first time of a chemical transformation involving chemical structures. This, in turn, leads directly to significant impediments in understanding reaction mechanism fundamentals and implementing the central diagrammatic crux of depicting them; namely, the direction of electron flow using curly arrow notation. Pedagogical challenges surrounding the concept of curly arrow notation in the context of introductory organic chemistry instruction have been well documented [11-16]. When a visual equation is read from left to right, that is, from the reactant side to the product side, it must be made obvious as far as possible to show which parts of the starting structures remain unchanged and which are different as a consequence of the transformation when comparing the reactant structures with the product structures. Advantages of such an approach applied to individual reactions include: (1) the ability to easily map atoms of starting materials onto the structures of products; (2) better identification of target bonds formed in the product structure; (3) better identification and description of ring construction strategies; (4) higher probability of identifying reaction by-products; and (5) immediate elucidation of likely reaction mechanism pathways leading to observed products. When the principle of conservation of structural aspect is adopted for 
displaying schemes of synthesis plans it enhances their aesthetic presentation as well as it improves and speeds up the process of understanding and seeing what synthesis strategies are employed in a given plan. The method used to depict a synthesis scheme that employs a consistent structural aspect throughout is to choose a suitable structural aspect for the target product and work backwards step by step following the structures of the intermediates drawn with the same consistent aspect all the way back to the starting substrate. When read in the forward direction such a scheme is very easy to read visually, thus greatly facilitating target bond mapping and elucidating any ring construction strategies employed. In fact, a well-drawn scheme for a synthesis plan according to the principle of conservation of structural aspect does not require accompanying words to explain what is happening from step to step. In other words the drawings of the chemical structures appearing in the scheme should speak for themselves.

In this work we describe various visualization exercises that train students to draw out individual chemical equations and synthesis schemes following the principle of conservation of structural aspect. We begin with a preliminary series of visual training exercises involving mental rotation and mental reflection assisted by ChemDraw software that is ubiquitously available to chemistry students. This will serve to train students to redraw reactions appearing in the chemistry literature that do not follow the preservation of structural aspect from reactants to products, as will be described later. Next, we apply the conservation principle to the fundamental categories of reactions encountered in organic chemistry: additions, fragmentations, substitutions, redox reactions, rearrangements, and combinations of these in tandem and one-pot multi-component reactions. Close examination of these categories indicates a kind of visual algebra associated with each reaction type. Addition or aufbau reactions including couplings and condensations involve either the production of linear or cyclic products. The visual operator is obviously addition, or construction, in the same sense as algebraic addition with pluses and minuses corresponding to electrophilic and nucleophilic sites respectively that come together to form new chemical bonds. These are often called skeletal building reactions. Fragmentation, elimination, or abbau reactions involve splitting the starting substrate structure into one or more pieces. This is the exact reverse of addition. The visual operator is therefore subtraction or pruning. Substitution reactions involve 
exchange of one functional group for another in the starting substrate structure. The visual operator is exchange or metathesis. Rearrangement reactions involve a reshuffling of atom connectivity in the starting substrate structure or in a transient intermediate structure over the course of the reaction. The visual operator is connectivity scrambling. Redox reactions are either oxidation reactions with respect to the substrate of interest, or reduction reactions with respect to the substrate of interest. Oxidation reactions can be variously described as the addition of oxygen atoms to a substrate structure, the removal of hydrogen atoms from a substrate structure, or the electronic transformation of an atom in a substrate structure from being electron rich to being electron deficient (that is, electrons have been removed from that atom). Reduction reactions, on the other hand, can be variously described as the addition of hydrogen atoms to a substrate structure, the removal of oxygen atoms from a substrate structure, or the electronic transformation of an atom in a substrate structure from being electron deficient to being electron rich (that is, electrons have been added to that atom). This category of reactions is often characterized by the kind of reagents that operate on the substrate structure, whether they are oxidizing agents or reducing agents. For the majority of reactions in this category there is no formal visual operator associated with it since the skeletal arrangement of the substrate and product structures remains constant. Exceptions to this are insertions of oxygen atoms into the carbon skeleton to form rings such as epoxides (e.g., Prilezhaev reaction) or lactones (e.g., Baeyer-Villiger oxidation). Such reactions may be viewed as addition oxidation reactions. For each of these five reaction types the associated mechanisms are also drawn in a stepwise manner where the aspect of all structures is preserved throughout all elementary steps. Once drawing of all of these reaction types is mastered, students are then asked to examine table of contents (TOC) graphics appearing in journal articles that describe individual reactions and then to observe if authors depicted their chemical drawing according to the principle of structural aspect. If it is found that authors drew their structures in a haphazard manner that visually confuses the reader, then the students are tasked to redraw it along with highlighting the target bonds made in the product structure. Next, students are given synthesis schemes appearing in the literature and are tasked to redraw them, if necessary, according to the preservation of structural aspect applied consistently from the target product structure and throughout all 
of the intervening intermediate structures leading all the way back to the starting substrate structures in the first step. We describe each of these kinds of exercises in the following sections with illustrative examples. The Supporting Information contains additional exercises based on literature TOC graphics and synthesis plans that instructors can use in their own organic chemistry courses.

\section{Mental Rotation and Mental Reflection}

Visual exercises involving mental rotation and reflection were put forward by workers in the cognitive, behavioural, and perception sciences. The most familiar visual tool used to test human subjects in this task is the Shepard-Metzler diagram (SMD) [17]. Schemes 1 and 2 show example SMDs created using ChemDraw Ultra 7.0 for mental rotation and mental reflection exercises. Students are given such diagrams and then they explore the various object tools available in ChemDraw that can be used to manipulate them. The "flip horizontal" command $(\mathrm{Shift}+\mathrm{Ctrl}+\mathrm{H})$ reflects the object in the $y$-axis. The "flip vertical" command (Shift+Ctrl+V) reflects the object in the $x$-axis. The "rotate" command $(\mathrm{Ctrl}+\mathrm{R})$ rotates the object to a user specified number of degrees. Clockwise rotations are entered as positive angles with respect to the vertical and anticlockwise rotations are entered as negative angles with respect to the vertical. Next, students are given three SMDs and are asked to select the pair that are either mirror images or are identical. Or, students are given two SMDs and are asked to describe their relationship. Example exercises are shown in Schemes 3 and 4. In Scheme 3 images A and $B$ are identical. In Scheme 4 the two images are mirror images since a $120^{\circ}$ anticlockwise rotation in the plane of the paper of the second SMD results in an image that is the mirror image of the first SMD (see Scheme 1). Students then repeat these exercises using images of glassware also available in ChemDraw such as flasks and condensers. Once students have trained their mind's eye with these visualization exercises they apply their skills on images of chemical structures. A summary of key transformations is shown in Scheme 5 using stereochemistry as a structural feature in the chemical drawing. From Scheme 5 students are able to deduce the following patterns: (a) any reflection operation applied to a stereochemical figure results in an image which is enantiomeric to 
the original; (b) any rotational operation applied to a stereochemical figure results in an image which is identical to the original; (c) $180^{\circ}$ clockwise rotations of a figure in a given plane or about a given axis lead to figures that are identical to $180^{\circ}$ anti-clockwise rotational transformations; (d) "flip vertical" is identical to "mirror image along $x$-axis drawn above or below figure"; and (e) "flip horizontal" is identical to "mirror image along $y$-axis drawn to the right or left of the figure". It should be noted that drawings appearing with the words "mirror image" in Scheme 5 are drawn from scratch without using any object tools. This exercise can be extended to include planar aromatic structures and chair conformations of six-membered rings as given in the Supporting Information.

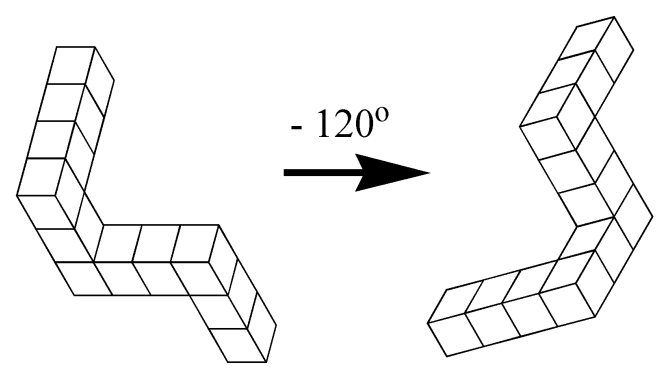

ChemDraw

$\Rightarrow$ Object

$\Rightarrow$ Rotate

Scheme 1. Example Shepard-Metzler diagram used in mental rotation visual exercises.
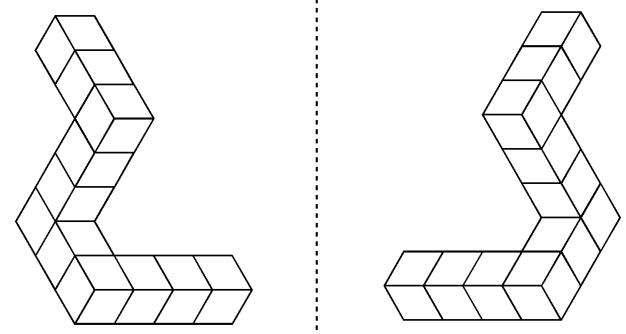

ChemDraw

Object

$\Rightarrow$ flip horizontal

Scheme 2. Example Shepard-Metzler diagram used in mental reflection visual exercises. 


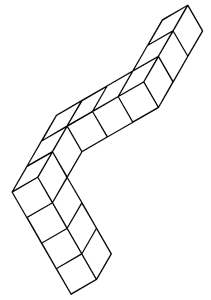

A

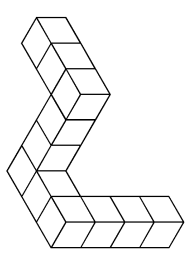

B

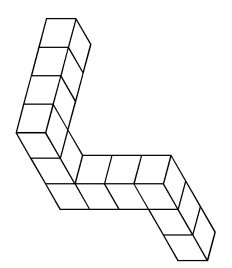

C

Scheme 3. Pairwise relationship exercise using Shepard-Metzler diagrams. Which pair of images is identical?
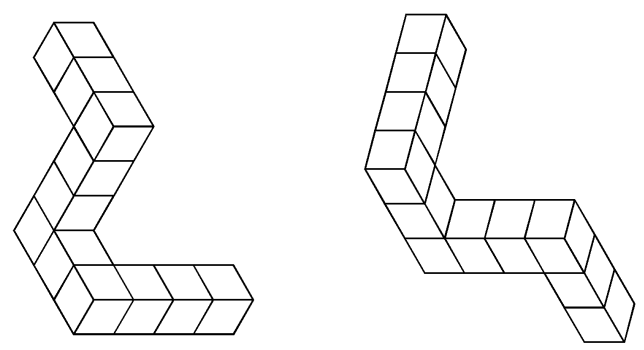

Scheme 4. Pairwise relationship exercise using Shepard-Metzler diagrams. How are these images related?

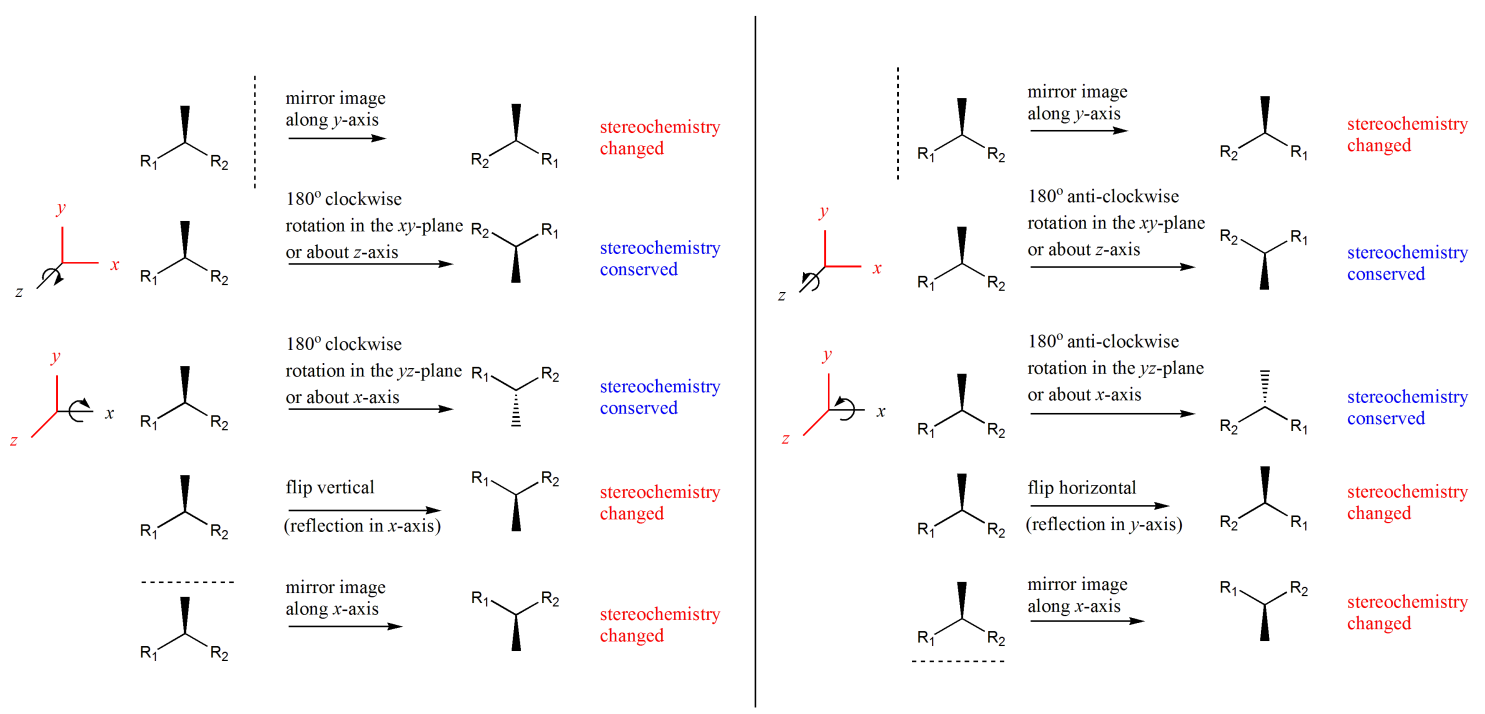

Scheme 5. Example of a stereochemical structure that is subjected to various object manipulations.

\section{Organic Reaction Types}

Depending on various compilations $[18,19]$ of named organic reactions, the number of such reactions is in the neighbourhood of at least 600 . Though this may be a large number they can be conveniently classified according to six reaction types: 
additions, eliminations, substitutions, rearrangements, redox reactions, and combinations of these in tandem or one-pot transformations. In some cases, a given reaction can be categorized in more than one of these headings. An example is the Grignard reaction which can be classified as an addition or as a redox reaction since the magnesium metal $\left(\mathrm{Mg}^{0}\right)$ is oxidized to a magnesium salt $\left(\mathrm{Mg}^{+2}\right)$ by-product and concomitantly the aryl or alkyl halide carbon atom is reduced from $\mathrm{a}+1$ state to $\mathrm{a}-1$ state in forming the Grignard reagent. What is important to point out is that though the number of new organic transformations continues to increase as discoveries are continuously made, they will always be classifiable into at least one of these six categories. Nevertheless, when any of the named organic reactions is introduced to students for the first time, the four key questions that need to be answered are:

Q1. Where are the starting materials in the product structure?

Q2. Where are the newly formed chemical bonds in the product structure?

Q3. What are the suggested by-products for the reaction based on the target bond map for the reaction?

Q3. What does the above information suggest about the possible mechanism for the reaction?

All of these questions can be answered with greater clarity and facility when the principle of conservation of structural aspect is applied. Scheme 6 shows examples of standard reactions in each of the categories encountered in the introductory organic chemistry curriculum. The left side of Scheme 6 shows a representation where the structural aspect between reactants and products is not preserved and the right side shows one in which it is. A quick comparative glance illustrates the obvious benefits of the standardized approach. Once students learn how to identify nucleophilic and electrophilic sites and acidic and basic sites in reactant structures, and identify the mapping of new chemical bonds created in the reaction in question, they can readily suggest mechanistic steps that would lead to the target products and better predict any reaction by-products. We observe unnecessary mental translation, rotation, and reflection operations that need to be done in the left-hand-side representations of all reactions shown in Scheme 6. The representations of the Wagner-Meerwein rearrangement and Biginelli reactions are particularly taxing on the eye when one sees them, as a student would, for the first time 
compared to the right-hand-side representations. In fact, graphical representations of rearrangement and multi-component reactions can be particularly improved for enhanced visual clarity when the principle of conservation of aspect is applied. In the case of the Wagner-Meerwein rearrangement reaction, the rearrangement of the secondary carbocation intermediate to the tertiary carbocation intermediate is clearly shown, as well as the two representations of the latter intermediate with respect to the substrate and product structures, respectively. The two juxtaposed representations of the same tertiary carbocation shown in the rectangle are very important to visualize how this reaction proceeds. In the case of the Biginelli reaction, the order and orientation of the three reagents appearing on the left-hand side of the equation are important in allowing the student to map out the atom connectivity and bonding in the product structure.

\section{Friedel-Crafts acylation (addition)}

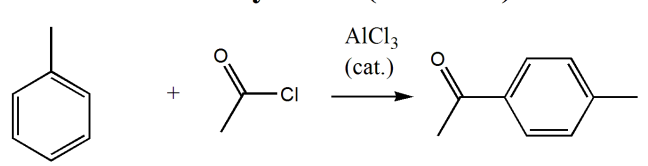

Aromatic nitration (substitution)

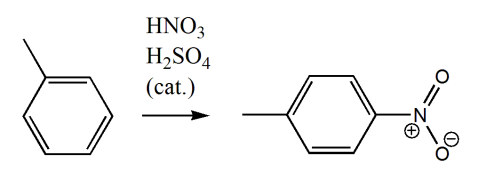

Hofmann degradation (elimination)

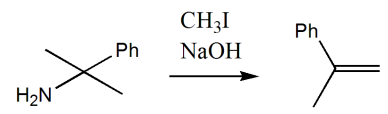

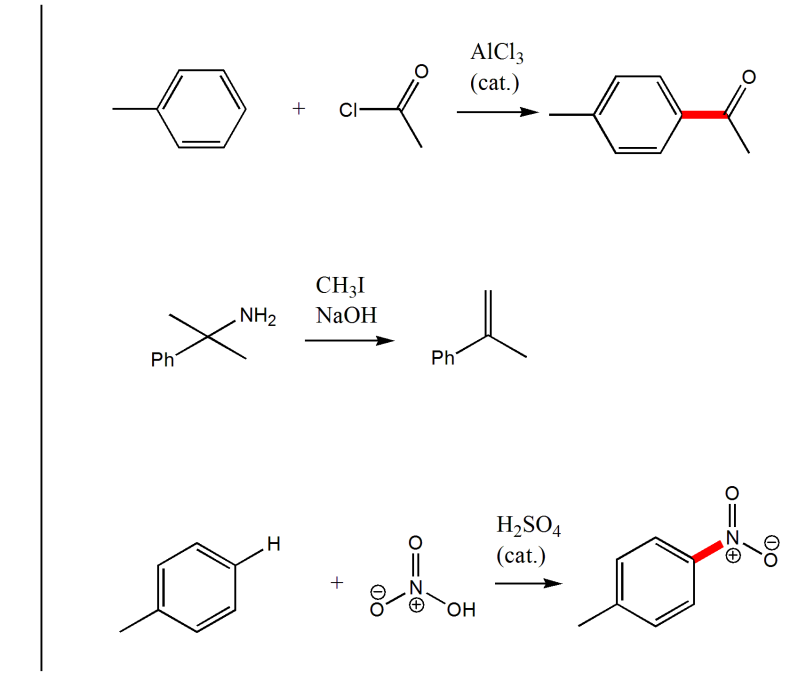

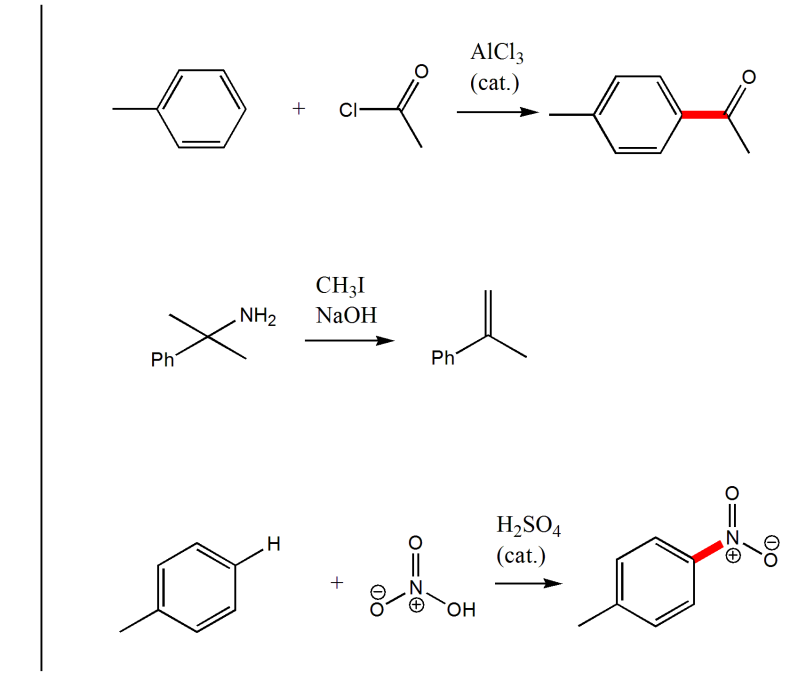


Wagner-Meerwein rearrangement
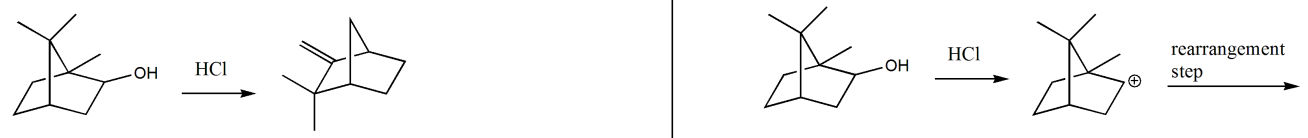

Baeyer-Villiger oxidation (additive)
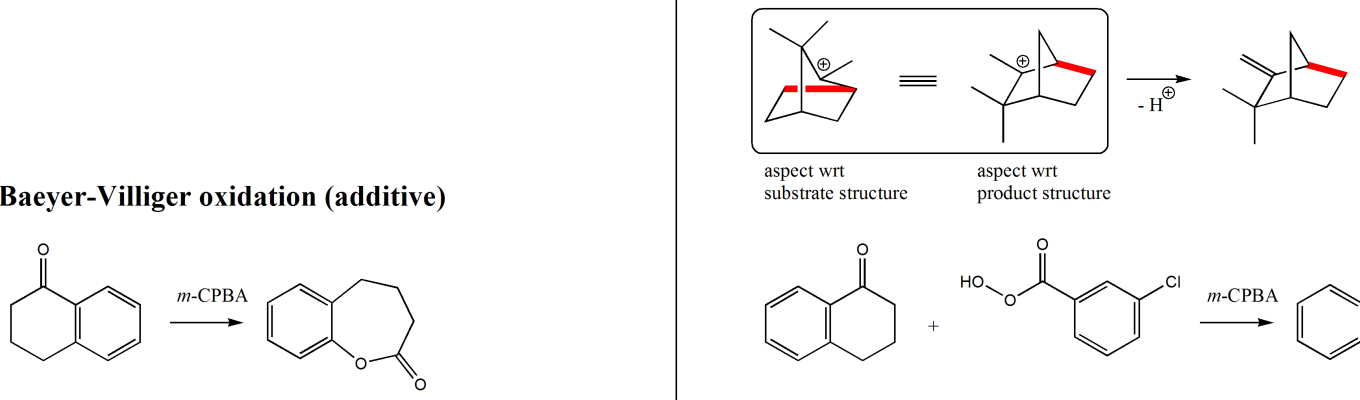

Biginelli reaction (multi-component)
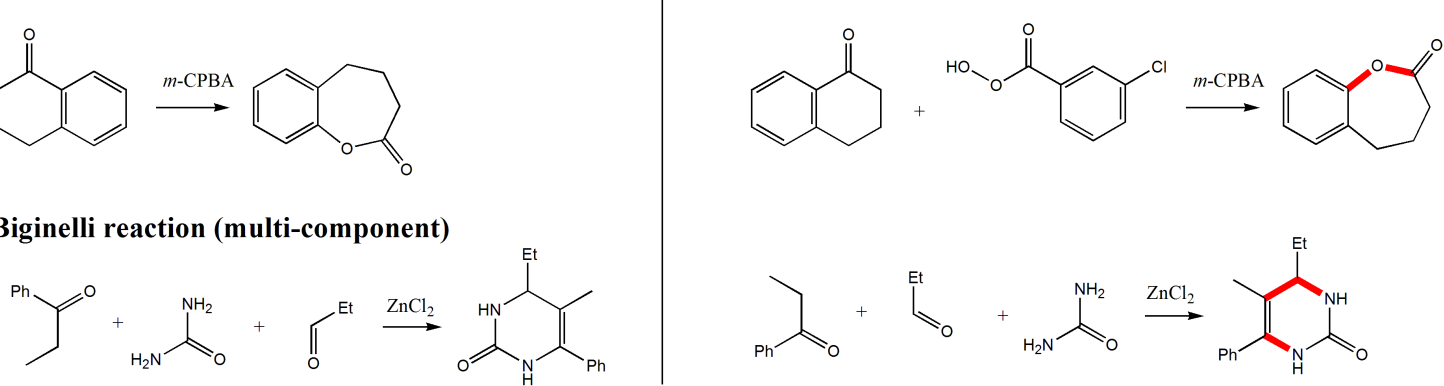

Scheme 6. Example standard named organic reactions in each of the six categories of reactions. The right side shows representations following the principle of structural aspect and the left side does not.

In Scheme 7 we juxtapose two representations of the same [3+2] cyclization reaction [20]. In the first representation given by the authors, we note differences in the graphical representations of the phenyl groups between reactant and product structures, and orientation changes in the graphical representation of the acid chloride reagent and in the direction of the $\mathrm{C}=\mathrm{S}$ bond in the thioketone reagent when compared to its direction in the product structure. All of these visual problems are eliminated in the second representation of the reaction and hence, understanding of how the target product is formed is significantly enhanced. 
(A)<smiles>[Y]C(=O)CCCCC(=O)Cl</smiles>

(B)<smiles>S=C(Nc1ccccc1)Nc1ccccc1</smiles><smiles>O=C(Cl)CCl</smiles><smiles>O=C1CN(c2ccccc2)C(=S)N1c1ccccc1</smiles>

Scheme 7. Two representations of a [3+2] cyclization reaction [20].

Scheme 8 shows four representations of the same [4+2] cycloaddition reaction [20]. In representation A given by the authors we note the following visual problems: (a) the product structure has a distorted bond angle, and (b) the orientations of both reagent structures do not align with the orientation of the structure of the product. Clearly, the distorted bond angle in the product structure is unappealing, however it was imposed in order for the scheme to fit in a limited space on the page. This reason is often the one that explains many of the apparent visual distraction problems appearing in literature schemes of reactions and synthesis plans, since editors of journals and textbooks are fixated only on reducing costs by reducing page counts of articles and books while sacrificing effective visual communication. Representations B, C, and D follow the principle of conservation of structural aspect, however it is representation $\mathrm{C}$ that also fulfills the criterion of reduced space. Therefore, it is this representation that should have been chosen to represent the reaction in published form that satisfies both visual clarity and limited space criteria. 
(A)

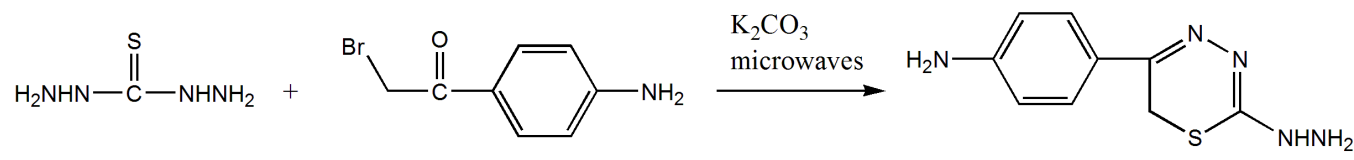

(B)<smiles>NNC(=S)NNNC(=O)CCCCCC(=O)c1ccc(N)cc1</smiles>

(C)

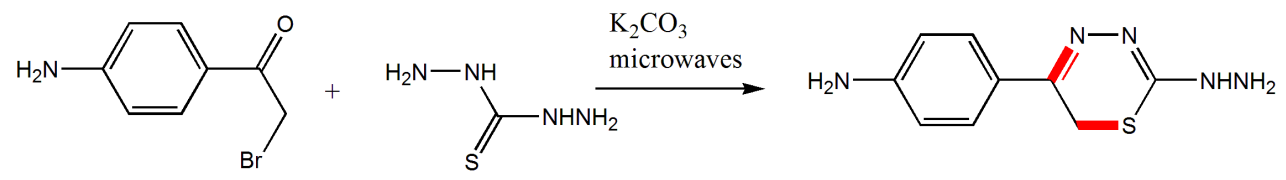

(D)<smiles>Nc1ccc(C(=O)CBr)cc1</smiles><smiles>NNC(=S)NN</smiles><smiles>NC1=NN=C(c2ccc(N)cc2)CS1</smiles>

Scheme 8. Four representations of a [4+2] cyclization reaction [20].

Scheme 9 shows two representations of the same Grob fragmentation reaction leading to cyclodec-5-enone [21]. Representation A nicely illustrates visual trickery when examining the relative positions of the $\mathrm{C}=\mathrm{C}$ and $\mathrm{C}=\mathrm{O}$ bonds in the product versus the relative positions of the hydroxyl and tosylate leaving group in the reactant, thus obscuring mechanistically how the fused bond cleaves in the reaction. On the other hand, representation $\mathrm{B}$ is much clearer in illustrating the entire process; however, the representation of the product structures shows a distortion in bond angles for the ketone group. Hence, numerical labeling of the carbon atoms is necessary to show that the distorted product structure in representation B is identical to the more visually pleasing product structure shown in representation A. This example is a nice demonstration of the visual jarring effect a graphical representation can have on a novice student viewer and what psychological impact this can have on their learning of organic chemistry and experience in the classroom. 
(A)

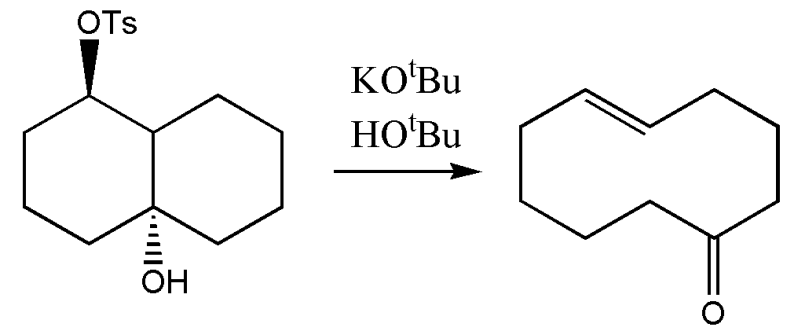

(B)<smiles>O[C@H]1CCC[C@@]2(O)CCCCC12</smiles><smiles>CC(C)(C)O[Mg]</smiles><smiles>CCCCC=C1CCCCC1=O</smiles><smiles>C#CCC=C1CCCCC1=O</smiles>
cyclodec-5-enone

Scheme 9. Two representations of a Grob fragmentation reaction [21].

Rearrangement reactions are the most challenging to represent according to the principle of conservation of structural aspect. It is obvious that the atom connectivity shuffling will necessarily result in drastically different representations of structures when comparing reactants with products. Scheme 10 shows an example acid-catalyzed methyl migration reaction given in a well-known textbook used in introductory organic chemistry courses [22]. Clearly, this representation camouflages what is occurring in the reaction since it is not immediately clear which methyl group is migrating and which methyl groups remain in the same position. Scheme 11 shows two different ways of depicting the reaction. In the first case, the aspect of the product matches that of the starting material shown in Scheme 10 and in the second case, the aspect of the product matches that of the product shown in Scheme 10. We can see clearly that there is an apparent visual "crossover" in the graphical representation shown in Scheme 10 which obscures the mechanics of the rearrangement of the intervening carbocation intermediate. From a pedagogical point of view Scheme 11 goes a long way in illustrating to the student how this reaction proceeds. Therefore, when representing rearrangement reactions mechanistically in an effective manner one needs to use the orientations of both the starting substrate and the final product simultaneously as was done in the 
representation of the Wagner-Meerwein rearrangement reaction shown earlier in Scheme 6. Over the course of writing out the mechanism the key intermediate which rearranges needs to be written in two ways: one representation maps onto the starting substrate structure and the other representation maps onto the product structure. Both representations of the same key intermediate need be written out and, if necessary, the carbon skeletons need to be labeled with symbols or numbers so that an atom-to-atom mapping can be made. This kind of atom labeling is particularly useful in tracking deepseated rearrangements such as Wagner-Meerwein (see Scheme 6), camphene, di- $\pi$ methane, and Nametkin rearrangements.<smiles>CC1=C(C)CC(C)C1</smiles>

Scheme 10. Representation of a [1,2]-methyl group migration rearrangement reaction [22].

\section{Aspect with respect to starting substrate}

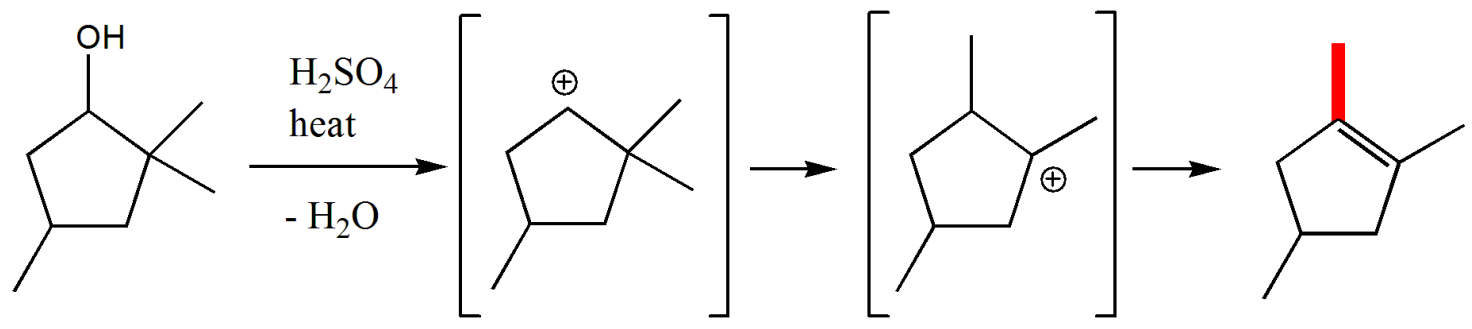

\section{Aspect with respect to product}

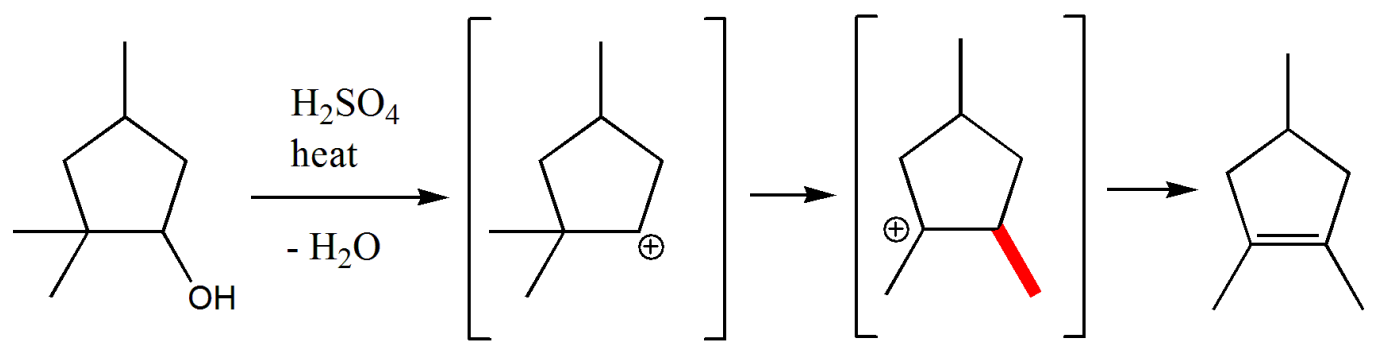


Scheme 11. Enhanced representation of a [1,2]-methyl group migration rearrangement reaction.

A particularly interesting rearrangement that illustrates extreme visual trickery in deciphering which group migrates is the acid-catalyzed cinenic acid to geronic acid rearrangement [23-27] shown in Scheme 12. In representations A and D, the diagrams visually suggest that the $\mathrm{COOH}$ group migrates from right to left and from left to right, respectively. However, in representations $\mathrm{B}$ and $\mathrm{C}$, the diagrams visually suggest that a methyl group migrates from left to right and from right to left, respectively. Hence, the way the structures of the reactant and the product are drawn relative to each other influences one's perception of which group is migrating! If one invokes that the $\mathrm{COOH}$ group migrates in representations $\mathrm{B}$ and $\mathrm{C}$, then a mental flipping of the reactant structure is required in representation $\mathrm{B}$ and a mental flipping of the product structure is required in representation C. Experimental evidence for this reaction indicates that the mechanism (see Scheme 13) follows an acid-catalyzed dehydration of the $\mathrm{COOH}$ group to an acylium ion which fragments to release carbon monoxide and yielding a dimethyl substituted tertiary carbocation. This cation is recaptured by carbon monoxide to regenerate the $\mathrm{COOH}$ group, but on the opposite side of the molecule. Hence, it is essentially the $\mathrm{COOH}$ group that migrated, not one of the methyl groups. 
(A)

(B)<smiles>CC1(C)CCCC(C)(C(=O)O)O1</smiles>

$\mathrm{H}^{+}$(cat.)<smiles>CC(=O)CCCC(C)(C)C(=O)O</smiles>

(C)<smiles>CC1(C)CCCC(C)(C(=O)O)O1</smiles>

$\mathrm{H}^{+}$(cat.)<smiles>CC(=O)CCCC(C)(C)C(=O)O</smiles>

(D)<smiles>CC1(C)CCCC(C)(C(=O)O)O1</smiles><smiles>CC(=O)CCCC(C)(C)C(=O)O</smiles>

Scheme 12. Various representations of the acid-catalyzed cinenic acid to geronic acid rearrangement reaction.

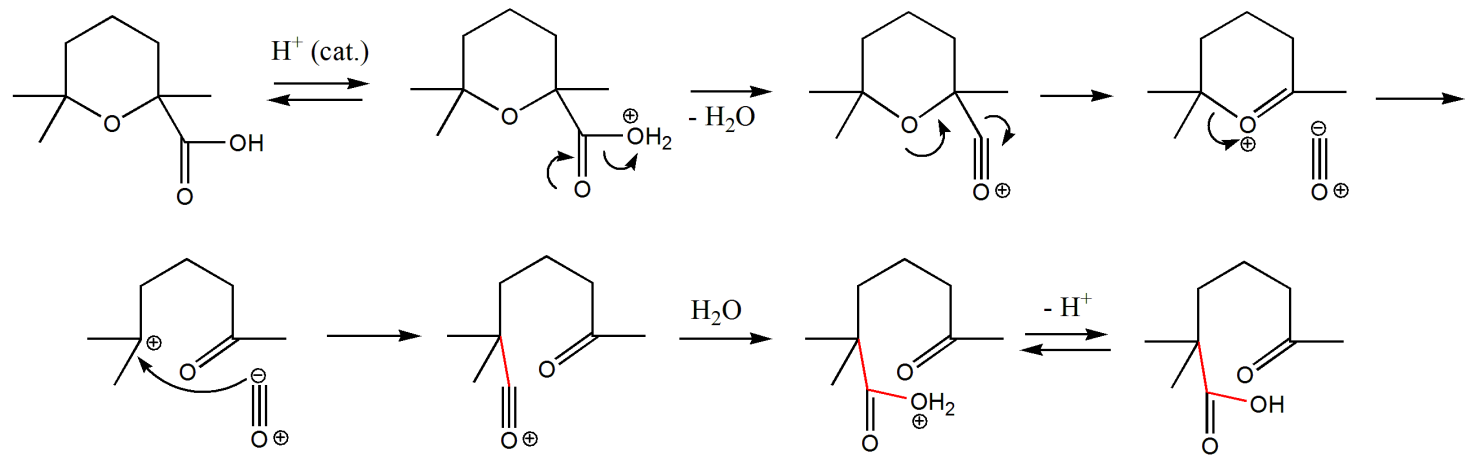


Scheme 13. Mechanism of the acid-catalyzed cinenic acid to geronic acid rearrangement reaction [23-27].

\section{Journal TOC Graphics}

After having gone through the preliminary visual gymnastics exercises using ChemDraw and then learning how to depict fundamental named organic reactions using the principle of conservation of structural aspect, students can then embark on applying the principle to transformations found in table of contents (TOC) graphics found in journal articles. The primary journals we have used to select examples are those that mainly publish communications such as Chemical Communications, Synthetic Communications, Synlett, Synthesis, Organic Letters, Tetrahedron Letters, Tetrahedron, and Tetrahedron Asymmetry. The Supporting Information contains several examples taken from Organic Letters between 2009 and 2013. What is emphasized here is that though the organic transformations selected are ones that students have never seen before in the standard repertoire of reactions encountered in the curriculum, they are still able to decipher them with confidence using the visual techniques they previously mastered. Firstly, they examine the display of a given reaction given by authors and use the authors' representation of the target product structure as a reference benchmark. Based on this pivot, students are tasked to draw out the structures of the reagents in the same aspect of that product structure and then to highlight the target bonds that were made. This exercise can actually be accomplished based on viewing the chemical structures as pictures and seeing what is constant and what is changing, in principle without knowledge of the actual chemistry. Of course, when nucleophilic, electrophilic, acidic, and basic sites are identified as a consequence of background chemical knowledge a more in-depth understanding is achieved beyond examining a reaction only from an artistic or perspective point of view. From our experience we have found that students' confidence and enjoyment of the subject increases by leaps and bounds when they are able to apply these techniques to reactions that are new and unfamiliar to them. Essentially they are training themselves to read, understand, and appreciate current cutting-edge research in the field. 
Of particular interest in this exercise are reactions that form rings since these are the most powerful kinds of reactions in synthetic organic chemistry methodology. The ring motif is found in almost all natural products and pharmaceuticals and hence any reactions discovered that can form them in a single step are highly interesting and highly prized. The exercise of deciphering the product target bond map is akin to working out the assembly of a molecular jigsaw puzzle that is very appealing from an intellectual point of view and quickly grabs students' attention. For illustrative purposes we demonstrate, in turn, four examples of such reactions taken from the literature that we have used in the classroom where the principle of conservation of structural aspect was implemented as a key tool to elucidate them mechanistically. In each case the representation designated as " $\mathrm{A}$ " is that of the original authors and the others are those where the principle of conservation of structural aspect was used. All target synthesis bonds appearing in the product structure are highlighted in bold type. Scheme 14 shows an example [3 + 3] cycloaddition of a propargyl alcohol and an aziridine under basic conditions [28]. In representation A the orientations of both reagents require adjusting as shown in representation B in order to map them directly onto the product structure. Though representation B shows distorted structures for the reagents, the payoff is that bond mapping onto the product structure is greatly facilitated. Representation $\mathrm{C}$ is a corrected version where bond distortions are eliminated, but the general orientation of reagents matches that of the product structure. Therefore, representation B can be thought of as an intermediate conceptual one that students use to formalize in their own minds how the product structure is formed. From a mechanistic standpoint, students deduce the following molecular events once representation B is written down. The base tert-butoxide deprotonates the alcohol and the resulting alkoxide nucleophilically attacks the carbon atom of the aziridine ring bearing the $\mathrm{R}_{2}$ group. Next, the resulting $\mathrm{N}^{-}$ nucleophile attacks the beta-carbon atom of the alkyne group to complete the [3+3] cyclization. The final product structure is obtained after two proton transfer steps. Based on this sequence of elementary steps, students deduce that there are no net by-products formed and so the reaction is $100 \%$ atom economical. Scheme 15 shows a threecomponent coupling reaction leading to a [3+2+1] cyclization [29]. In representation A the aryl iodide reagent is the only one that can be mapped directly onto the product 
structure; the carbon monoxide is put in the wrong position on the reactant side relative to the other two reagents, and the $\alpha, \beta$-unsaturated ketone reagent appears upside down. Representation B corrects these visual problems and it is now readily apparent where the target synthesis bonds are in the product structure. Upon comparing the left- and righthand sides of the chemical equation, it is clear that the iodine atom and one of the COOEt groups are lost from the aryl reagent. Once students deduce that palladium acetate, Xphos, triethylamine, and THF represent the catalyst, ligand, base, and reaction solvent respectively, they are able to conjecture the by-product outcomes of the reaction as triethylammonium iodide, carbon dioxide, and ethanol after an aqueous workup. This result can be deduced without explicit knowledge of the reaction mechanism. Scheme 16 shows a thermal cyclization reaction that produces a fused [4.3.0] bicyclic ring system [30]. Representation A shows the starting chromium Fischer carbene precursor in a linear geometry that obscures how the cyclization takes place. Moreover, the authors further confuse the reader by depicting the cyclohexane ring explicitly in the structure of the reagent but not in the product structure. Representation B redraws the starting material in the same aspect as the product and also shows the structure of the intermediate carbene that inserts into the two $\pi$-bonds. In this case, the by-products of the reaction are chromium tricarbonyl and two equivalents of carbon monoxide. The product bond mapping indicates that two rings are formed simultaneously, a $(6+0)$ cyclization creates the piperidine ring and a $(4+1)$ cyclization creates the cyclopentane ring. Since both rings are made simultaneously each of these cyclizations is written in parentheses and the whole transformation is written in square brackets as shown in the notation given in the caption of Scheme 16. Note that the representation of the spectator cyclohexane ring appears the same in both the substrate and product structures. In these three examples, the target bond mapping immediately allows students to deduce the ring construction strategies employed for the reactions using the notation shown, and also the occurrence of reaction by-products so that a fully balanced chemical equation can be written down for each transformation. Scheme 17 shows a base-catalyzed rearrangement reaction involving a ring expansion reaction to form a fused [4.2.0] bicyclic ring system [31]. In representation $A$ the authors used colour coding to show that the $\beta$-lactam ring moiety cleaves (shown in red in the substrate structure) and a cyclobutane ring moiety 
forms (shown in blue in the product structure). Rotational adjustment of the substrate structure in representation B makes more clear these ring breaking and ring forming events since it shows a direct mapping of atoms from the substrate structure onto the product structure. A check of the element count in both structures indicates that the overall transformation is a rearrangement that produces no by-products. Representation $\mathrm{C}$ shows two possible mechanisms depending on the order of base-catalyzed $\beta$-lactam cleavage and $[2+2]$ cyclization. In the first mechanism the $[2+2]$ cyclization occurs before $\beta$-lactam cleavage whereas in the second mechanism the sequence is reversed. The second mechanism seems more plausible since it avoids the highly strained tricyclic intermediate shown in the first mechanism.

(A)<smiles>[R]C1C([R])N1[AsH]</smiles><smiles>OCC#C[Te]</smiles>

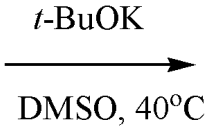<smiles>[R]C1C([R])N1[AsH]</smiles>

(B)

(C)
$t$-BuOK
$t$-BuOK

DMSO, $40^{\circ} \mathrm{C}$<smiles></smiles>

DMSO, $40^{\circ} \mathrm{C}$<smiles>[R2]C1OC=C(C[Al])N([Y5])C1[R]</smiles><smiles>[R2]C1OC=C(CBr)N([AsH])C1[R]</smiles>

Scheme 14. Example [3+3] cycloaddition reaction [28]. 
(A)

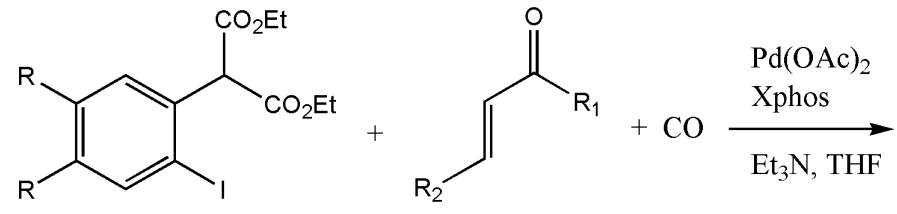<smiles>[R]C(=O)C1=C(O)c2cc([R])c([R])cc2C(C([R])=O)C1[R]</smiles>

(B)<smiles>[R]c1cc(I)c(C(C(=O)OC)C(=O)OCC)cc1[2H]</smiles><smiles>[R]C=CC([R])=O</smiles>

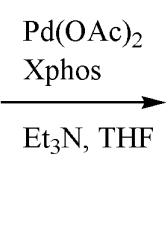<smiles>[R]C(=O)C1=C(O)c2cc([R])c([R])cc2[C@H](C([R])=O)[C@H]1[R]</smiles>

Scheme 15. Example [3+2+1] multi-component cycloaddition reaction [29].

(A)<smiles>C=C/C=C(\CCCN(/C=C\C(=O)OC(C)(F)F)CC1CCCCC1)C(=O)OC</smiles>

(B)

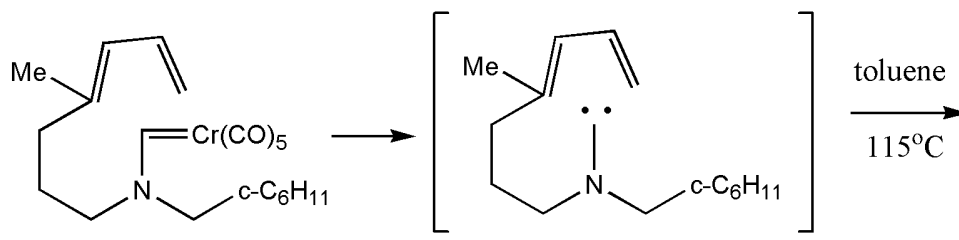<smiles>CC(C)CN1CCC[C@@]2(C)CCC[C@@H]12</smiles>

Scheme 16. Example $[(6+0)+(4+1)]$ intramolecular cyclization reaction [30]. 
(A)<smiles>C=CC1(C)CC(=O)N1C(=C)C</smiles>

(B)<smiles>C=CC1(C)CC(=O)N(C(=C)C)C1</smiles>

$\mathrm{C}_{9} \mathrm{H}_{13} \mathrm{NO}$ Mol. Wt.: 151

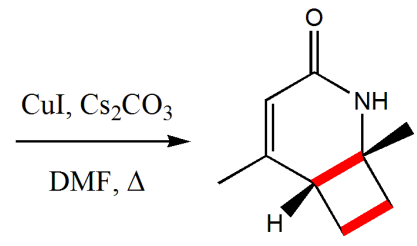

$\mathrm{C}_{9} \mathrm{H}_{13} \mathrm{NO}$
(C)<smiles>C=CC(C)(C)N1CC(C)(C)CC1=O</smiles>

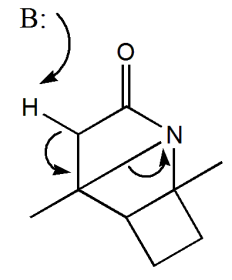<smiles>CC</smiles><smiles>CC1=CC(=O)NC2(C)CCC12</smiles>
$\stackrel{\oplus}{\mathrm{BH}}$<smiles>[B][C+](C)C</smiles>

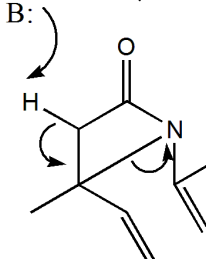<smiles>C=CC(C)=CC(=O)NC(C)(C)C</smiles><smiles>[B]C(C)C</smiles><smiles>C=C/C(C)=C\C(=O)NC(=C)C</smiles><smiles>CC1=CC(=O)NC2(C)CCC12</smiles>

Scheme 17. Example rearrangement reaction showing a ring expansion of a $\beta$-lactam to a fused [4.2.0] bicyclic ring system [31].

\section{Schemes for Synthesis Plans}

The final application of the conservation of structural aspect principle is demonstrated for synthesis plans since these represent the culmination of why students need to go through the preliminary visualization exercises while building up their mental vocabulary and toolbox of organic chemical reactions, and also what makes the subject of organic chemistry so interesting and creative. When faced with any scheme for a synthesis plan to a given target product the following questions need to be addressed: Q1. Where are the starting materials in the target product? Q2. Where are the newly formed chemical bonds in the target product structure? Q3. How to draw a bond map for the target product structure? Q4. How to parameterize ring construction strategy? 
We point out that these questions are very similar to the ones asked when addressing individual organic chemistry reactions. However, there is one important distinction. In the case of individual reactions producing rings, the ring mapping in the product structure and the ring strategy employed are one and the same. In the case of a synthesis plan composed of several reactions, ring mapping and ring strategy parameterization are not necessarily the same. For example, a product having a six-membered ring may have an overall ring mapping of [4+2] for example; however, this could be made either by a single [4 +2] cyclization strategy or by a stepwise construction sequence where a 4carbon fragment adds to a 2-carbon fragment in one step and then a $[6+0]$ cyclization occurs in a subsequent step. Both cases can be described as a [4+2] ring mapping in the product structure, but each is described by a different ring construction strategy. In the next section we illustrate these ideas using two examples of synthesis plans taken from the pedagogical and research literature, respectively.

Scheme 18 shows a diagram depicting the three-step synthesis of the pharmaceutical antipyrine taken from a venerable textbook on undergraduate laboratory experiments [32] where the principle of conservation of structural aspect is applied. Also shown are the reaction by-products in each step. The first step is a base-induced Claisen condensation of two equivalents of ethyl acetate followed by a [3+2] cyclization of the $\beta$-ketoester intermediate product with phenylhydrazine. The final step is a base-induced $N$-methylation reaction using dimethylsulfate. The ring mapping for the product structure is $[2+2+1]$; whereas, the ring construction strategy is $[3+2]$ occurring in step 2 . We note how facile Scheme 18 is to read in terms of tracking what bonds are made in each reaction step. By looking at the highlighted bonds in the antipyrine structure we can easily pinpoint which atoms of the reagents contributed to the product structure. The only reagent that did not contribute any atoms to the product structure is sodium metal. When we contrast Scheme 18 with a different representation of the same plan shown in Scheme 19, we see how all of the information described above is obscured and therefore hard to tease out. The following problems are observed: (a) the two equivalents of ethyl acetate needed in the first step are not explicitly shown; and the (b) the orientations of all intermediate products and final product are not uniform throughout. Hence, Scheme 18 is of higher pedagogical value than Scheme 19. It may be argued that Scheme 19 may be 
more appropriate to display in a competition examination rather than in a course textbook assuming that students taking the competition exam have been trained beforehand in visual manipulations as described in this work.<smiles>CCOC(=O)CCC(C)C(=O)OCC(C)=O</smiles>

$81 \%$<smiles>Cc1cc(=O)n(-c2ccccc2)[nH]1</smiles>

$\mathrm{NaOH}$

$(\mathrm{MeO})_{2} \mathrm{SO}_{2}$

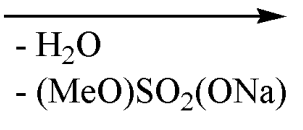

$74 \%$<smiles>Cc1cc(=O)n(-c2ccccc2)n1C</smiles>

antipyrine

Scheme 18. Synthesis plan for antipyrine [32] drawn according to the principle of conservation of structural aspect.

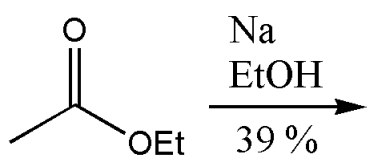<smiles>CCOC(=O)CC(C)=O</smiles><smiles>CNN</smiles><smiles>Cc1cc(=O)n(-c2ccccc2)[nH]1</smiles>

$\mathrm{NaOH}$ dimethyl sulfate<smiles>Cc1cc(=O)n(-c2ccccc2)n1C</smiles>

\section{antipyrine}

Scheme 19. Synthesis plan for antipyrine [32] drawn without regard to the principle of conservation of structural aspect. 
Scheme 20 shows a diagram for the synthesis of the natural product (+)-lycoridicine [33] as depicted by the authors. This literature example was also selected by $\mathrm{Wu}$ and Shah to study visuospatial thinking by students [2]. Though the display is very compact and was originally displayed in a single column in the standard two-column format of the journal article, we note a number of visual problems with this picture. The orientation and stereochemical depictions of structures $\mathbf{2 4}$ and $\mathbf{2 5}$ do not line up with those of the subsequent structures in the plan with respect to the 2,2-dimethyl-[1,3]-dioxolane group. Note how the hashed wedges change to dark wedges, which implies that a mental flip of the structure is required to read the diagram. A number of intermediate structures are not shown due to space limitations in the original representation which interrupts the flow of reading the scheme and hence following what is going on in the synthesis. By contrast, Scheme 21 redisplays the scheme showing intermediate products for all steps and maintaining a consistent structural aspect for all structures with respect to that of the final product. We can readily see that the plan consists of 9 individual steps in a linear sequence. The first step is an oxidative cleavage reaction producing an aldehyde group. The second step is an addition reaction onto that aldehyde forming a $\mathrm{C}=\mathrm{C}$ bond. The third step is a reductive lactone ring opening reaction leading to a second aldehyde group. The fourth step is an imination reaction onto this aldehyde. The fifth step is a basecatalyzed elimination reaction producing an alkyne. The sixth step is a Heck coupling reaction. The seventh step is a tandem photochemical addition-cyclization reaction. The eighth step is a second cyclization in which a $\delta$-lactam is formed and the thiophenoxide group is eliminated. Finally, the ninth step is an acid-catalyzed deprotection leading to the triol product. Based on the highlighted target bonds, the overall ring mapping for the [4.4.0] fused bicyclic ring motif in the product structure is $\left[(3+1+1+1)_{\mathrm{A}}+(5+1)_{\mathrm{B}}\right]$ where the A subscript refers to the $\delta$-lactam ring and the B subscript refers to the cyclohexane ring. The ring construction strategy based on the synthesis scheme can be described as $\stackrel{[6+0]}{\longrightarrow} B_{7} \stackrel{[6+0]}{\longrightarrow} A_{8}$, where ring $\mathrm{B}$ is made by a $[6+0]$ cyclization in step 7 and ring $\mathrm{A}$ is made by another [6+0] cyclization in step 8. As in the antipyrine case, we can pinpoint all of the atoms from the reagents that contribute to the product structure. Any reagents that did not contribute any atoms are termed sacrificial reagents. In this case, the following sacrificial reagents were employed throughout the synthesis: sodium 
periodiate (step 1), L-selectride (step 3), $n$-butyllithium (step 5), thiophenol (step 7), and water (step 9).

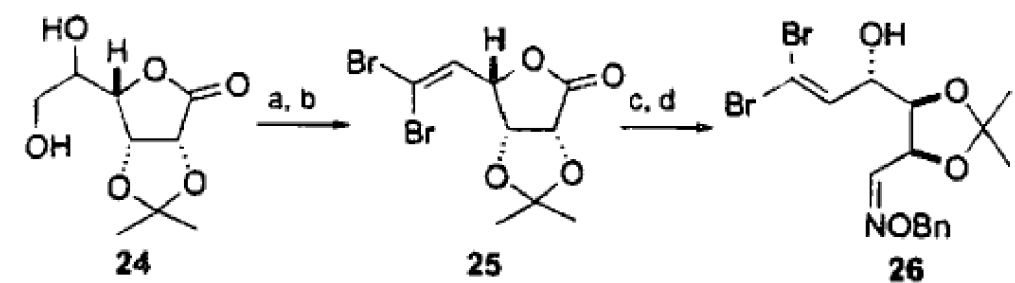

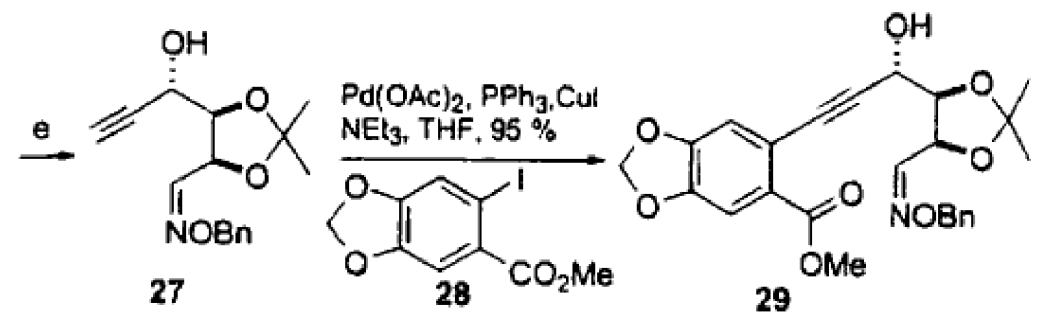

1. $\mathrm{PhSH}, \mathrm{hv}$, tol. $27{ }^{\circ} \mathrm{C} .90 \%$

2. $\mathrm{Smi}_{2}$. THF. $86 \%$

3. TFA, $90 \%$

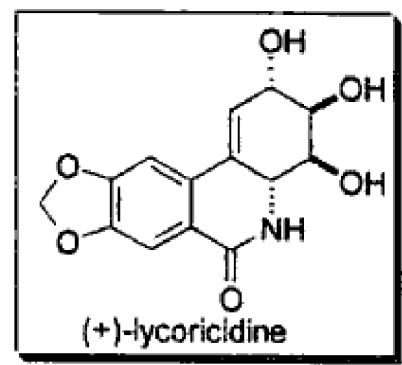

“Key: (a) $\mathrm{NaIO}_{4}, \mathrm{CH}_{2} \mathrm{Cl}_{2}$. (b) $\mathrm{CBr}_{4}, \mathrm{PPh}_{3}, \mathrm{NEt}_{3}, 80 \%$ over two steps. (c) L-Selectride, $\mathrm{Et}_{2} \mathrm{O},-78{ }^{\circ} \mathrm{C}$. (d) $\mathrm{HCl} \cdot \mathrm{H}_{2} \mathrm{NOBn}$, pyridine, $90 \%$ over two steps. (e) ${ }^{n} \mathrm{BuLi}, \mathrm{Et}_{2} \mathrm{O},-90^{\circ} \mathrm{C}, 93 \%$.

Scheme 20. Synthesis plan for (+)-lycoridicine as drawn by the authors [33]. 


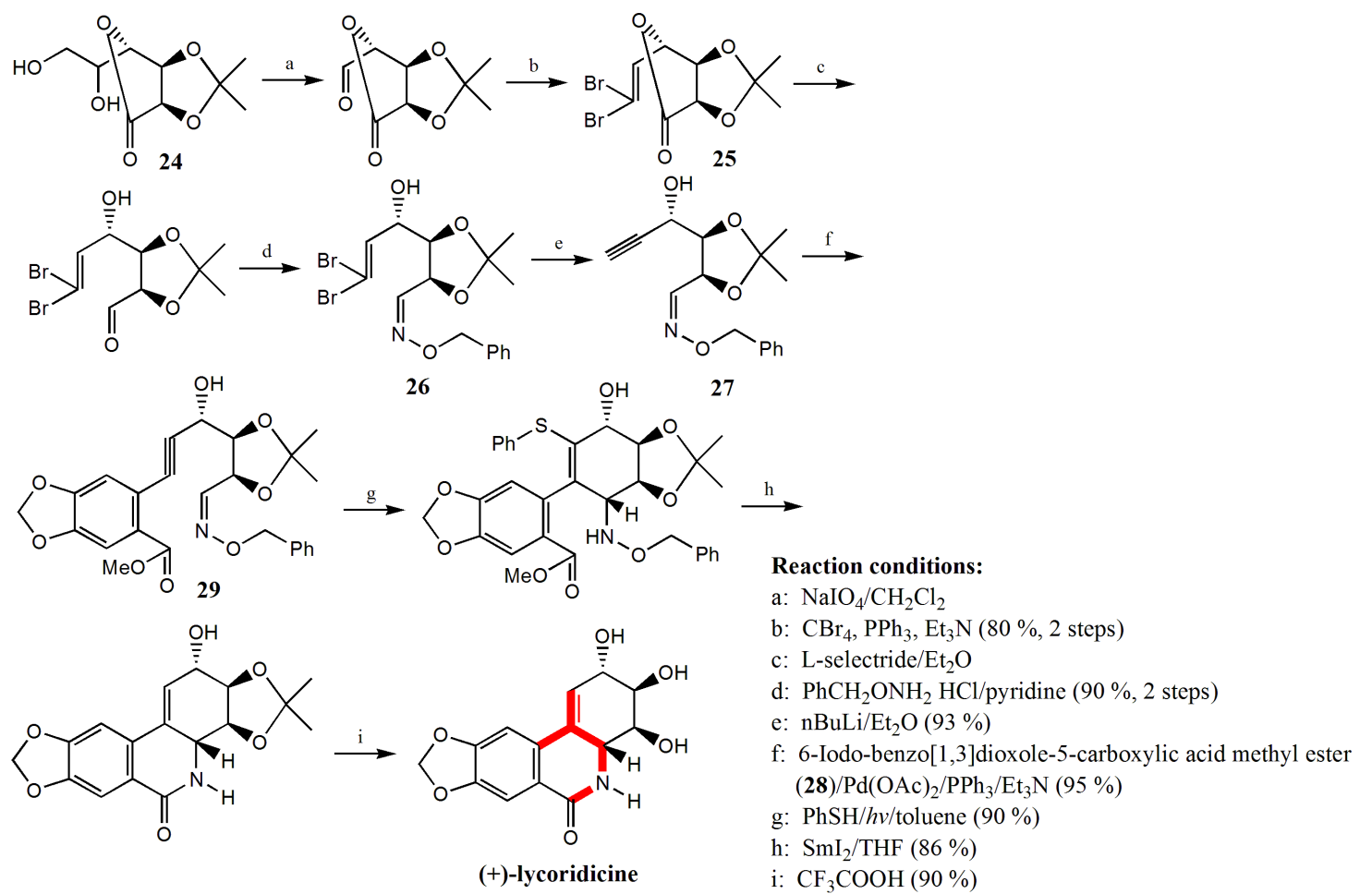

Scheme 21. Synthesis plan for (+)-lycoridicine drawn using the principle of conservation of structural aspect.

\section{Concluding Remarks}

In this work we have demonstrated the application of the principle of conservation of structural aspect as a useful pedagogical guide for the display of individual reactions and synthesis plans. Our experience has shown that student understanding and engagement in the study of introductory organic chemistry improves dramatically as a consequence of applying this principle and also by training students' visualization aptitude through preliminary drawing and mental reflection and rotation exercises. When students are exposed to reactions for the first time it is paramount that effective clarity of visual communication is exercised. We suggest that instructors and textbook writers take the time to present all of their course materials with these techniques in mind in order to bring about better pedagogical outcomes and hopefully reverse the negativity surrounding organic chemistry instruction. In particular, we point out that the common practice of displaying reactions on tests and exams with embedded visual trickery without training 
students in advance of visual techniques to "unwind" them has significant negative pedagogical consequences. We have successfully used TOC graphics in journal articles as an effective springboard for training students to write out chemical equations and synthesis plans that conform to the conservation of structural aspect principle.

\section{Supporting Information}

ChemDraw reflection and rotation exercises; TOC graphics exercises with answers taken from Organic Letters; and synthesis plan exercises.

\section{Acknowledgements}

Parts of this work were presented at the $23^{\text {rd }}$ IUPAC International Conference on Chemical Education (ICCE) held on July 13-18, 2014 at Toronto, Canada.

\section{References}

[1] Hoffmann, R.; Laszlo, P. Representation in chemistry. Angew. Chem. Int. Ed. 1999, 30, 1-16.

[2] Wu, H.K.; Shah, P. Exploring visuospatial thinking in chemistry learning. Science Educ. 2004, 88, 465-492.

[3] Harle, M.; Towns, M. A review of spatial ability literature, its connection to chemistry, and implications for instruction. J. Chem. Educ. 2011, 88, 351-360.

[4] Habraken, C.L. Perceptions of chemistry: why is the common perception of chemistry, the most visual of sciences, so distorted? J. Sci. Educ. Techn. 1996, 5, 193201.

[5] Luisi, P.L.; Thomas, R.M. The pictographic molecular paradigm: pictorial communication in the chemical and biological sciences. Naturwissenschaften 1990, 77, 67-74. 
[6] Goodwin, W. Visual representations in science. Phil. Sci. 2009, 76, 372-390.

[7] Stieff, M. When is a molecule three-dimensional? A task specific role for imagistic reasoning in advanced chemistry. Science Educ. 2011, 95, 310-336.

[8] Stieff, M.; Raje, S. Expert algorithmic and imagistic problem solving strategies in advanced chemistry. Spatial Cogn. Comput. 2010, 10, 53-81.

[9] Tuckey, H.; Selvaratnam, M. Studies involving three-dimensional visualisation skills in chemistry: a review. Studies Sci. Educ. 1993, 21, 99-121.

[10] Halford, B. Overwhelmed by Orgo: The Crisis Facing Organic Chemistry Educators is Not a New One, They Say. Chem. Eng. News 2016, 94(13), 24-25.

[11] Ferguson, R.; Bodner, G.M. Making sense of the arrow-pushing formalism among chemistry majors enrolled in organic chemistry. Chem. Educ. Res. Practice 2008, 9, 102113.

[12] Flynn, A.B.; Featherstone, R.B. Language of mechanisms: exam analysis reveals students' strengths, strategies, and errors when using the electron-pushing formalism (curved arrows) in new reactions. Chem. Educ. Res. Practice 2017, 18, 64-91.

[13] Bhattacharyya, G.; Bodner, G.M. "It Gets Me to the Product": How Students Propose Organic Mechanisms. J. Chem. Educ. 2005, 82, 1402-1407.

[14] Bhattacharyya, G. From Source to Sink: Mechanistic Reasoning Using the Electron-Pushing Formalism. J. Chem. Educ. 2013, 90, 1282-1289.

[15] Bodé, N.E.; Flynn, A.B. Strategies of Successful Synthesis Solutions: Mapping, Mechanisms, and More. J. Chem. Educ. 2016, 93, 593-604. 
[16] Galloway, K.R.; Leung, M.W.; Flynn, A.B. A Comparison of How Undergraduates, Graduate Students, and Professors Organize Organic Chemistry Reactions. J. Chem. Educ. 2018, 95, 355-365.

[17] Shepard, R.N.; Metzler, J. Mental Rotation of Three-dimensional Objects. Science 1971, 171, 701-703.

[18] Li, J.J. Name Reactions - a collection of detailed mechanisms and synthetic applications, $5^{\text {th }}$ ed., Springer: Heidelberg, 2014.

[19] Hassner, A.; Namboothiri, I. Organic Syntheses Based on Name Reactions - a practical guide to 750 transformations, $3^{\text {rd }}$ ed., Elsevier: Amsterdam, 2012.

[20] Kidwai, M.; Mohan, R. Green chemistry: an innovative technology. Foundations of Chemistry 2005, 7, 269-287.

[21] Hultin, P.G. The What, How and Why of Problem Solving in Organic Chemistry... and elsewhere!, University of Manitoba, 2004 http://home.cc.umanitoba.ca/ hultin/chem2220/Support/WhatHowWhy.pdf

[22] Solomons, T.W.G. Organic Chemistry, $6^{\text {th }}$ ed., Wiley: New York, 1996, p. 530.

[23] Meinwald, J. The Acid-catalyzed Rearrangement of Cinenic Acid. J. Org. Chem. $1955,77,1617-1620$.

[24] Meinwald, J.; Cornwall, C.C. The Acid-catalyzed Rearrangement of Cinenic Acid. II. Geronic Acid from 6-Hydroxy-2,Z,6-trimethylcyclohexanone. J. Org. Chem. 1955, 77, 5991-5992.

[25] Meinwald, J.; Hwang, H.C. The Acid-catalyzed Rearrangement of Cinenic Acid. III. Structure and Synthesis of the Lactonic Product. J. Org. Chem. 1957, 79, 2910-2912. 
[26] Meinwald, J.; Ouderkirk, J.T. The Acid-catalyzed Rearrangement of Cinenic Acid. IV. Synthesis and Rearrangement of 6-Carboxy-6-ethyl-2,2dimethyltetrahydropyran. J. Org. Chem. 1960, 82, 480-483.

[27] Meinwald, J.; Hwang, H.C.; Christman, D.; Wolf, A.P. The Acid-catalyzed Rearrangement of Cinenic Acid. V. Evidence for a Decarbonylation-Recarbonylation Mechanism. J. Org. Chem. 1960, 82, 483-486.

[28] Wang, L.; Liu, Q.B.; Wang, D.S.; Li, X.; Han, X.W.; Xiao, W.J.; Zhou, Y.G. Tandem Ring-Opening/Closing Reactions of N-Ts Aziridines and Aryl Propargyl Alcohols Promoted by t-BuOK. Org. Lett. 2009, 11, 1119-1122.

[29] Zheng, Z.; Alper, H. Palladium-Catalyzed Cyclocarbonylation-Decarboxylation of Diethyl(2-iodoaryl)malonates with Vinyl Ketones Affording Functionalized Enolic 2-Acyl3,4-dihydronaphthalenones. Org. Lett. 2009, 11, 3278-3281.

[30] Déry, M.; Lefebvre, L.P.D.; Aissa, K.; Spino, C. $N$-Heteropolycyclic Compounds from the Formal Intramolecular $(4+1)$-Cycloaddition of Chromium Aminocarbenes. Org. Lett. 2013, 15, 5456-5459.

[31] Cheung, L.L.W.; Yudin, A.K. Synthesis of Aminocyclobutanes through Ring Expansion of N-Vinyl- $\beta$-Lactams. Org. Lett. 2009, 11, 1281-1284.

[32] Furniss, B.S.; Hannaford, A.J.; Rogers, V.; Smith, P.W.G.; Tatchell, A.R. Vogel's Textbook of Practical Organic Chemistry, Longman: London, 1978, p. 540, 727, 882.

[33] Keck, G. E.; Wager, T. T.; Rodriquez, J. F. D. Total syntheses of (-)-lycoridicine, (+)-lycoridicine, and (+)-narciclasine via 6-exo-cyclizations of substituted vinyl radicals with oxime ethers. J. Am. Chem. Soc. 1999, 121, 5176-5190. 
TOC graphic

\section{Principle of Conservation of Structural Aspect}

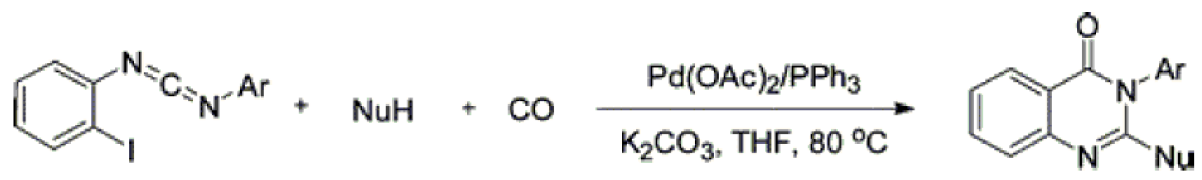

versus
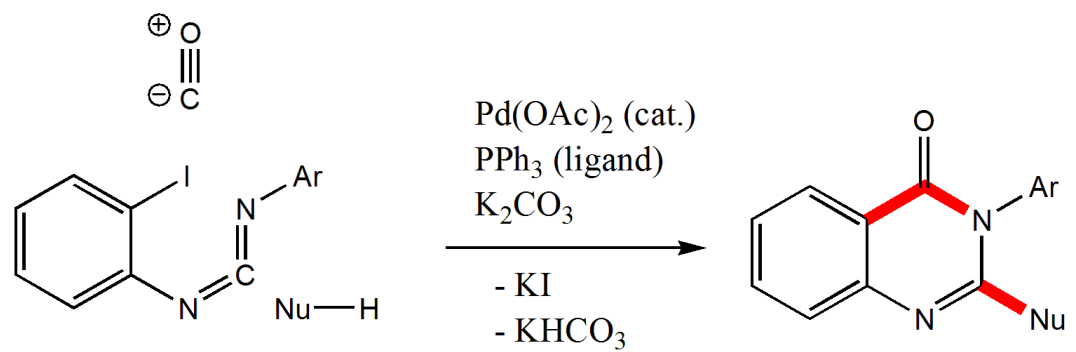
The Principle of Conservation of Structural Aspect: Facilitating Visual Communication and Learning in Organic Chemistry Instruction

John Andraos

CareerChem

504-1129 Don Mills Road

Toronto, ON M3B 2W4

Canada

c1000@careerchem.com

\section{Supporting Information}

Table of Contents:

I. ChemDraw Ultra 7.0 mental rotation exercises pg 2

II. ChemDraw Ultra 7.0 mental reflection exercises pg 4

III. Journal TOC graphics exercises taken from Organic Letters (2009-2013) pg 7

IV. Ring construction and mapping exercises $\quad$ pg 64

$\begin{array}{lll}\text { V. Synthesis schemes exercises } & \text { pg } 68\end{array}$ 


\section{ChemDraw Ultra 7.0 mental rotation exercises}

Exercise \#1

Rotate each image by $+90,-90,+180$, and -180 degrees.
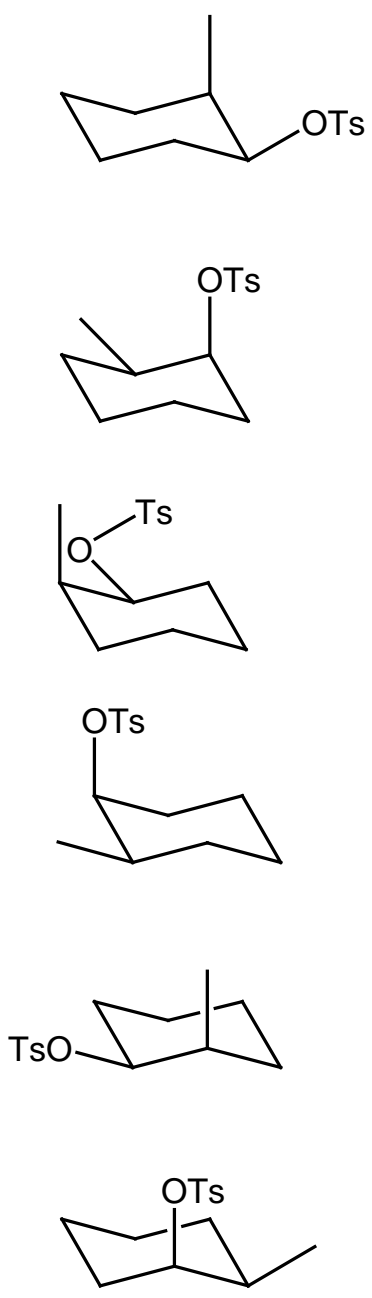

Exercise \#2

Rotate each image by $+90,-90,+180$, and -180 degrees 
<smiles>COc1ccc(C)cc1F</smiles><smiles>COc1cc(C)ccc1C</smiles><smiles>COc1ccc(C)cc1</smiles><smiles>Cc1cccc(F)c1</smiles><smiles>COc1cccc(F)c1C</smiles>

\section{Exercise \#3}

Rotate each image by $+90,-90,+180$, and -180 degrees . 
<smiles>C[C@H](O)C(O)[C@H](C)O</smiles><smiles>C[C@H](O)C(O)[C@H](C)O</smiles><smiles>C[C@H](O)C(O)[C@H](C)O</smiles><smiles>C[C@H](O)C(O)[C@H](C)O</smiles><smiles>C[C@H](O)C(O)[C@H](C)O</smiles>

\section{ChemDraw Ultra 7.0 mental reflection exercises}

\section{Exercise \#1}

Draw the mirror images of the following structures: 

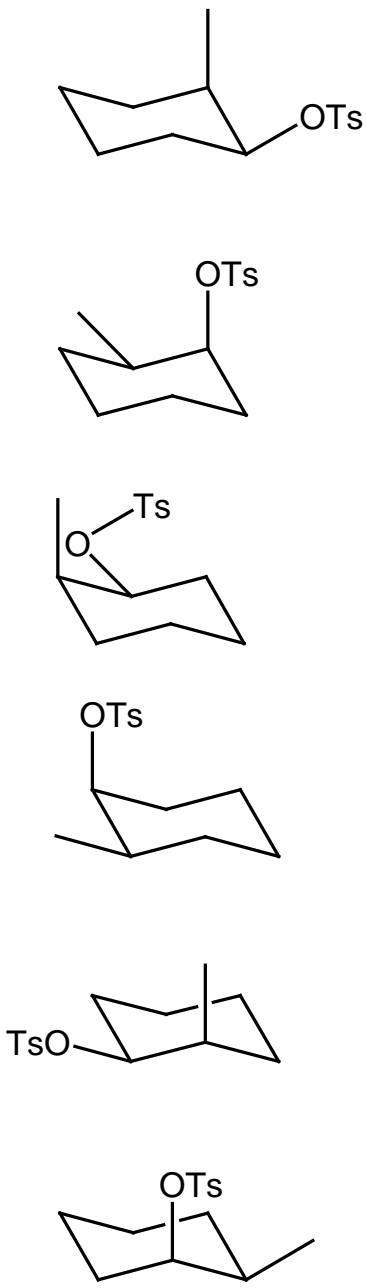

Exercise \#2

Draw the mirror images of the following structures: 
<smiles>COc1ccc(C)cc1F</smiles><smiles>COc1cc(C)ccc1C</smiles><smiles>COc1ccc(C)cc1</smiles><smiles>Cc1cccc(F)c1</smiles><smiles>COc1cccc(F)c1C</smiles>

\section{Exercise \#3}

Draw the mirror images of the following structures: 
<smiles>C[C@H](O)C(O)[C@H](C)O</smiles><smiles>C[C@H](O)C(O)[C@H](C)O</smiles><smiles>C[C@H](O)C(O)[C@H](C)O</smiles><smiles>C[C@H](O)C(O)[C@H](C)O</smiles><smiles>C[C@H](O)C(O)[C@H](C)O</smiles>

III. Journal TOC graphics exercises taken from Organic Letters (2009-2013)

Examine each TOC graphic shown below. Indicate if the TOC graphic obeys the principle of conservation of structural aspect. If it does not, then re-draw the reaction using the principle of conservation of structural aspect and indicate the target bonds made in each reaction using bolded lines. Comment on colour-coding strategies if they are used.

\section{Exercise \#1}

Liang Wang, Qi-Bin Liu, Duo-Sheng Wang, Xin Li, Xiu-Wen Han, Wen-Jing Xiao and Yong-Gui Zhou. Tandem Ring-Opening/Closing Reactions of N-Ts Aziridines and Aryl Propargyl Alcohols Promoted by t-BuOK. Org. Lett. 2009, 11, 1119-1122. 


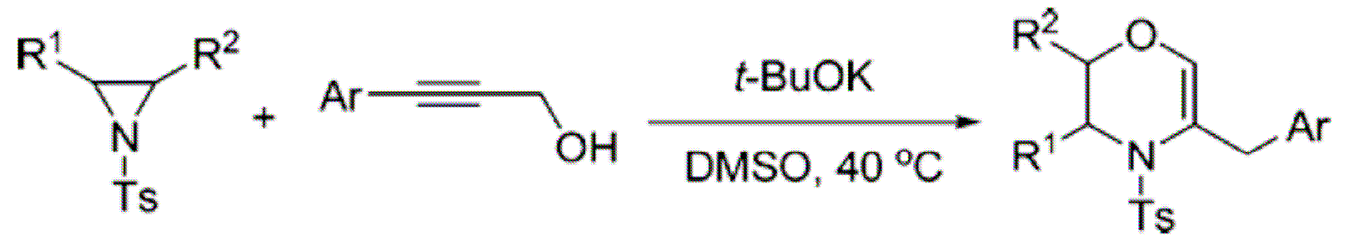

Exercise \#2

Lawrence L. W. Cheung and Andrei K. Yudin. Synthesis of Aminocyclobutanes through Ring Expansion of N-Vinyl- $\beta$-Lactams. Org. Lett. 2009, 11, 1281-1284.
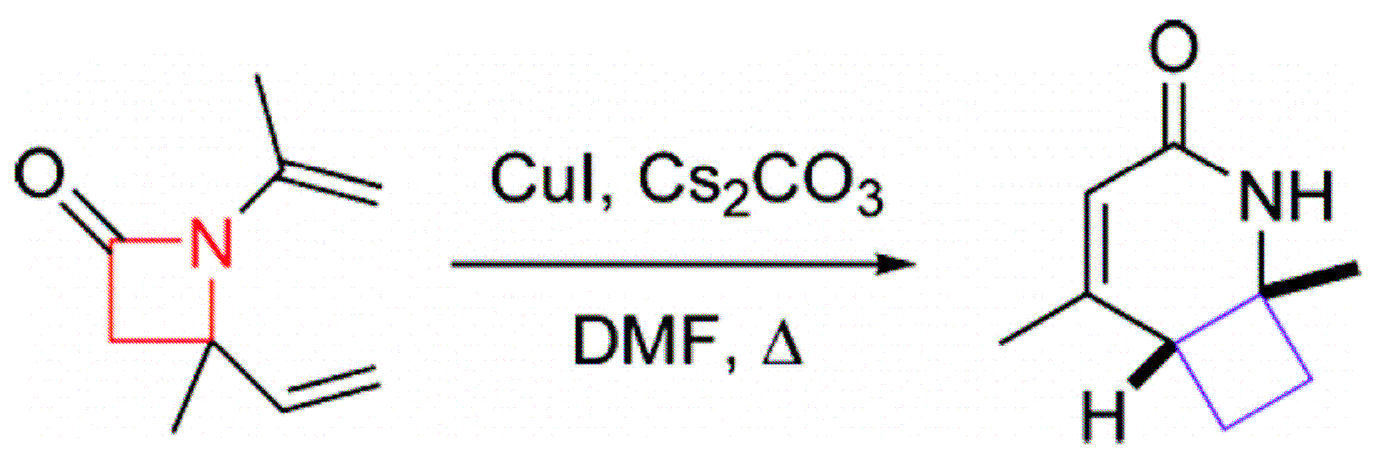

\section{Exercise \#3}

Yingdong Lu and Bruce A. Arndtsen. A Direct Phosphine-Mediated Synthesis of Pyrroles from Acid Chlorides and $\alpha, \beta$-Unsaturated Imines. Org. Lett. 2009, 11, 1369-1372.<smiles>[R]C=C([R])C([R])=N[R]</smiles><smiles>[R5]C(=O)Cl</smiles><smiles>[R]c1c([R])c([R7])n([R])c1[R]</smiles>

\section{Exercise \#4}

Min-Tsang Hsieh, Ho-Hsuan Chou, Hsing-Jang Liu, Huang-Min Wu, Tai Wei Ly,

Yen-Ku Wu and Kak-Shan Shia. Polyene Cyclization Promoted by the Cross-Conjugated $\alpha$-Carbalkoxy Cyclohexenone System. An Unusual 1,2-Hydride Shift under Lewis Acid Catalysis. Org. Lett. 2009, 11, 1673-1675.

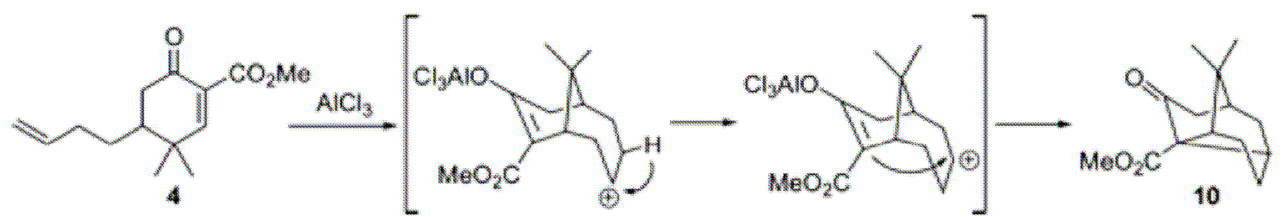

Exercise \#5

Zhaoyan Zheng and Howard Alper. Palladium-Catalyzed Cyclocarbonylation-

Decarboxylation of Diethyl(2-iodoaryl)malonates with Vinyl Ketones Affording

Functionalized Enolic 2-Acyl-3,4-dihydronaphthalenones. Org. Lett. 2009, 11, 3278-3281. 


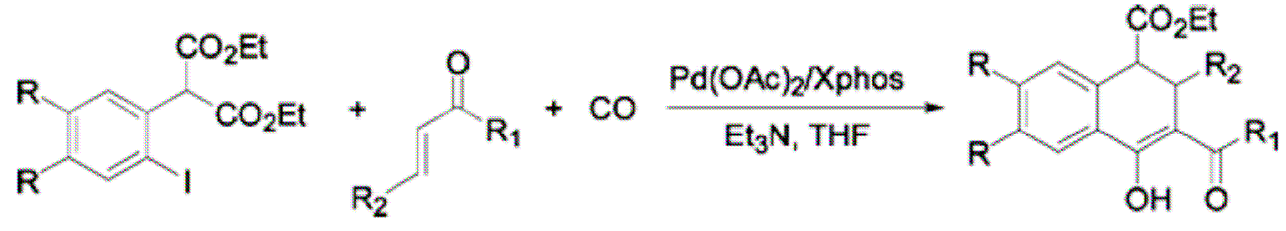

\section{Exercise \#6}

Andrew G. Lohse and Richard P. Hsung. Thermal Intramolecular [4 + 2] Cycloadditions of Allenamides: A Stereoselective Tandem Propargyl Amide Isomerization-Cycloaddition. Org. Lett. 2009, 11, 3430-3433.

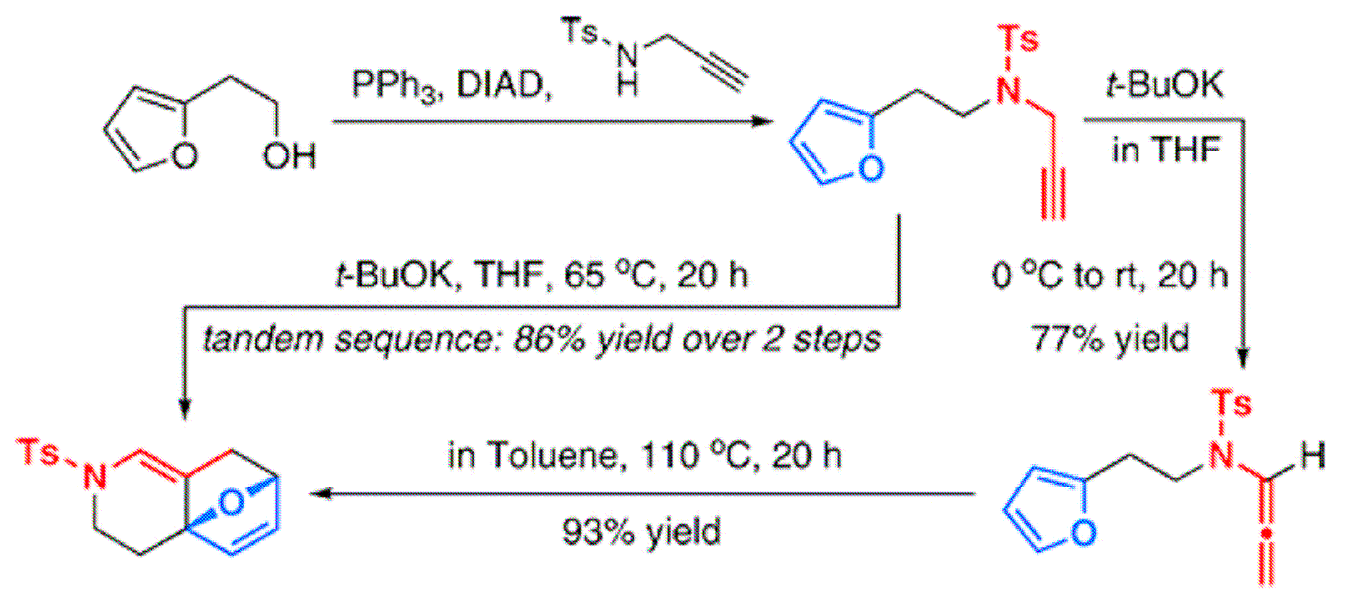

\section{Exercise \#7}

Dwayne A. Dias and Michael A. Kerr. Domino Synthesis of Bridged Bicyclic Tetrahydro1,2-oxazines: Access to Stereodefined 4-Aminocyclohexanols. Org. Lett. 2009, 11, 36943697.

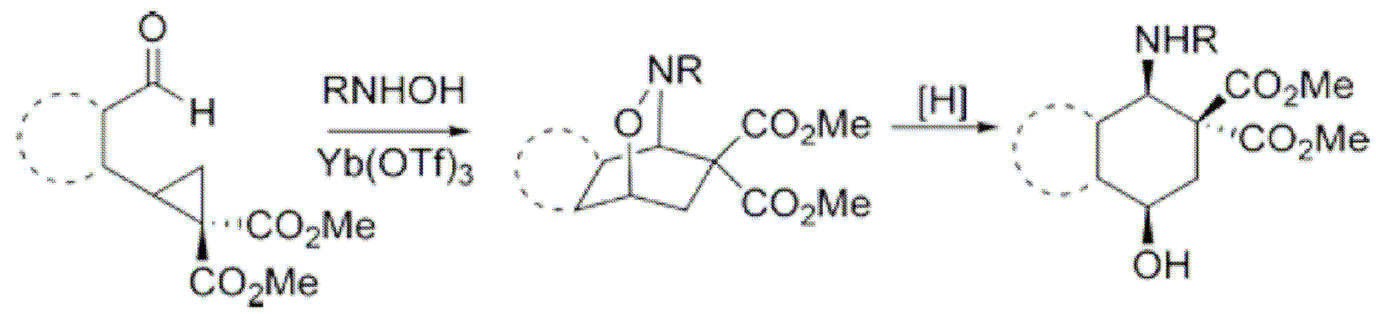

\section{Exercise \#8}

Terry P. Lebold, Andrew B. Leduc and Michael A. Kerr. Zn(II)-Catalyzed Synthesis of Piperidines from Propargyl Amines and Cyclopropanes. Org. Lett. 2009, 11, 3770-3772. 
<smiles>[R]C(C#C)NCc1ccccc1</smiles>
$\mathrm{Zn}\left(\mathrm{NTf}_{2}\right)_{2}(10 \mathrm{~mol} \%)$ refluxing benzene

$59-99 \%$<smiles>[R]C1CC(C(C)=O)(C(C)=O)C(=C)C([R])N1Cc1ccccc1</smiles>

15 examples

Exercise \#9

Suqing Zheng and Xiyan Lu. Phosphine-Catalyzed [4 + 3] Annulation for the Synthesis of Highly Functionalized Bicyclo[3.2.2]nonadienes. Org. Lett. 2009, 11, 3978-3981.

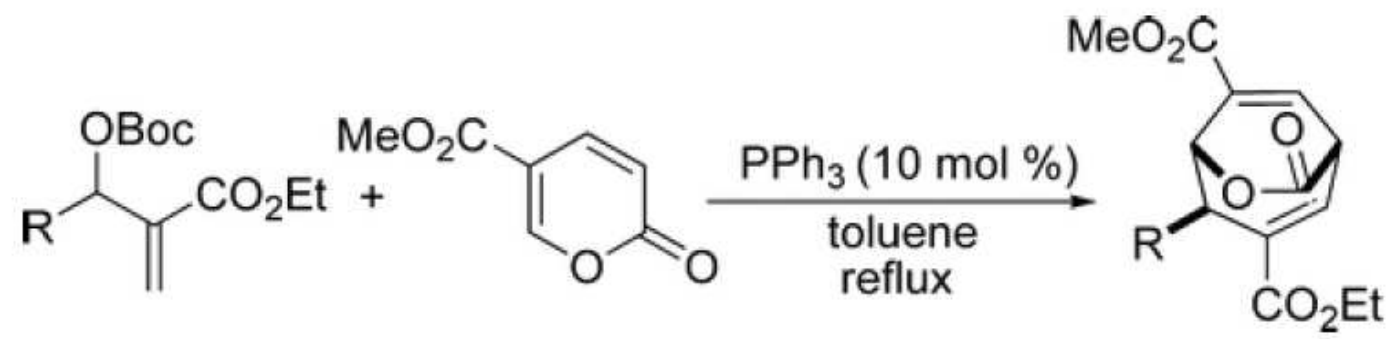

Exercise \#10

Weibing Liu, Huanfeng Jiang and Liangbin Huang. One-Pot Silver-Catalyzed and PIDA-Mediated Sequential Reactions: Synthesis of Polysubstituted Pyrroles Directly from Alkynoates and Amines. Org. Lett. 2010, 12, 312-315.

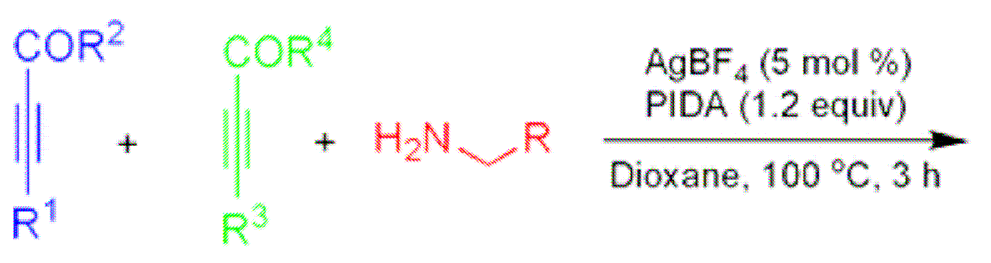<smiles>[R]Cn1c(C([R])=[R])c([R])c([R])c1C(=O)O</smiles>

Exercise \#11

Zhenqiu Guo, Mei Cai, Jun Jiang, Liping Yang and Wenhao Hu. Rh2(OAc)4-AgOTf Cooperative Catalysis in Cyclization/Three-Component Reactions for Concise Synthesis of 1,2-Dihydroisoquinolines. Org. Lett. 2010, 12, 652-655.<smiles>[R20]OC(=O)[C@H]([R])C</smiles>

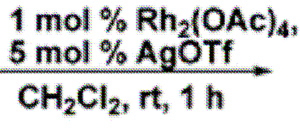<smiles>[R]OC(=O)C([R])C1c2ccccc2C=C(c2ccccc2)N1[R]</smiles> 
Exercise \#12

Siyu Ye and Zhi-Xiang Yu. Gold(I)-Catalyzed Ring Expansions of Unactivated Alkynylcyclopropanes to $(E)$-2-Alkylidenecyclobutanamines in the Presence of Sulfonamides. Org. Lett. 2010, 12, 804-807.

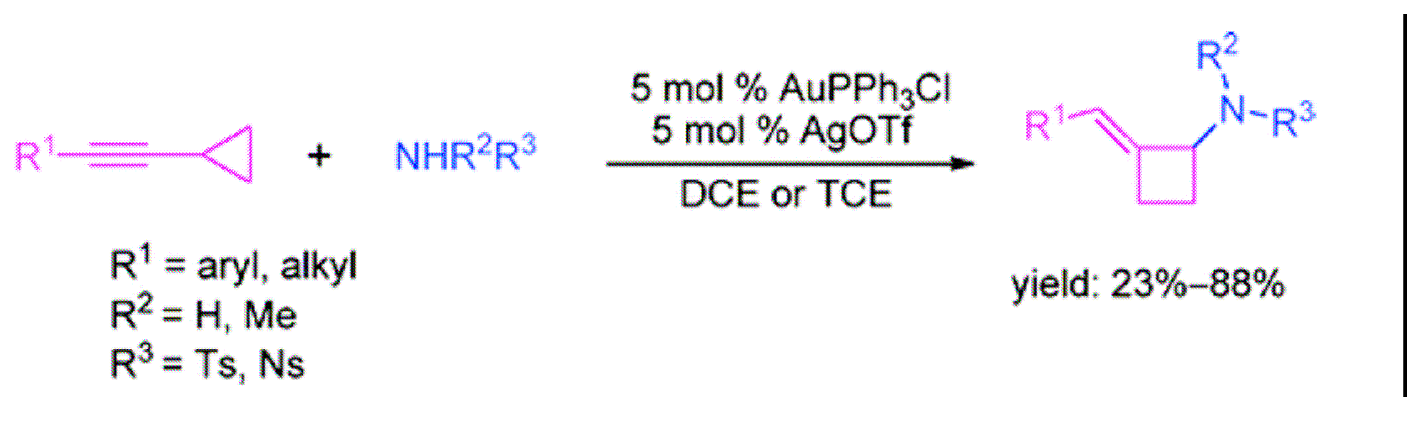

\section{Exercise \#13}

Franklin A. Davis and Ram Edupuganti. Asymmetric Synthesis of Substituted Homotropinones from $N$-Sulfinyl $\beta$-Amino Ketone Ketals. (-)-Euphococcinine and (-)Adaline. Org. Lett. 2010, 12, 848-851.<smiles>CC(=O)CC(CCCC1(C)OCCO1)NS(=O)O</smiles>

$\left(S_{S}, 4 S\right) \cdot(+)$

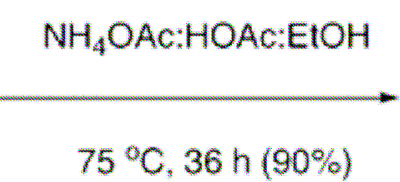

$75^{\circ} \mathrm{C}, 36 \mathrm{~h}(90 \%)$

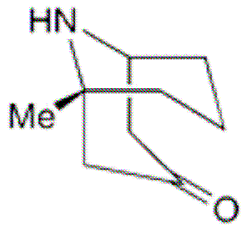

(-)-euphococcinine

Exercise \#14

Shirong Lu, Tienan Jin, Ming Bao and Yoshinori Yamamoto. Palladium-Catalyzed Three-Component [3 + 2] Cycloaddition of Propargyl Trifluoroacetates, Ethylidene Malononitriles, and Allyltributylstannane. Org. Lett. 2010, 12, 864-866.
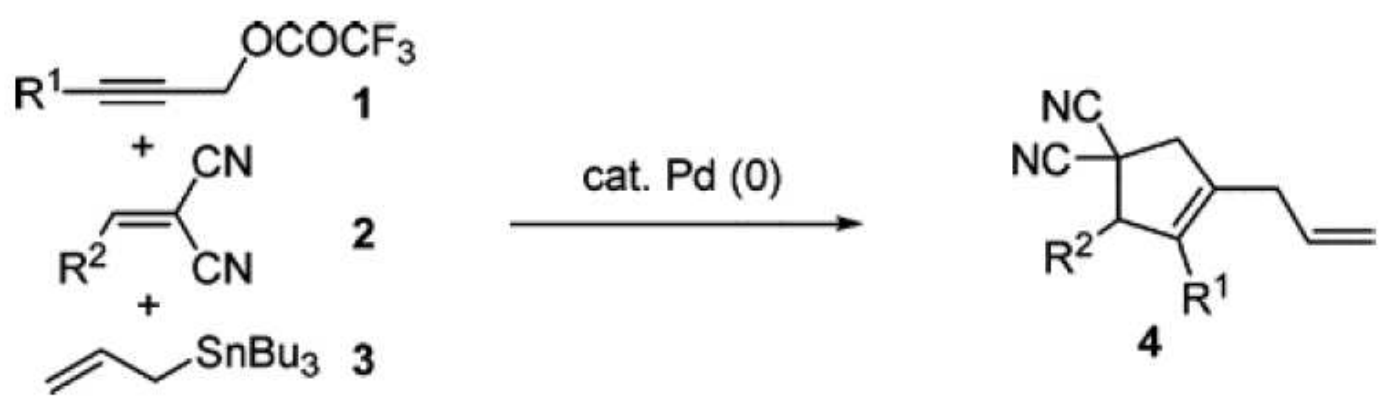

Exercise \#15 
Takanori Matsuda, Sho Kadowaki, Yoshiyuki Yamaguchi and Masahiro Murakami. Ruthenium-Catalyzed trans-Hydrogermylation of Alkynes: Formation of 2,5-Disubstituted Germoles through Double trans-Hydrogermylation of 1,3-Diynes. Org. Lett. 2010, 12, 1056-1058.

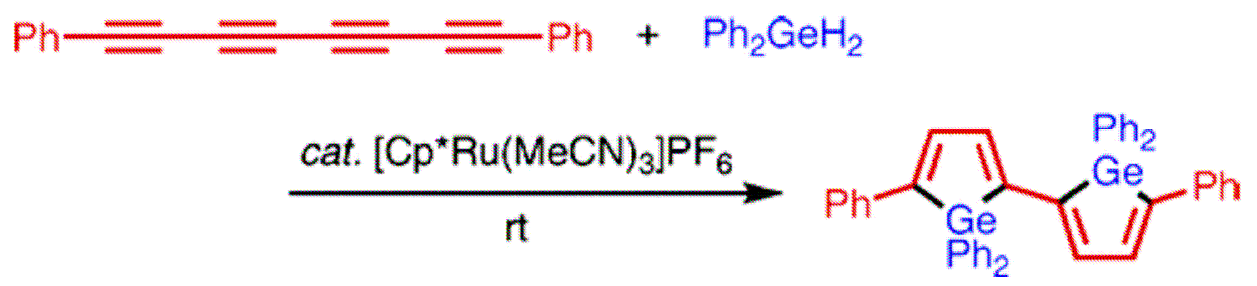

Exercise \#16

Fanlong Zeng and Howard Alper. Tandem Palladium-Catalyzed

Addition/Cyclocarbonylation: An Efficient Synthesis of 2-Heteroquinazolin-4(3H)-ones.

Org. Lett. 2010, 12, 1188-1191.

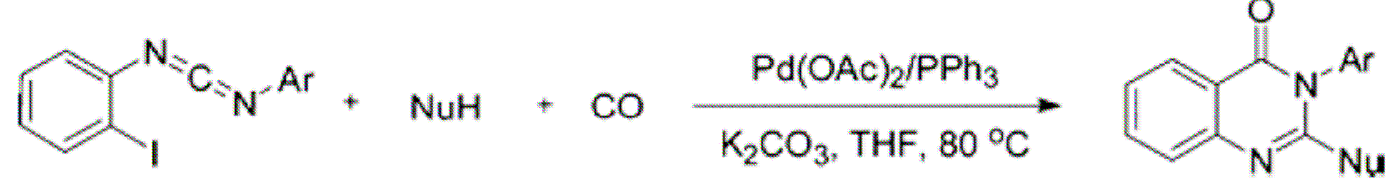

\section{Exercise \#17}

Andrew L. McIver and Alexander Deiters. Tricyclic Alkaloid Core Structures Assembled by a Cyclotrimerization-Coupled Intramolecular Nucleophilic Substitution Reaction. Org. Lett. 2010, 12, 1288-1291.
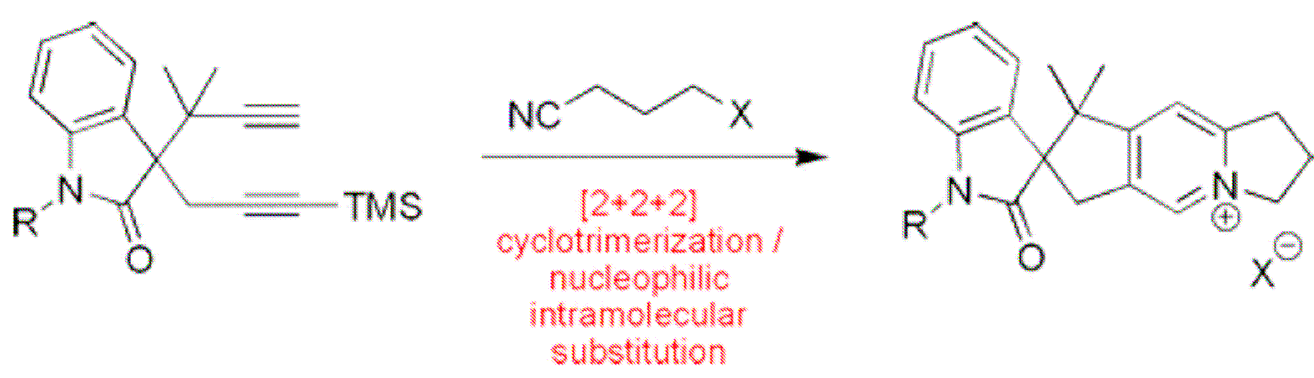

\section{Exercise \#18}

Qian Li, Guo-Jie Jiang, Lei Jiao and Zhi-Xiang Yu. Reaction of $\alpha$-EneVinylcyclopropanes: Type II Intramolecular [5+2] Cycloaddition or [3+2] Cycloaddition? Org. Lett. 2010, 12, 1332-1335. 


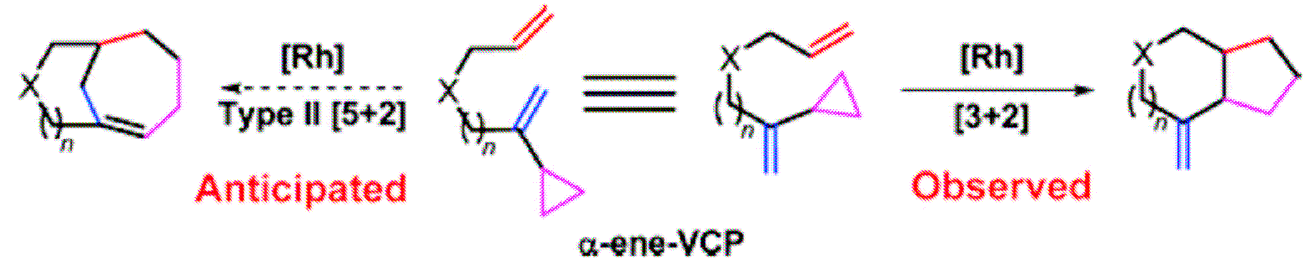

\section{Exercise \#19}

Julien Brioche, Geraldine Masson and Jieping Zhu. Passerini Three-Component Reaction of Alcohols under Catalytic Aerobic Oxidative Conditions.

Org. Lett. 2010, 12, 1432-1435.

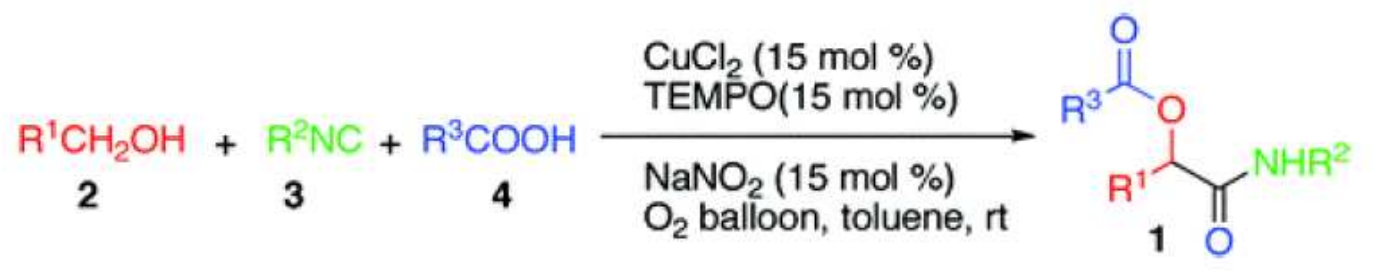

Exercise \#20

Paul A. Wender, Lauren E. Sirois, Rene T. Stemmler and Travis J. Williams. Highly Efficient, Facile, Room Temperature Intermolecular [5 + 2] Cycloadditions Catalyzed by Cationic Rhodium(I): One Step to Cycloheptenes and Their Libraries. Org. Lett. 2010, $12,1604-1607$.
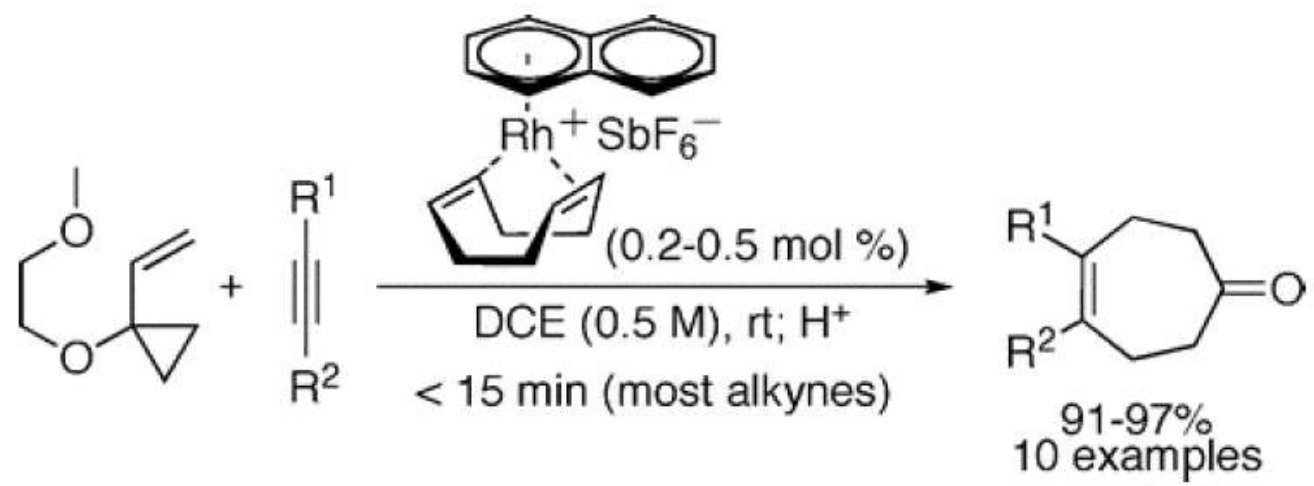

Exercise \#21

Huan Liang and Marco A. Ciufolini. Tandem Phenolic Oxidative Amidation-Intramolecular Diels-Alder Reaction: An Approach to the Himandrine Core. Org. Lett. 2010, 12, 1760-1763. 


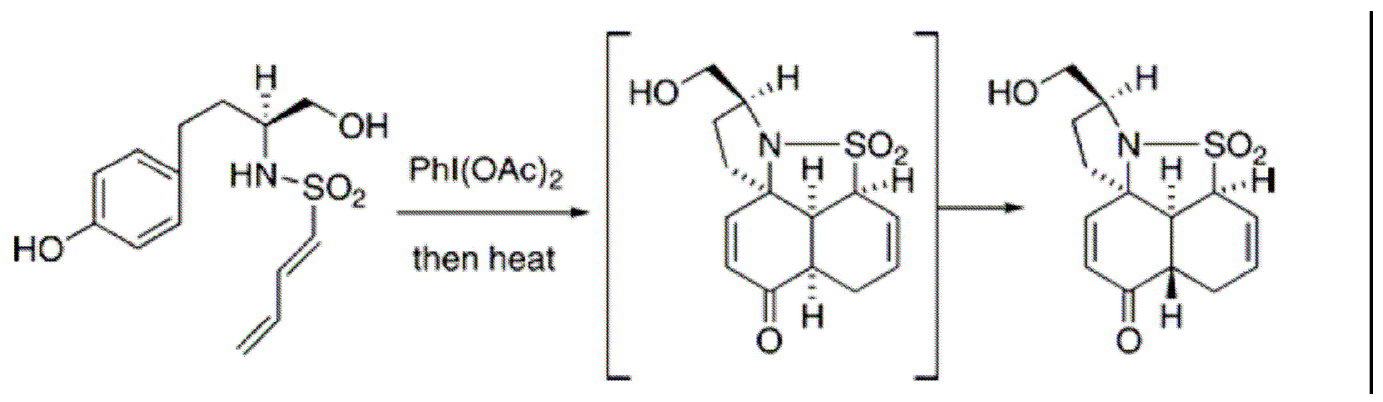

\section{Exercise \#22}

Cheng Zhong, Tao Liao, Odbadrakh Tuguldur and Xiaodong Shi. One-Pot Synthesis of Substituted Di-Hydrofurans from Lewis Base-Catalyzed Three-Component

Condensation. Org. Lett. 2010, 12, 2064-2067.
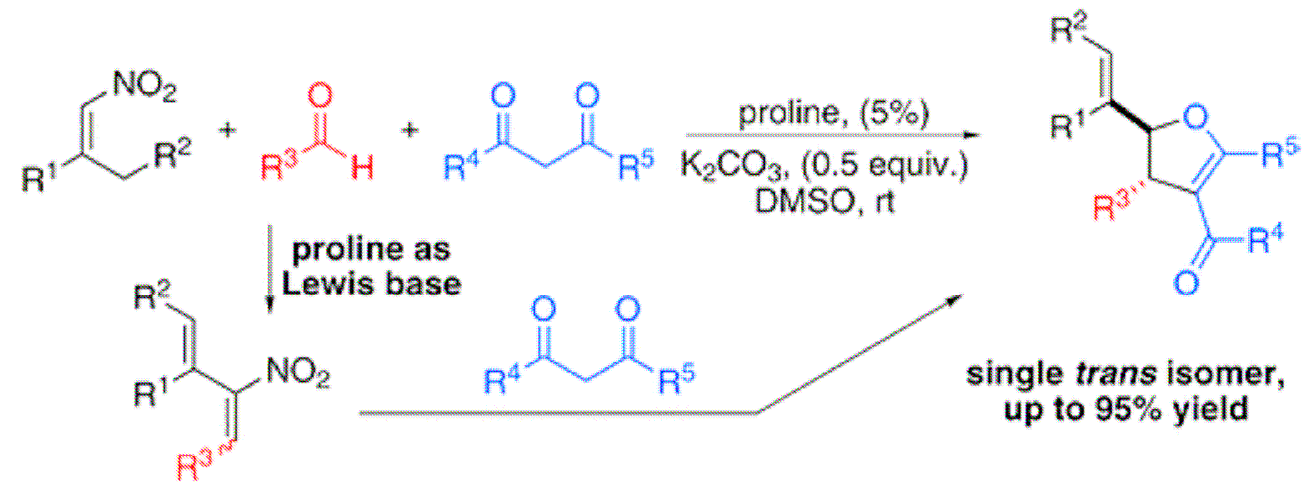

single trans isomer, up to $95 \%$ yield

Exercise \#23

Kadri Kriis, Kerti Ausmees, Tonis Pehk, Margus Lopp and Tonis Kanger. A Novel Diastereoselective Multicomponent Cascade Reaction. Org. Lett. 2010, 12, 2230-2233.

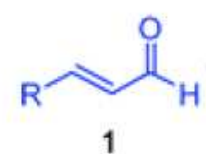<smiles>CCOC(=O)/C=C/CN[Pb]</smiles>

2

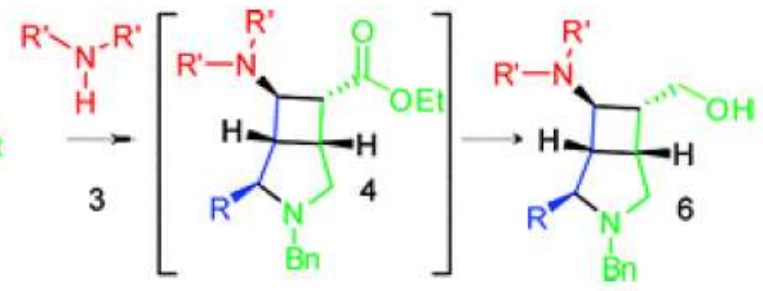

\section{Exercise \#24}

Chao Wang, Zhi-Yong Han, Hong-Wen Luo and Liu-Zhu Gong. Highly

Enantioselective Relay Catalysis in the Three-Component Reaction for Direct

Construction of Structurally Complex Heterocycles. Org. Lett. 2010, 12, 2266-2269. 


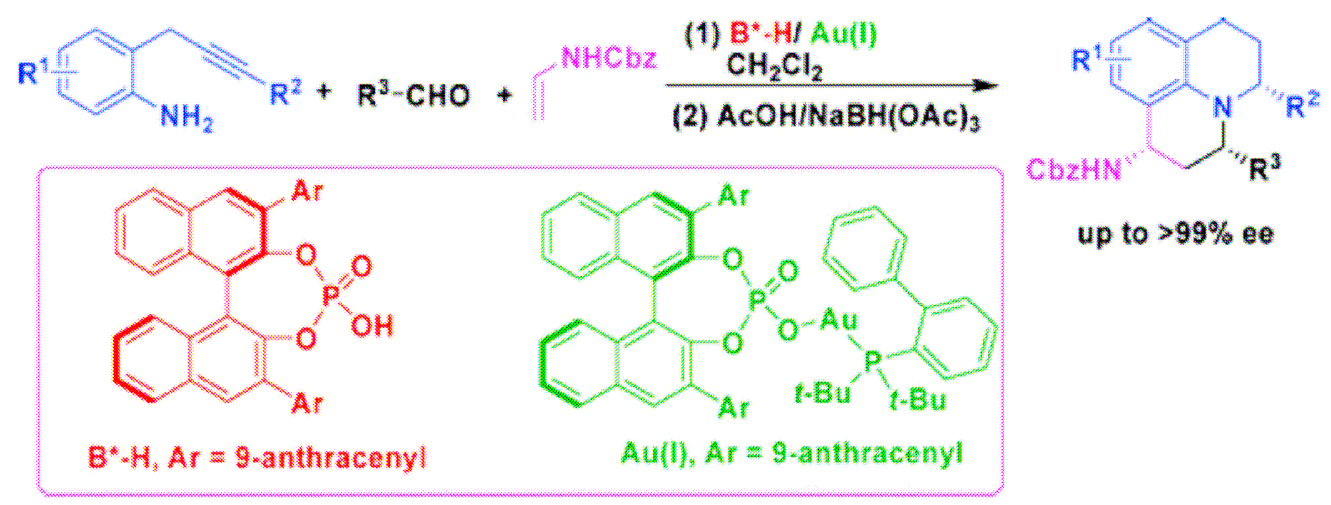

Exercise \#25

Olga P. Pereshivko, Vsevolod A. Peshkov and Erik V. Van der Eycken. Unprecedented $\mathrm{Cu}(\mathrm{I})$-Catalyzed Microwave-Assisted Three-Component Coupling of a Ketone, an Alkyne, and a Primary Amine. Org. Lett. 2010, 12, 2638-2641.
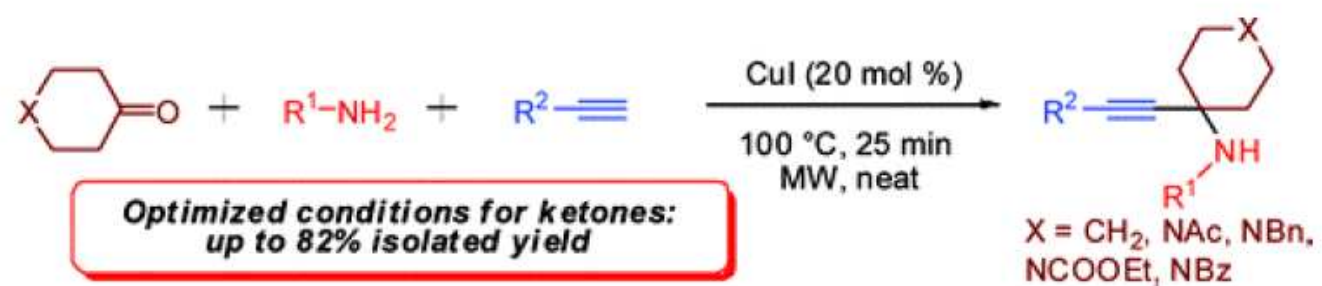

Exercise \#26

Hee-Yoon Lee, Yongsik Jung, Yeokwon Yoon, Byung Gyu Kim and Yeonjoon Kim. Angularly Fused Triquinanes from Linear Substrates through Trimethylenemethane Diyl [2+3] Cycloaddition Reaction. Org. Lett. 2010, 12, 2672-2674.
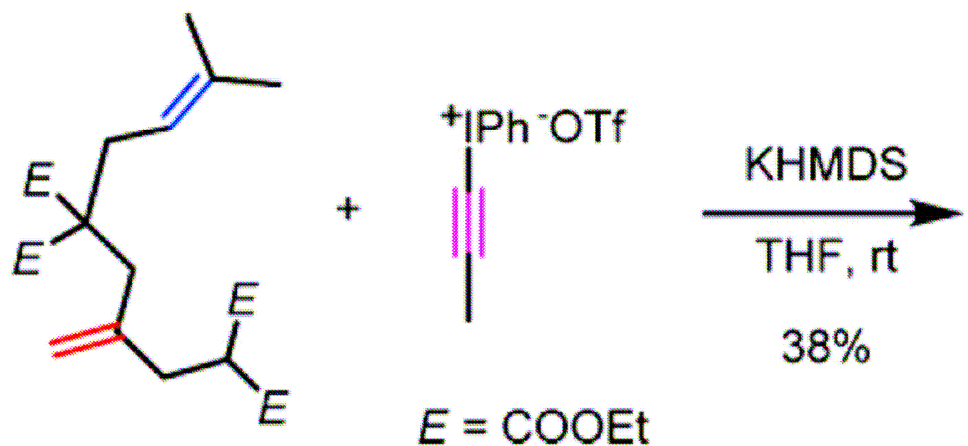

$$
E=\text { COOEt }
$$

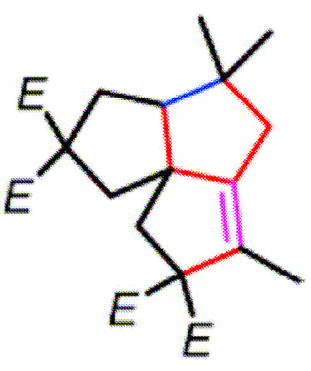

Exercise \#27

Kun Jiang, Zhi-Jun Jia, Xiang Yin, Li Wu and Ying-Chun Chen. Asymmetric Quadruple Aminocatalytic Domino Reactions to Fused Carbocycles Incorporating a Spirooxindole Motif. Org. Lett. 2010, 12, 2766-2769. 


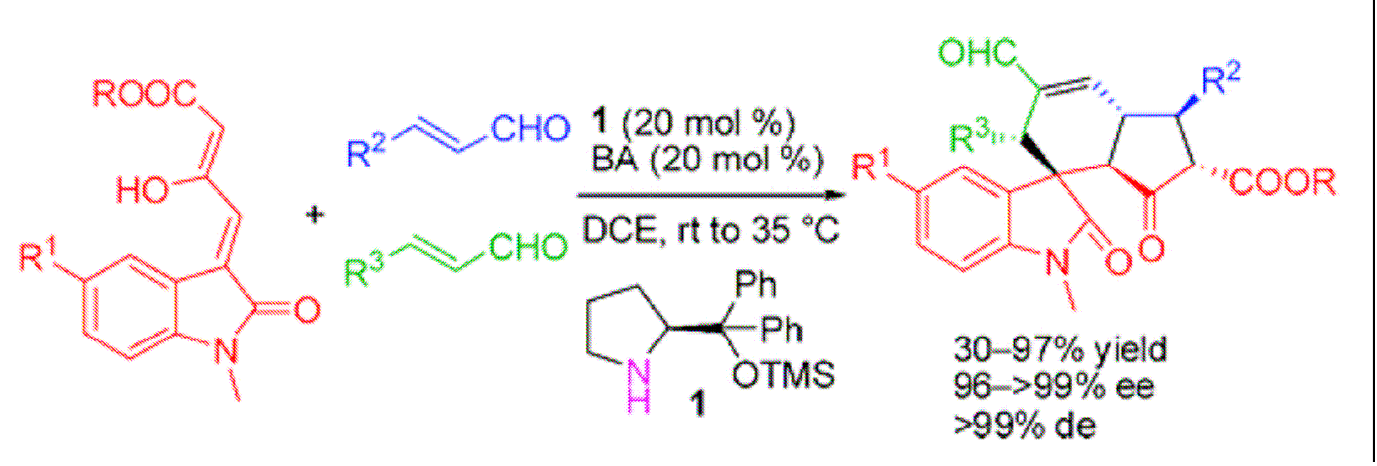

\section{Exercise \#28}

Ali Ramazani and Aram Rezaei. Novel One-Pot, Four-Component Condensation Reaction: An Efficient Approach for the Synthesis of 2,5-Disubstituted 1,3,4-Oxadiazole Derivatives by a Ugi-4CR/aza-Wittig Sequence. Org. Lett. 2010, 12, 2852-2855.

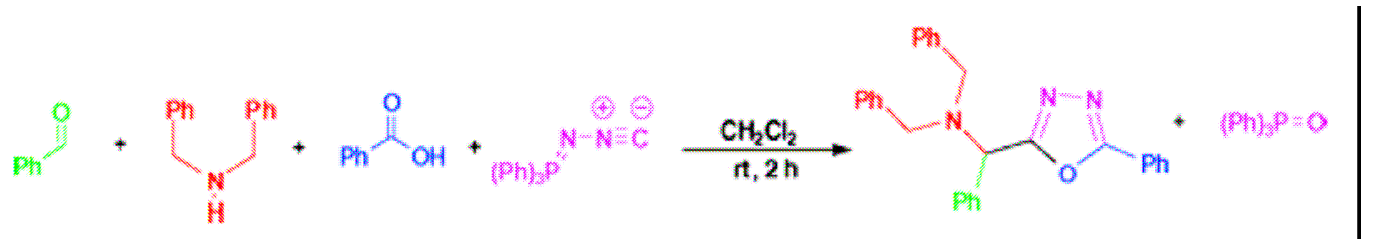

\section{Exercise \#29}

Yoichiro Kuninobu, Takashi Matsuki and Kazuhiko Takai. Rhenium-Catalyzed Synthesis of Indenones by Novel Dehydrative Trimerization of Aryl Aldehydes via C-H Bond Activation. Org. Lett. 2010, 12, 2948-2950.<smiles>[R]c1ccc(C=O)cc1</smiles>

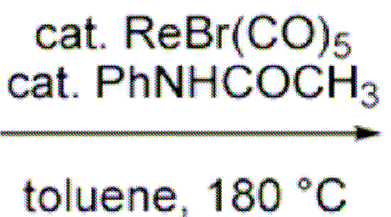<smiles>[R]c1ccc(C2=C(c3ccc([R])cc3)c3cc([R])ccc3C2=O)cc1</smiles>

Exercise \#30

Changkun Li, Hang Zhang, Jiajie Feng, Yan Zhang and Jianbo Wang. Rh(I)-Catalyzed Carbonylative Carbocyclization of Tethered Ene- and Yne-cyclopropenes. Org. Lett. 2010, 12, 3082-3085. 


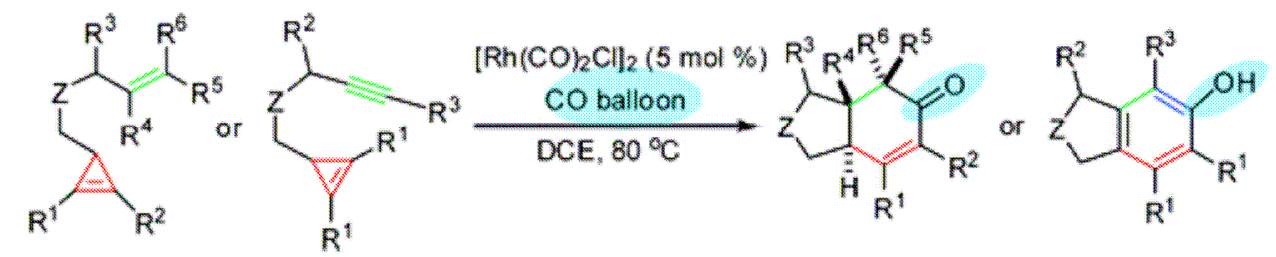

Exercise \#31

Jason P. Burke, Michal Sabat, Diana A. Iovan, William H. Myers and Jason J. Chruma. Exploring the Original Proposed Biosynthesis of (+)-Symbioimine: Remote Exocyclic Stereocontrol in a Type I IMDA Reaction. Org. Lett. 2010, 12, 3192-3195.

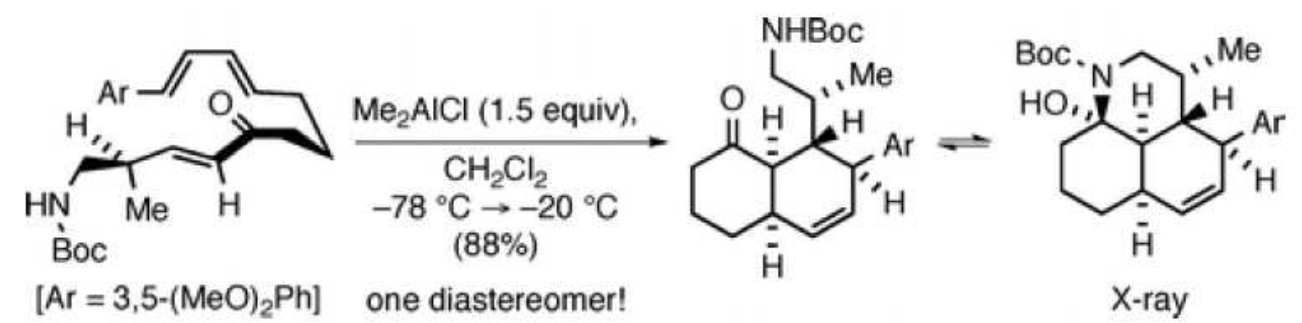

\section{Exercise \#32}

Boris A. Trofimov, Olesya A. Shemyakina, Anastasiya G. Mal’ kina, Igor’ A. Ushakov, Olga N. Kazheva, Grigorii G. Alexandrov and Oleg A. Dyachenko. A Domino Reaction of $\alpha, \beta$-Acetylenic $\gamma$-Hydroxy Nitriles with Arenecarboxylic Acids: An Unexpected Facile Shortcut to 4-Cyano-3(2H)-Furanones. Org. Lett. 2010, 12, 32003203.<smiles>[R]c1ccccc1C(=O)OCC(C)N(C)CC</smiles><smiles>[R][I-]1C=CC=C(C2=C(C#N)C(=O)C([R])([R])O2)C1</smiles>

$$
\mathrm{R}^{1}, \mathrm{R}^{2}=\text { alkyl, cycloalkyl; } \mathrm{R}^{3}=\mathrm{H}, 3-\mathrm{Me}, 4-\mathrm{Me}, 3-\mathrm{F}, 2-\mathrm{Cl}, 3-\mathrm{Cl}, 2-\mathrm{Br}, 3-\mathrm{I}
$$

\section{Exercise \#33}

Sensuke Ogoshi, Akira Nishimura and Masato Ohashi. Nickel-Catalyzed [2+2+2] Cycloaddition of Two Enones and an Alkyne. Org. Lett. 2010, 12, 3450-3452. 
<smiles>CC1C([P+]([O-])(c2ccccc2)c2ccccc2)=C(C(c2ccccc2)c2ccccc2)[C@H](C(=O)c2ccccc2)C(C)C1C(=O)c1ccccc1</smiles>

\section{Exercise \#34}

Curtis A. Seizert, Valentina D. Bumbu and Vladimir B. Birman. A Cope RearrangementBased Route to Hexahydroazulenes. Org. Lett. 2010, 12, 3472-3475.
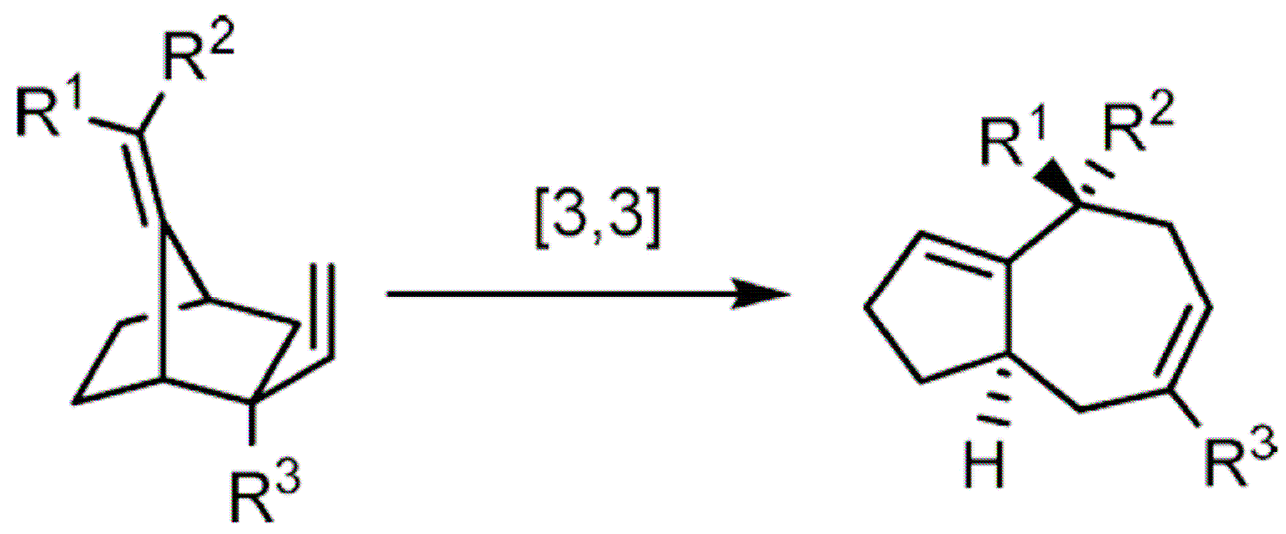

Exercise \#35

Santos Fustero, Silvia Catalan, Maria Sanchez-Rosello, Antonio Simon-Fuentes and Carlos del Pozo. Tandem Asymmetric Michael Reaction-Intramolecular Michael Addition. An Easy Entry to Chiral Fluorinated 1,4-Dihydropyridines. Org. Lett. 2010, 12, 3484-3487.

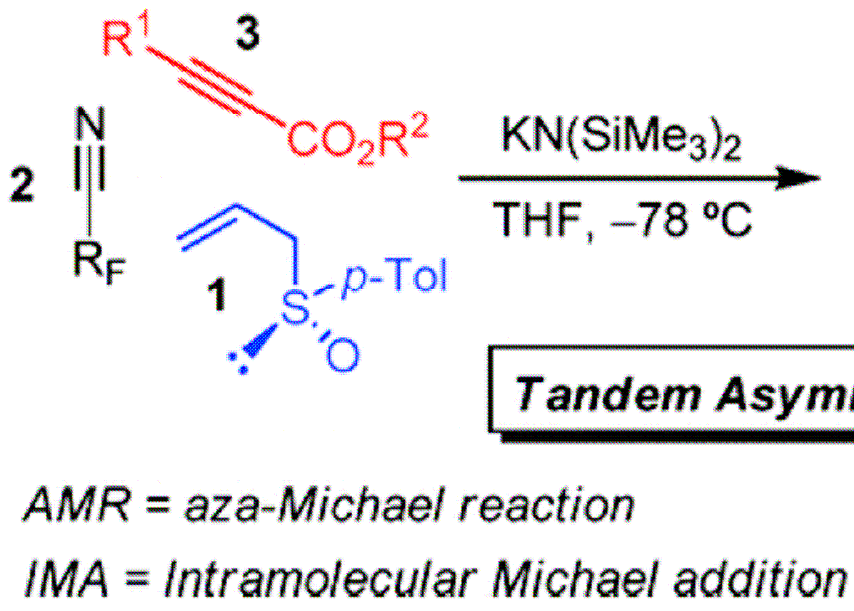

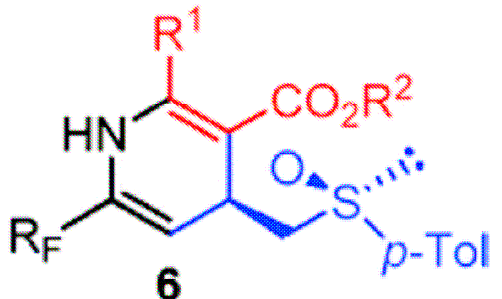

6 


\section{Exercise \#36}

Fanlong Zeng and Howard Alper. One-Step Synthesis of Quinazolino[3,2-

a]quinazolinones via Palladium-Catalyzed Domino Addition/Carboxamidation Reactions. Org. Lett. 2010, 12, 3642-3644.

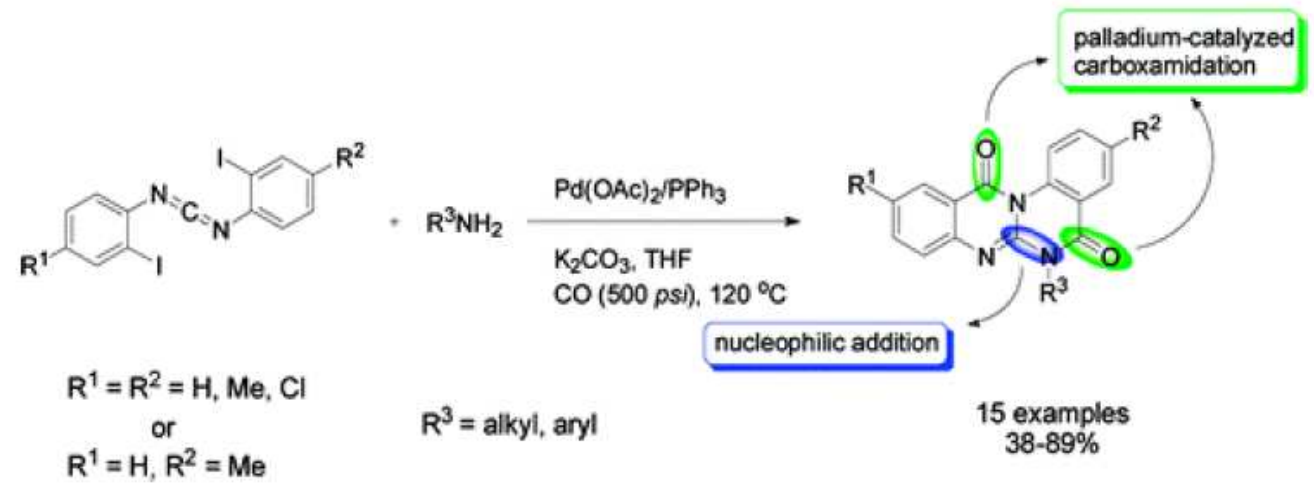

\section{Exercise \#37}

Chun-Ming Yang, Masilamani Jeganmohan, Kanniyappan Parthasarathy and Chien-Hong Cheng. Highly Selective Nickel-Catalyzed Three-Component Coupling of Alkynes with Enones and Alkenyl Boronic Acids: A Novel Route to Substituted 1,3-Dienes. Org. Lett. 2010, 12, 3610-3613.
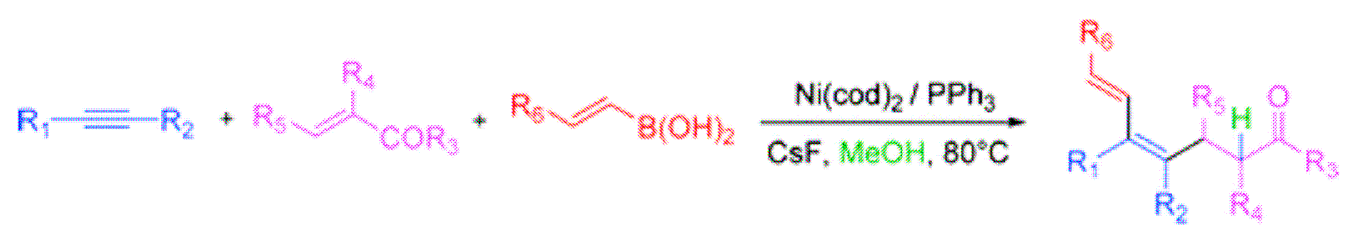

Exercise \#38

Wenteng Chen, Miao Hu, Jianwei Wu, Hongbin Zou and Yongping Yu. Domino Approach for the Synthesis of Pyrrolo[1,2- $\alpha$ ]pyrazine from Vinyl Azides. Org. Lett. 2010, 12, 3863-3865.

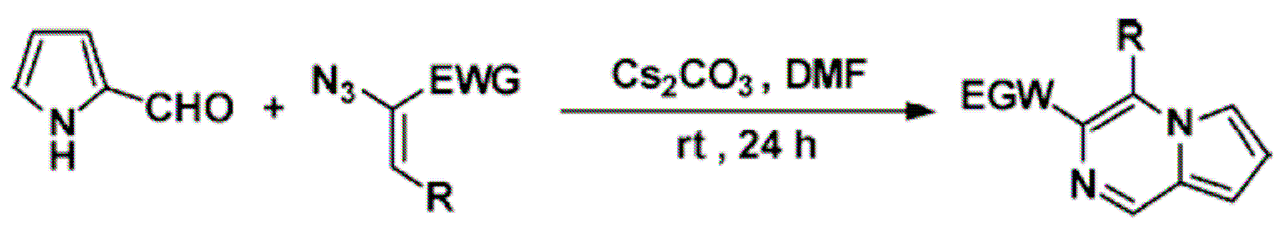

Exercise \#39

Roman S. Borisov, Anatoliy I. Polyakov, Lidia A. Medvedeva, Victor N. Khrustalev, Natalia I. Guranova and Leonid G. Voskressensky. Concise Approach toward Tetrazolo[1,5- $a][1,4]$ benzodiazepines via a Novel Multicomponent Isocyanide-Based Condensation. Org. Lett. 2010, 12, 3894-3897. 


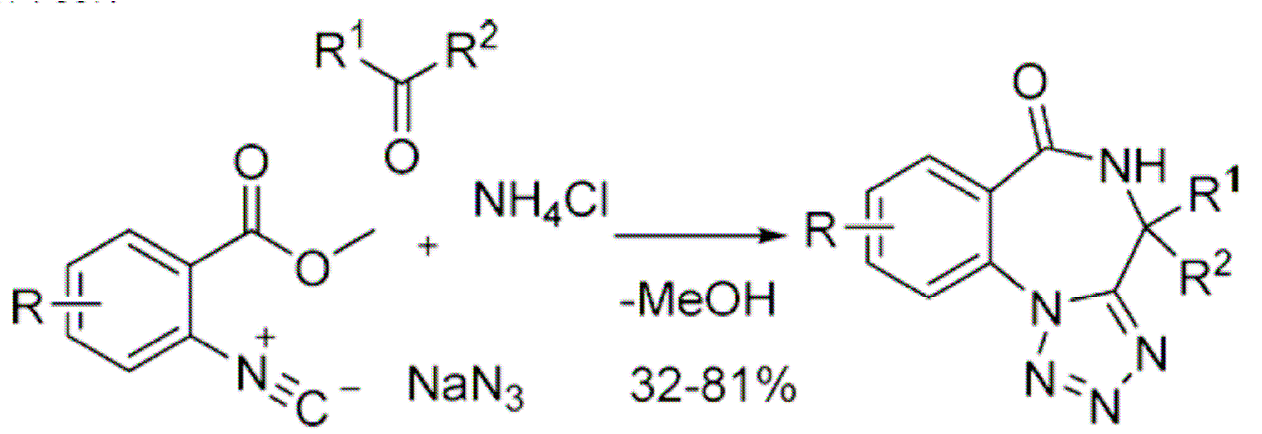

\section{$\mathrm{R}^{1}, \mathrm{R}^{2}=$ Alkyl,cycloalkyl,adamantyl, piperidyl $\mathrm{R}=\mathrm{H}, \mathrm{Br}$, COOMe}

\section{Exercise \#40}

Jun-ichi Matsuo, Mizuki Kawano, Ryosuke Okuno and Hiroyuki Ishibashi. Titanium(IV) Chloride-Mediated Carbon-Carbon Bond-Forming Reaction between 3,3-

Dialkylcyclobutanones and Aldehydes. Org. Lett. 2010, 12, 3960-3962.

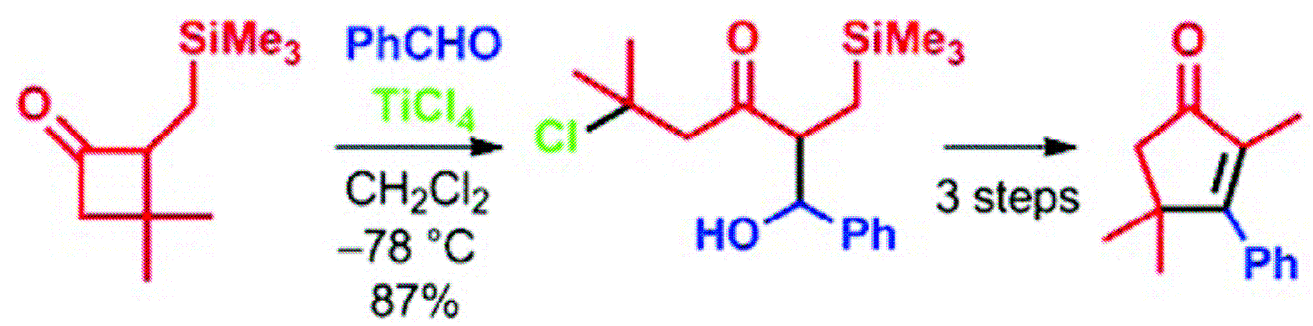

Exercise \#41

Tong Wang and Song Ye. Diastereoselective Synthesis of 6-Trifluoromethyl-5,6dihydropyrans via Phosphine-Catalyzed [4+2] Annulation of $\alpha$-Benzylallenoates with Ketones. Org. Lett. 2010, 12, 4168-4171.

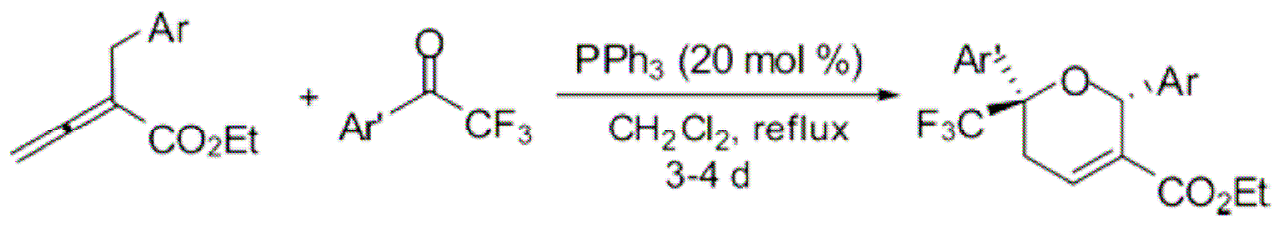

Exercise \#42

Mitsuhiro Yoshimatsu, Hiroyasu Watanabe, and Eri Koketsu. New Cyclization of 4Oxahepta-1,6-diynes Bearing Sulfur and Selenium Functional Groups. Org. Lett. 2010, $12,4192-4194$. 


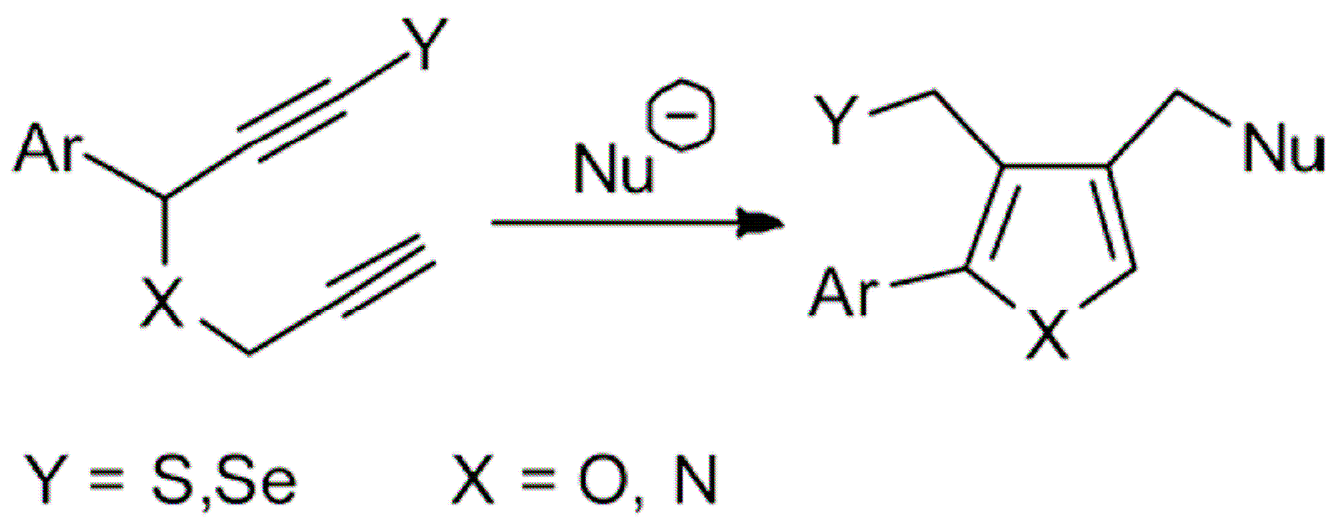

Exercise \#43

Marc Presset, Yoann Coquerel, and Jean Rodriguez. Periselectivity Switch of Acylketenes in Cycloadditions with 1-Azadienes: Microwave-Assisted Diastereoselective Domino Three-Component Synthesis of $\alpha$-Spiro- $\delta$-lactams. Org. Lett. 2010, 12, 42124215.

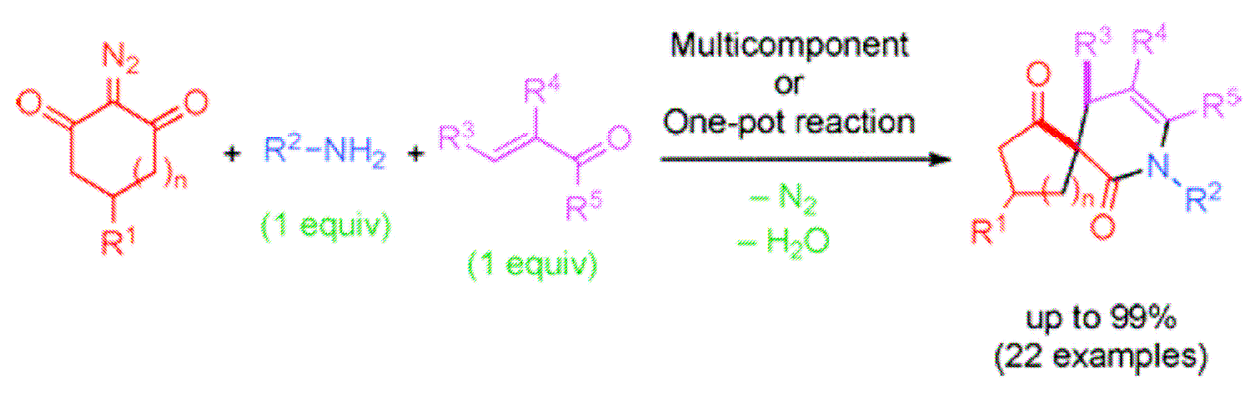

\section{Exercise \#44}

Erica Benedetti, Gilles Lemiere, Laure-Lise Chapellet, Andrea Penoni, Giovanni Palmisano, Max Malacria, Jean-Philippe Goddard, and Louis Fensterbank. Gold(I)Catalyzed Cyclization of $\beta$-Allenylhydrazones: An Efficient Synthesis of Multisubstituted N-Aminopyrroles. Org. Lett. 2010, 12, 4396-4399.
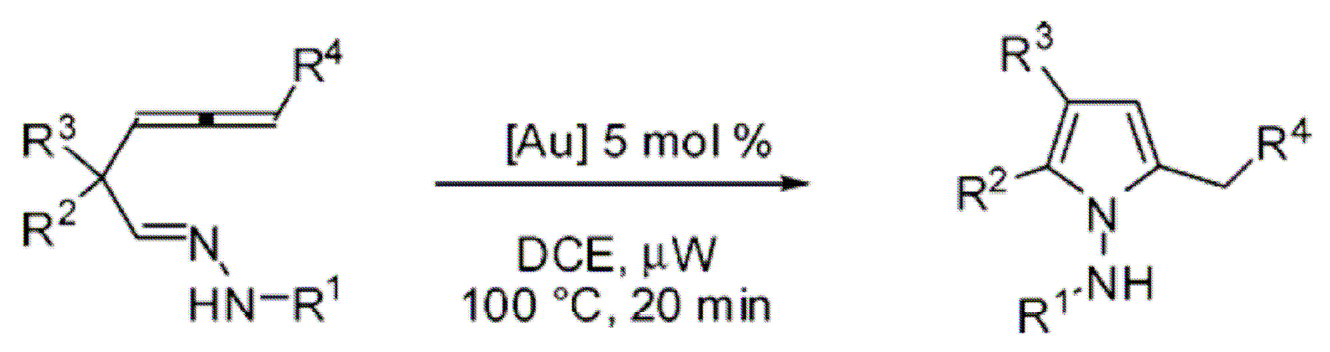

\section{Exercise \#45}

Kenichi Ogata, Yuka Atsuumi, and Shin-ichi Fukuzawa. Nickel-Catalyzed RingOpening Three-Component Coupling of Methylenecyclopropane with Aldehydes and Silanes. Org. Lett. 2010, 12, 4536-4539. 


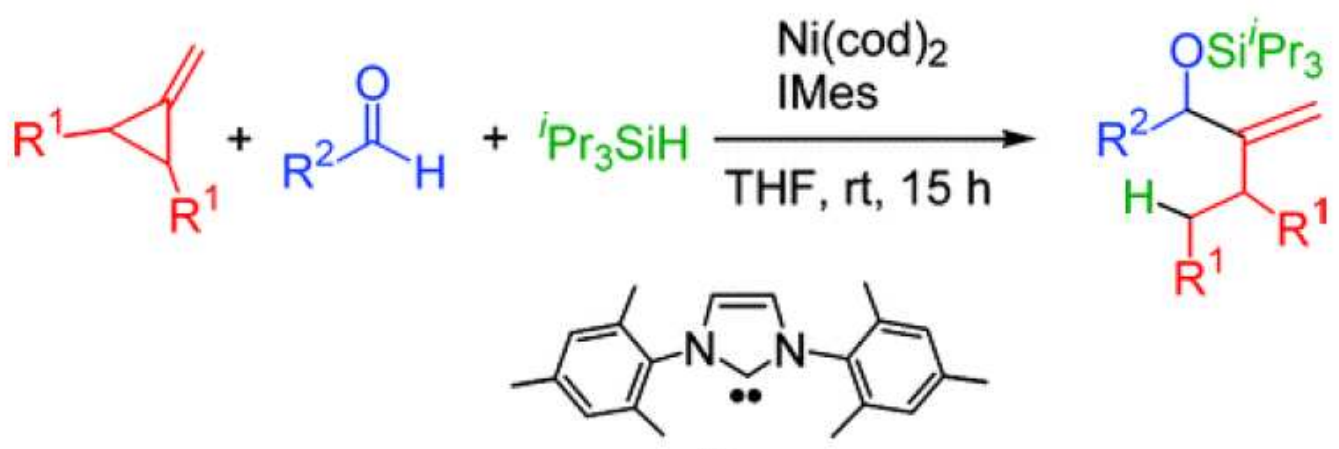

IMes

Exercise \#46

Lei Miao, Imranul Haque, Maria R. Manzoni, Weng Siong Tham, and Sherry R.

Chemler. Diastereo- and Enantioselective Copper-Catalyzed Intramolecular

Carboamination of Alkenes for the Synthesis of Hexahydro-1Hbenz[f]indoles. Org. Lett.

2010, 12, 4739-4741.

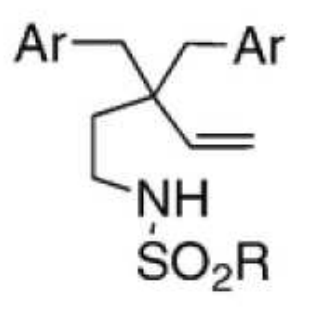

$\mathrm{Cu}(\mathrm{OTf})_{2}(20 \mathrm{~mol} \%)$ $(R, R)-\mathrm{Ph}-$ box $(25 \mathrm{~mol} \%)$

$\mathrm{K}_{2} \mathrm{CO}_{3}, \mathrm{PhCF}_{3}$

$120^{\circ} \mathrm{C}, 24 \mathrm{~h}$

$89-99 \%$

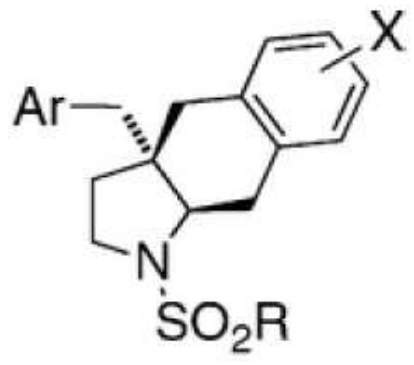

$82-97 \%$ ee

\section{Exercise \#47}

Sabrina Peixoto, Tuan Minh Nguyen, David Crich, Bernard Delpech, and Christian Marazano. One-Pot Formation of Piperidine- and Pyrrolidine-Substituted Pyridinium Salts via Addition of 5-Alkylaminopenta-2,4-dienals to $\mathrm{N}$-Acyliminium Ions: Application to the Synthesis of ( \pm )-Nicotine and Analogs. Org. Lett. 2010, 12, 4760-4763.

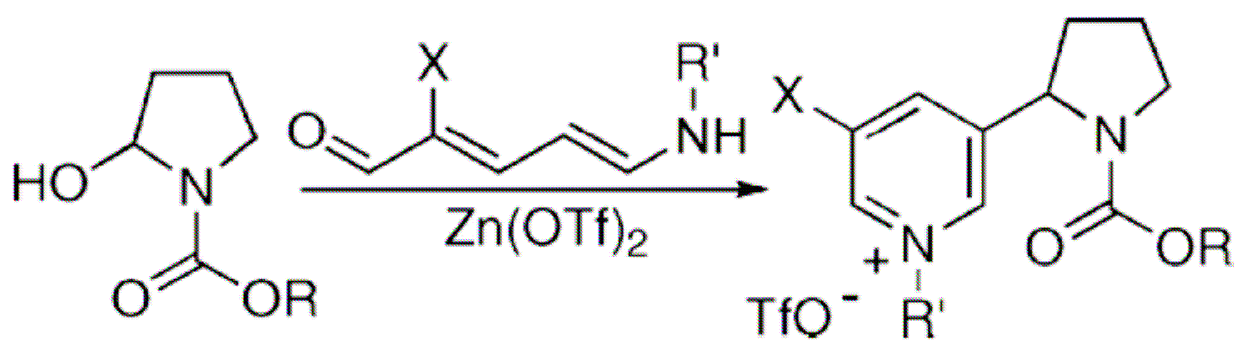




\section{Exercise \#48}

Ryosuke Saijo, Yuri Hagimoto, and Masami Kawase. New Synthesis of 3-

Trifluoromethylpyrroles by Condensation of Mesoionic 4-Trifluoroacetyl-1,3-oxazolium5-olates with Phosphorus Ylides. Org. Lett. 2010, 12, 4776-4779.
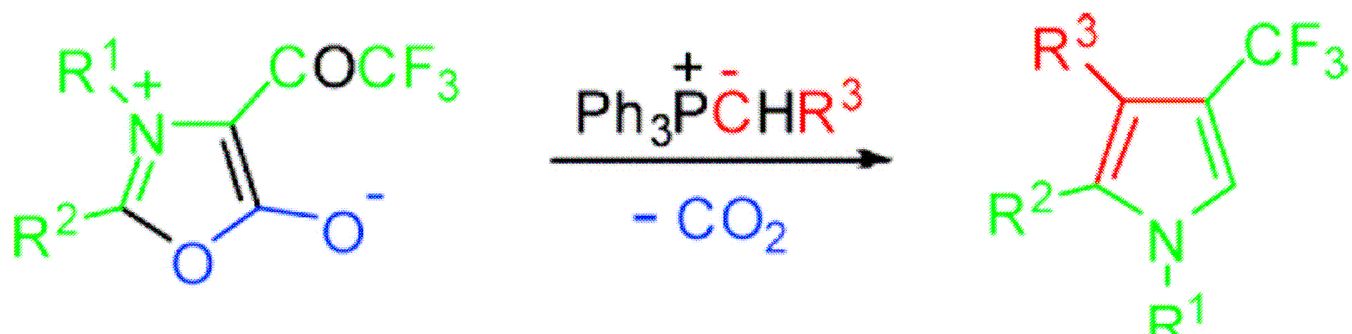

\section{Exercise \#49}

Zhiyuan Chen and Jie Wu. Efficient Generation of Biologically Active $H$-Pyrazolo[5,1a]isoquinolines via Multicomponent Reaction. Org. Lett. 2010, 12, 4856-4859.

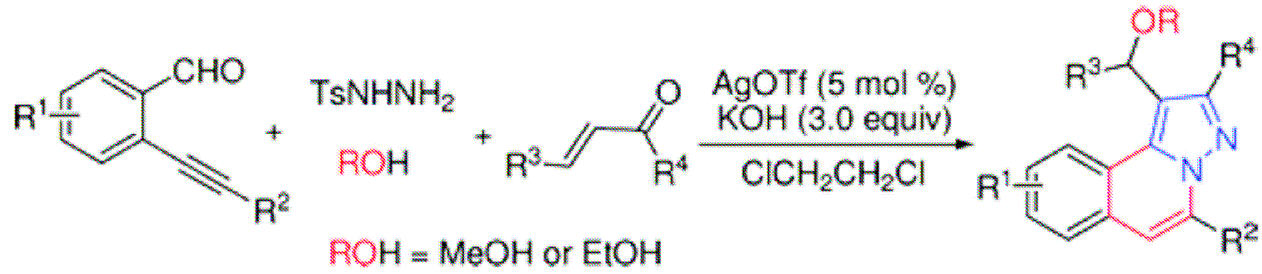

Exercise \#50

Chao Feng, Yong Liu, Shengming Peng, Qi Shuai, Guojun Deng, and Chao-Jun Li.

Ruthenium-Catalyzed Tertiary Amine Formation from Nitroarenes and Alcohols. Org.

Lett. 2010, 12, 4888-4891.

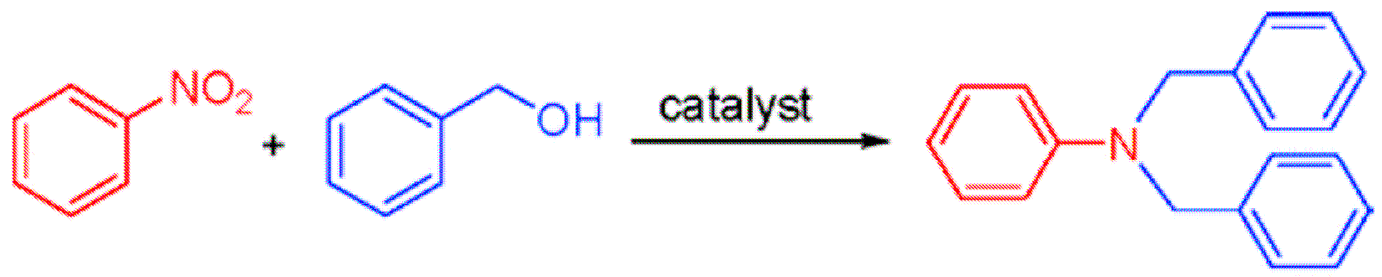

\section{borrowing hydrogen strategy}

\section{Exercise \#51}

Marie S. T. Morin, Daniel J. St-Cyr, and Bruce A. Arndtsen.

Horner-Wadsworth-Emmons Reagents as Azomethine Ylide Analogues: Pyrrole

Synthesis via (3 + 2) Cycloaddition. Org. Lett. 2010, 12, 4916-4919. 


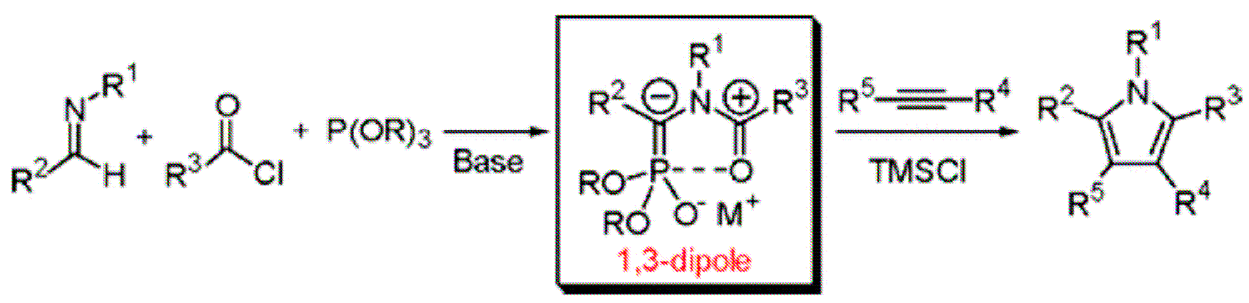

\section{Exercise \#52}

Xiao-Yang Guan, Yin Wei, and Min Shi. Phosphine-Catalyzed Tandem Reaction of Allenoates with Nitroalkenes. Org. Lett. 2010, 12, 5024-5027.

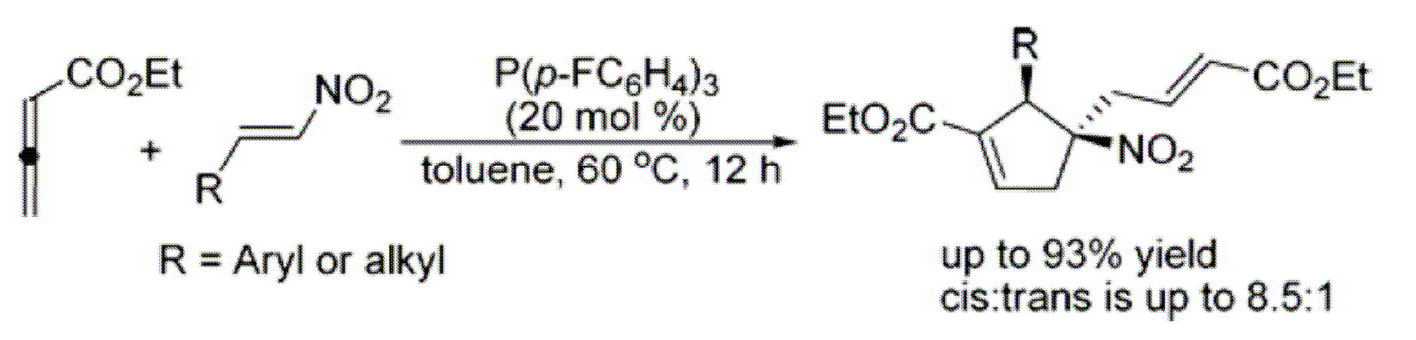

Exercise \#53

Shuichi Suzuki, Kozo Fukui, Akira Fuyuhiro, Kazunobu Sato, Takeji Takui, Kazuhiro Nakasuji, and Yasushi Morita. Hexaazaphenalene Derivatives: One-Pot Synthesis, Hydrogen-Bonded Chiral Helix, and Fluorescence Properties. Org. Lett. 2010, 12, 50365039.

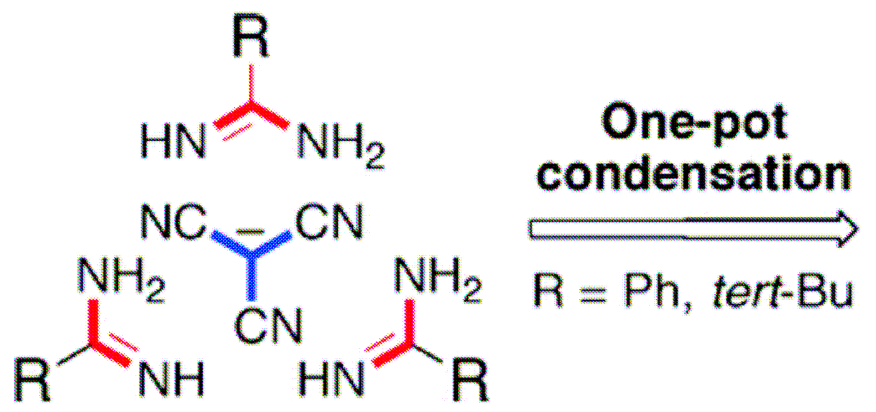<smiles>[R]C1=Nc2nc([R])nc3nc([R])nc(c23)N1</smiles>

Exercise \#54

Jian Cao and Xian Huang. Facile Synthesis of Tetrahydro- $1 H$-isoindolones via a Sequential Three-Component Copper-Catalyzed Coupling/Propargyl-Allenyl Isomerization/[4 + 2] Cyclization Reaction. Org. Lett. 2010, 12, 5048-5051.

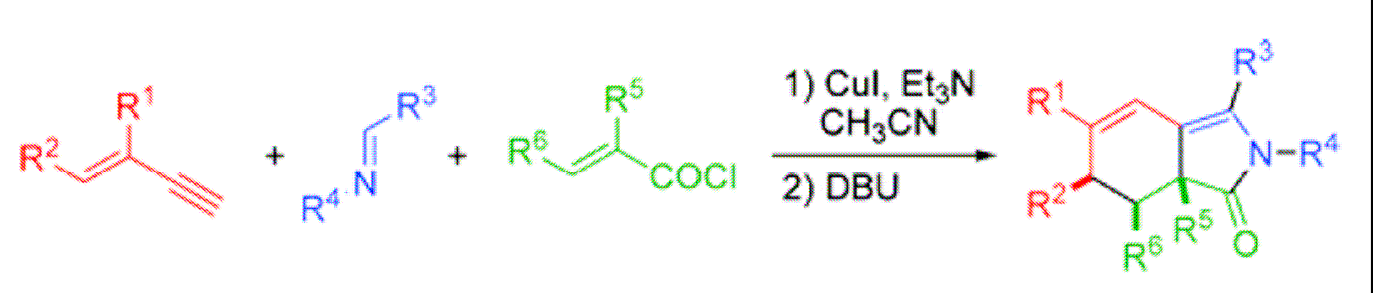




\section{Exercise \#55}

Yoshiaki Takada, Kenichi Nomura, and Seijiro Matsubara. Preparation of a Cycloheptane Ring from a 1,2-Diketone with High Stereoselectivity.

Org. Lett. 2010, 12, 5204-5205.<smiles>[R7]C=CC(=O)C(=O)/C=C/[R]</smiles><smiles>[R7]C1=CC([R7])C([R7])C=C([O])C1</smiles>

Exercise \#56

Huihui Fang, Xiaoyu Wu, Linlin Nie, Xiaoyang Dai, Jie Chen, Weiguo Cao, and Gang Zhao. Diastereoselective Syntheses of Indoloquinolizidines by a

Pictet-Spengler/Lactamization Cascade. Org. Lett. 2010, 12, 5366-5369.

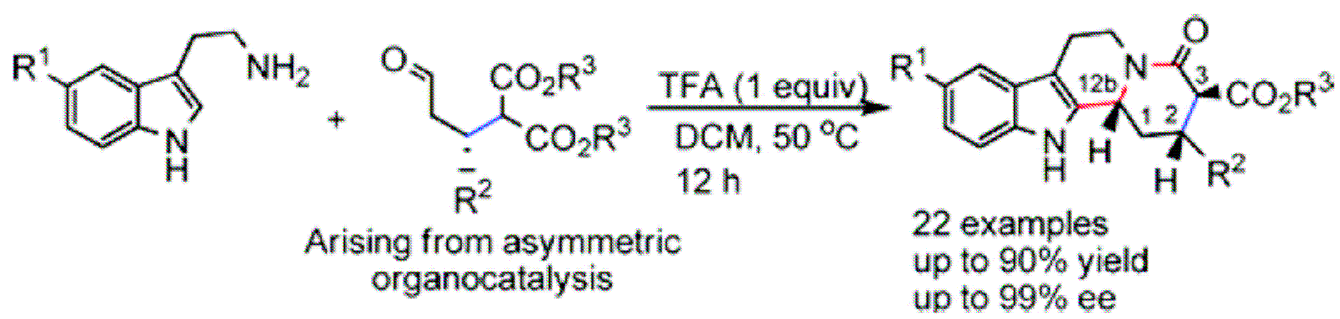

\section{Exercise \#57}

Huanfeng Jiang, Huawen Huang, Hua Cao, and Chaorong Qi. TBHP/I2-Mediated Domino Oxidative Cyclization for One-Pot Synthesis of Polysubstituted Oxazoles. Org. Lett. 2010, 12, 5561-5563.

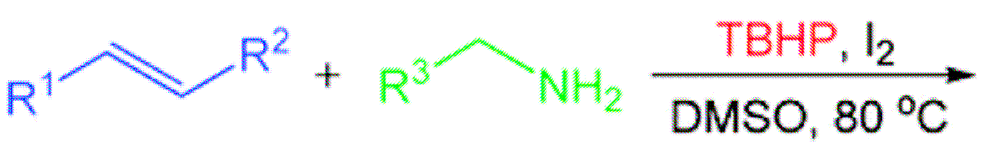

$$
\mathrm{R}^{1}, \mathrm{R}^{3}=\text { aryl } \mathrm{R}^{2}=\mathrm{H} \text { or aryl }
$$<smiles>[R]c1nc([R])c([R])o1</smiles> 
Exercise \#58

Fanlong Zeng and Howard Alper. Palladium-Catalyzed Domino Ring-

Opening/Carboxamidation Reactions of $N$-Tosyl Aziridines and 2-Iodothiophenols: A

Facile and Efficient Approach to 1,4-Benzothiazepin-5-ones. Org. Lett. 2010, 12, 55675569.

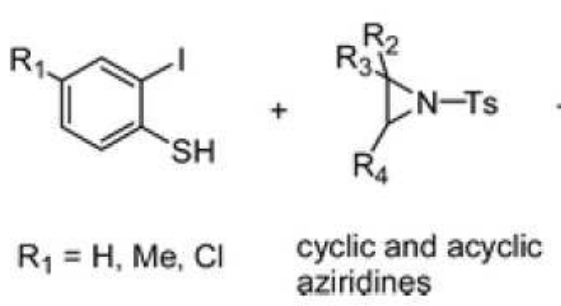

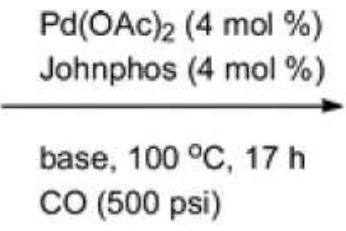

CO (500 psi)

\section{Exercise \#59}

Yu Shibata, Keiichi Noguchi, and Ken Tanaka. Cationic Rhodium(I) Complex-

Catalyzed Cotrimerization of Propargyl Esters and Arylacetylenes Leading to Substituted Dihydropentalenes. Org. Lett. 2010, 12, 5596-5599.

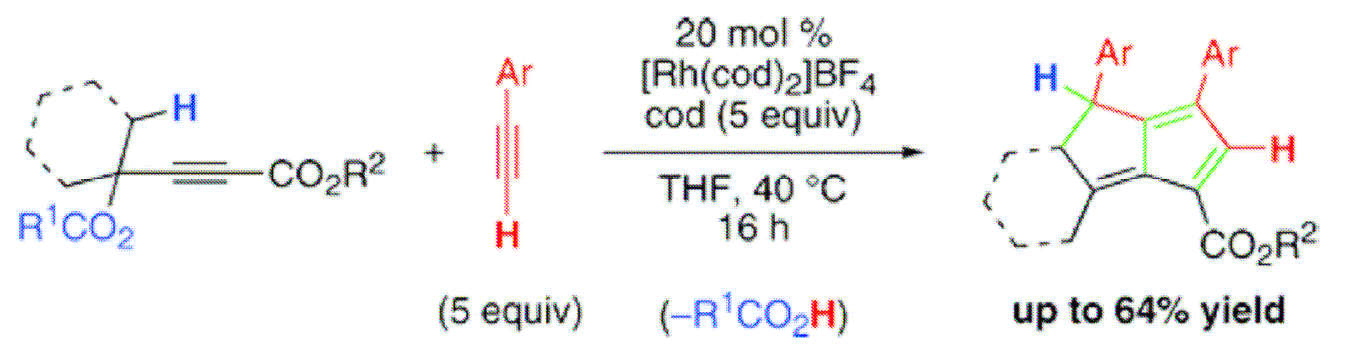

\section{Exercise \#60}

Chin-Chau Chen, Lin-Yu Chin, Shyh-Chyun Yang, and Ming-Jung Wu. Synthetic Development and Mechanistic Study on Pd(II)-Catalyzed Cyclization of Enediynes to Benzo[a]carbazoles. Org. Lett. 2010, 12, 5652-5655.<smiles>[R3]C#Cc1ccccc1C#Cc1cc([R2])cc([R7])c1N(C)C</smiles>

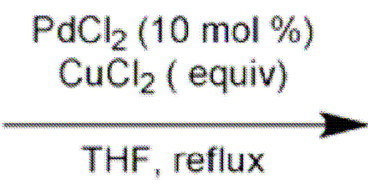

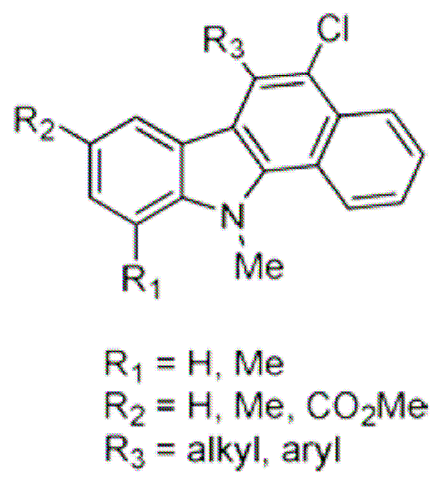

Exercise \#61 
Zhengxin Cai and Michael Harmata. Studies Directed toward the Synthesis of Hamigeran B: A Catalytic Oxidative Cyclization. Org. Lett. 2010, 12, 5668-5670.<smiles>C=Cc1c(C=CC(C)C(C)C)cc(C)cc1C=C(C)C(=O)C(=O)CC(C)C</smiles>

\section{Exercise \#62}

Eduardo Sanchez-Larios, Janice M. Holmes, Crystal L. Daschner, and Michel Gravel. NHC-Catalyzed Spiro Bis-Indane Formation via Domino Stetter-Aldol-Michael and Stetter-Aldol-Aldol Reactions. Org. Lett. 2010, 12, 5772-5775.

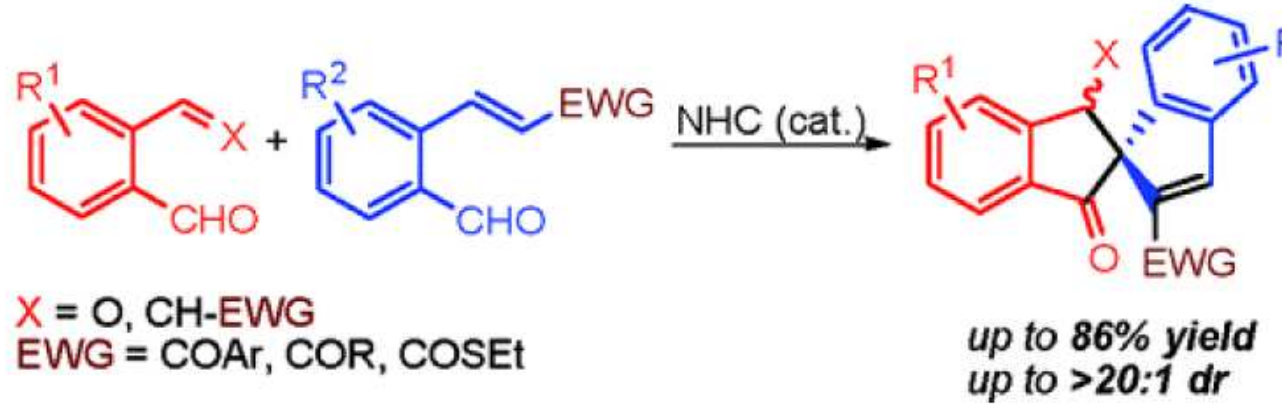

\section{Exercise \#63}

Zhiyuan Chen, Danqing Zheng, and Jie Wu. A Facile Route to Polysubstituted Indoles via Three-Component Reaction of 2-Ethynylaniline, Sulfonyl Azide, and Nitroolefin.

Org. Lett. 2011, 13, 848-851.

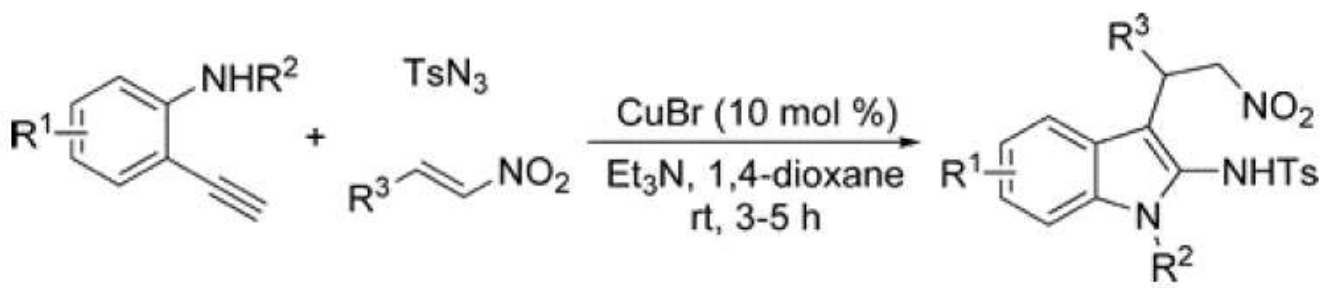

\section{Exercise \#64}

Guangwei Yin, Yuanxun Zhu, Li Zhang, Ping Lu, and Yanguang Wang. Preparation of Allenephosphoramide and Its Utility in the Preparation of 4,9-Dihydro- $2 \mathrm{H}$ benzo[f]isoindoles. Org. Lett. 2011, 13, 940-943. 


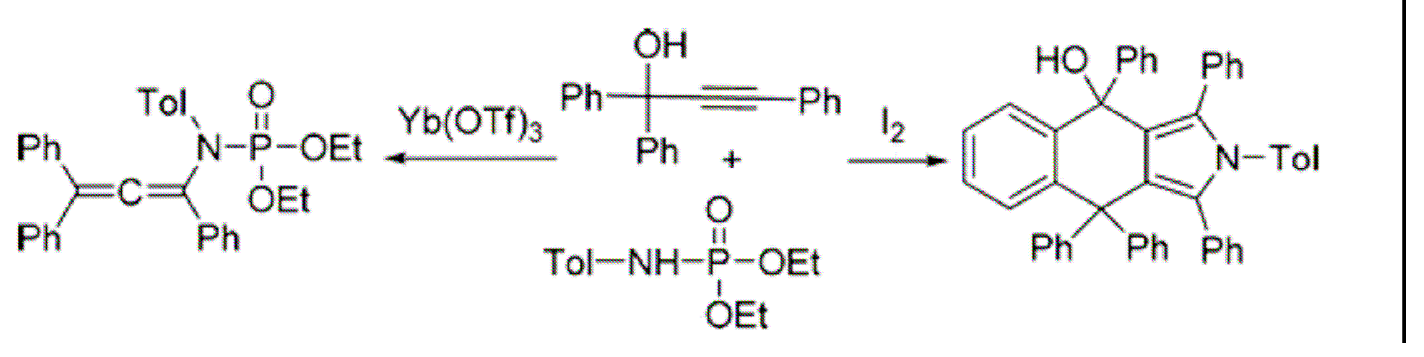

Exercise \#65

Andrew C. Stevens, Cory Palmer, and Brian L. Pagenkopf. The Formal [4+3]

Cycloaddition between Donor-Acceptor Cyclobutanes and Nitrones.

Org. Lett. 2011, 13, 1528-1531.

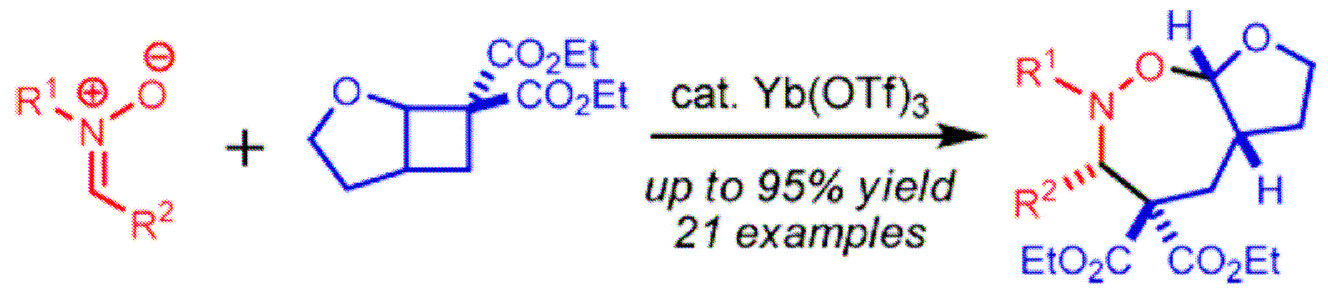

Exercise \#66

Leah Cleary, Hoseong Yoo, and Kenneth J. Shea. Microwave Assisted Synthesis of Bridgehead Alkenes. Org. Lett. 2011, 13, 1781-1783.
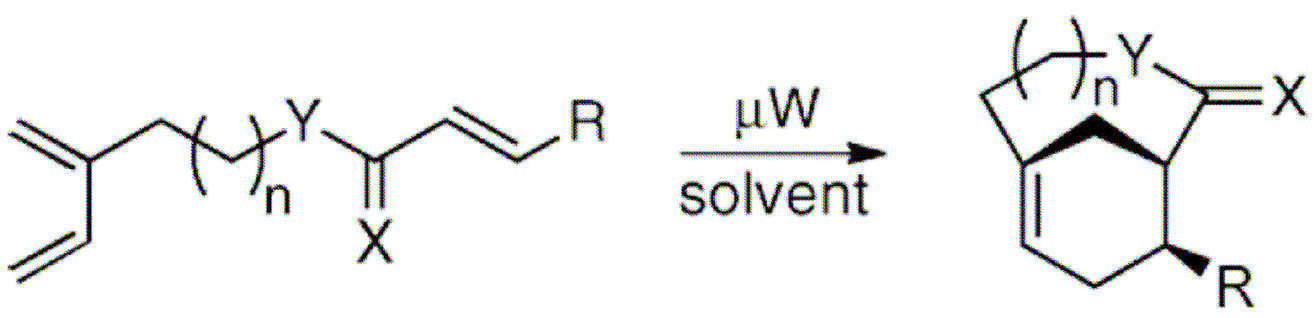

Exercise \#67

Svitlana Kulyk, William G. Dougherty, Jr., W. Scott Kassel, Michael J. Zdilla, and Scott McN. Sieburth. Intramolecular Pyridone/Enyne Photocycloaddition: Partitioning of the [4+4] and [2+2] Pathways. Org. Lett. 2011, 13, 2180-2183. 


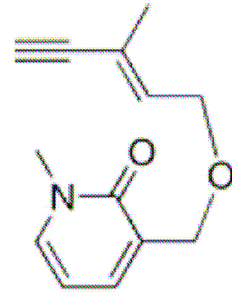

head-to-tail

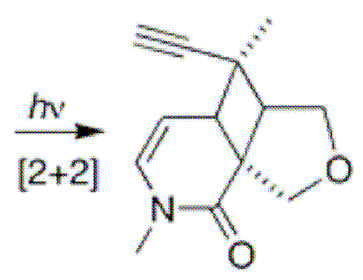

head-to-head

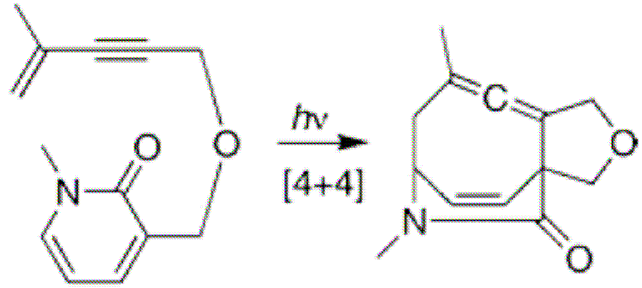

Exercise \#68

Ming Yao and Chong-Dao Lu. Three-Component Reactions of Sulfonylimidates, Silyl Glyoxylates and $N$-tert-Butanesulfinyl Aldimines: An Efficient, Diastereoselective, and Enantioselective Synthesis of Cyclic N-Sulfonylamidines. Org. Lett. 2011, 13, 27822785 .

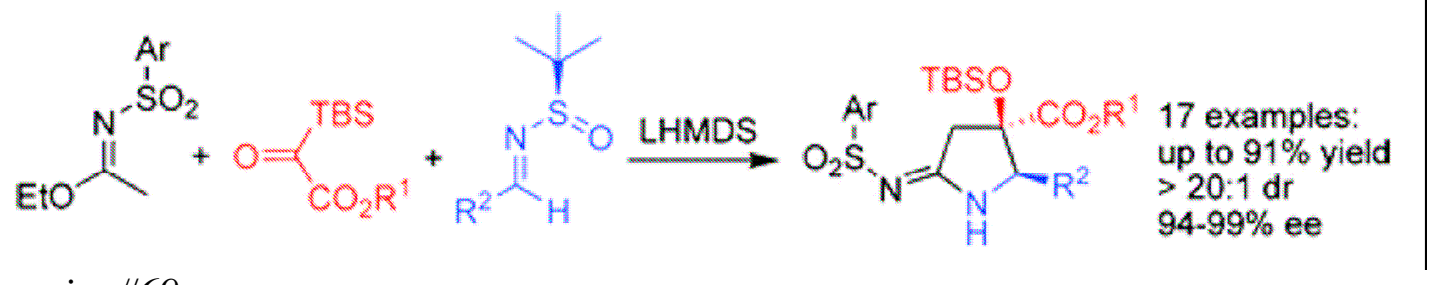

Exercise \#69

Marta L. Lage, Israel Fernandez, Miguel A. Sierra, and M. Rosario Torres. Trapping Intermediates in an [8+2] Cycloaddition Reaction with the Help of DFT Calculations. Org. Lett. 2011, 13, 2892-2895.<smiles></smiles><smiles>CC(C=O)c1ccccc1</smiles><smiles>[R]/C(=C\c1ccccc1)N([Z18])c1ccccc1</smiles><smiles>CCN1C(=O)[C@H](c2ccccc2)[C@H]2C=CC=CC21</smiles>

trapped zwitterionic intermediate

Exercise \#70

Masahiro Yoshida, Chiyuki Sugimura, and Kozo Shishido. Diastereoselective Construction of 7-Methylenebicyclo[3.2.1]oct-3-en-2-one Derivatives by PalladiumCatalyzed Cyclization of Propargylic Acetates with 2-Oxocyclohex-3-enecarboxylates. Org. Lett. 2011, 13, 3482-3485. 


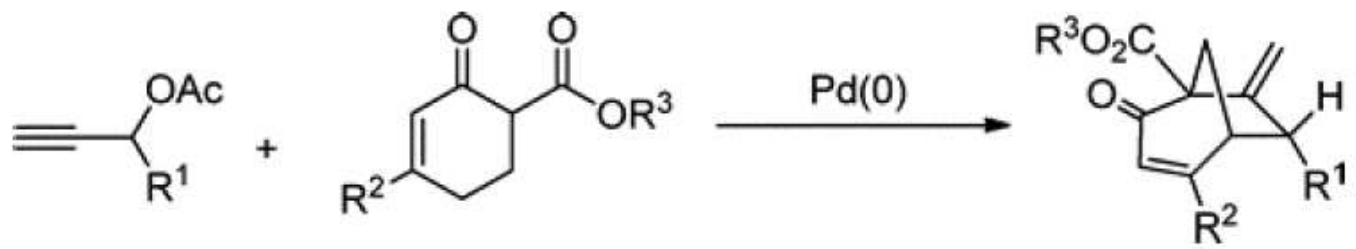

Exercise \#71

Tomoya Miura, Yusuke Mikano, and Masahiro Murakami. Nickel-Catalyzed Synthesis of 1,3,5-Trisubstituted Hydantoins from Acrylates and Isocyanates. Org. Lett. 2011, 13, $3560-3563$.

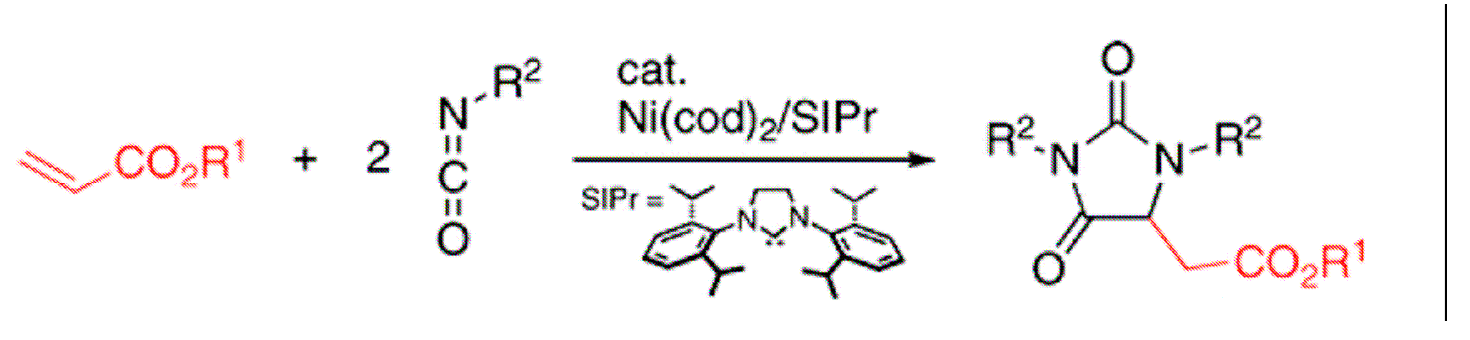

Exercise \#72

Francisco J. Fananas, Tamara Arto, Abraham Mendoza, and Felix Rodriguez. Synthesis of 2,5-Dihydropyridine Derivatives by Gold-Catalyzed Reactions of $\beta$-Ketoesters and Propargylamines. Org. Lett. 2011, 13, 4184-4187.

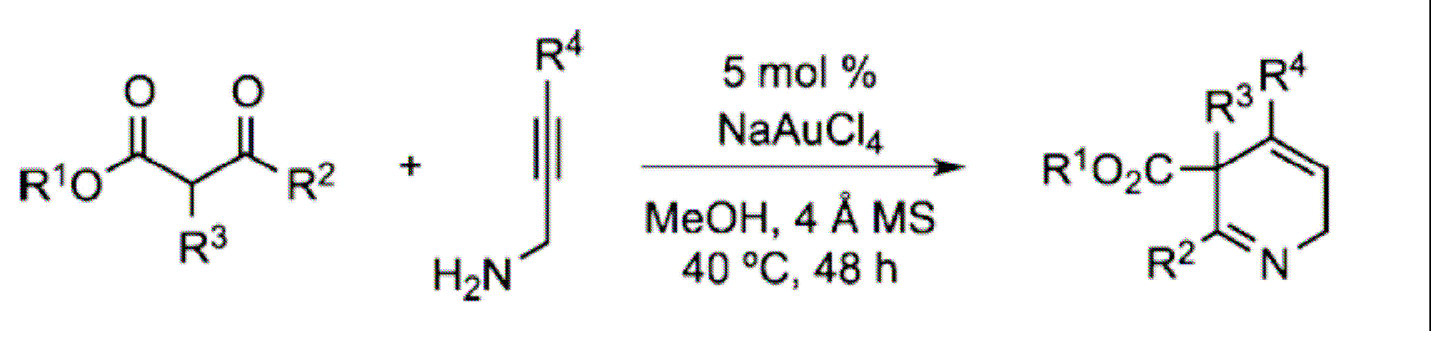

Exercise \#73

Amanda L. Gibeau and John K. Snyder. Indium(III)-Catalyzed Hydrative Cyclization of 1,7-Diynyl Ethers. Org. Lett. 2011, 13, 4280-4283.

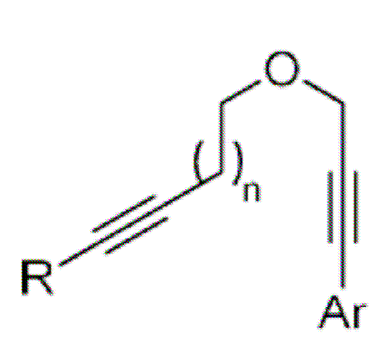

Exercise \#74
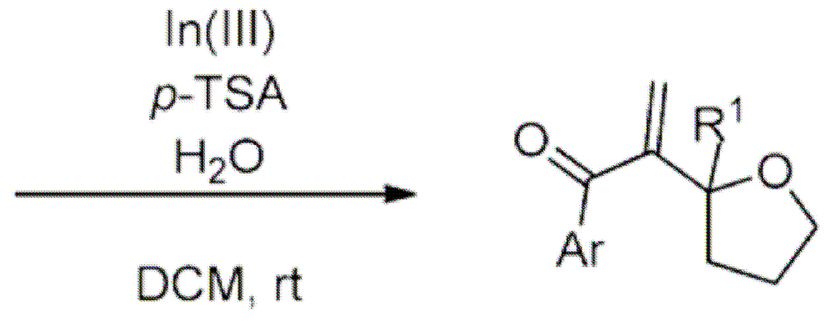

10 examples

up to $66 \%$ yield 
Wen Wang, Yongwen Shen, Xu Meng, Mingming Zhao, Yongxin Chen, and Baohua Chen. Copper-Catalyzed Synthesis of Quinoxalines with $o$-Phenylenediamine and Terminal Alkyne in the Presence of Bases. Org. Lett. 2011, 13, 4514-4517.<smiles>Nc1ccccc1-c1ccccc1</smiles>

Exercise \#75

Chhanda Mukhopadhyay, Paramita Das, and Ray J. Butcher. An Expeditious and Efficient Synthesis of Highly Functionalized [1,6]-Naphthyridines under Catalyst-Free Conditions in Aqueous Medium. Org. Lett. 2011, 13, 4664-4667.<smiles>[R]C(C)=O</smiles><smiles>C1CCNCC1</smiles>
1
2

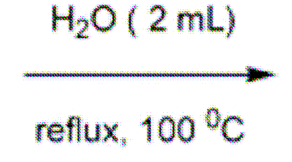
reflux, $100^{\circ} \mathrm{C}$

3<smiles>[R]C1=CC([R])(C)Nc2c(C#N)c(N3CCCCCCC3)nc(N)c21</smiles>

4

Exercise \#76

Pierre Fourgeaud, Bénédicte Daydé, Jean-Noël Volle, Jean-Pierre Vors, Arie Van der Lee, Jean-Luc Pirat, and David Virieux. Highly Convergent Synthesis of Chiral Bicyclophosphinates by Domino Hydrophosphinylation/Michael/Michael Reaction. Org. Lett. 2011, 13, 5076-5079.

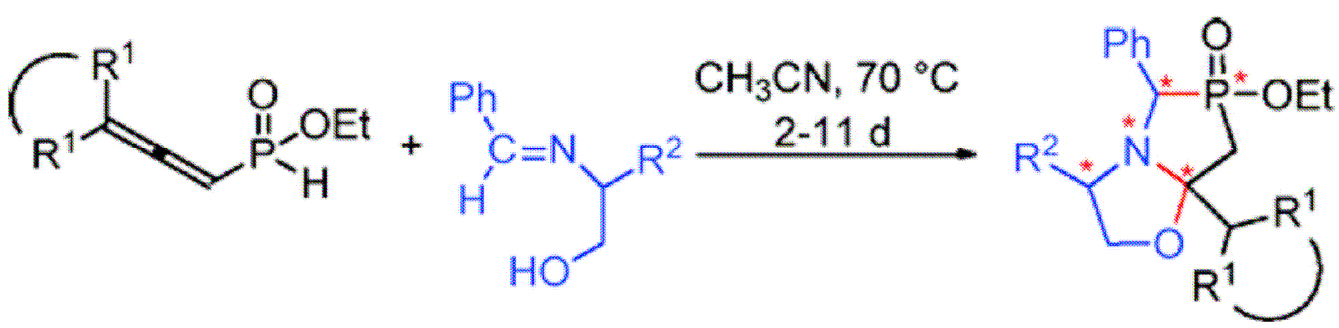

Exercise \#77

Hui Lv, Junming Mo, Xinqiang Fang, and Yonggui Robin Chi. Formal Diels-Alder Reactions of Chalcones and Formylcyclopropanes Catalyzed by Chiral N-Heterocyclic Carbenes. Org. Lett. 2011, 13, 5366-5369. 


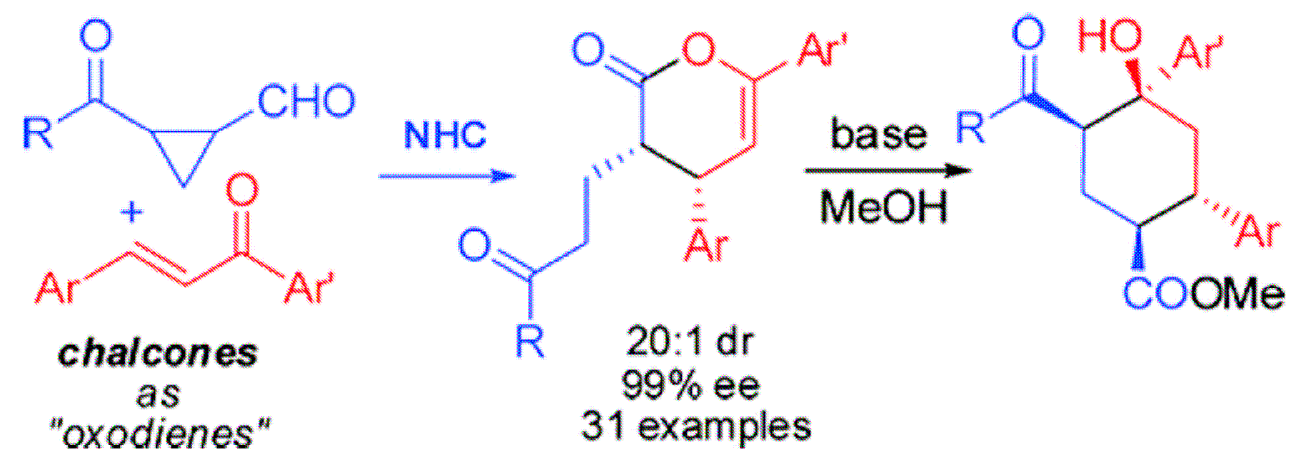

Exercise \#78

Deborah A. Smithen, T. Stanley Cameron, and Alison Thompson. One-Pot Synthesis of Asymmetric Annulated Bis(pyrrole)s. Org. Lett. 2011, 13, 5846-5849.<smiles>[R]c1c[nH]c(C)c1[R]</smiles>

$\mathrm{R}^{1}, \mathrm{R}^{2}=\mathrm{Alkyl}$ $\mathbf{R}^{3}=$ Alkyl or Aryl

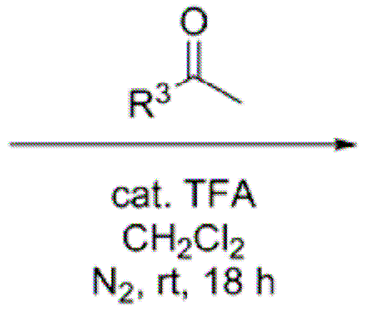

$48-91 \%$<smiles>[R]c1c(C)[nH]c(C2([R])CC([R])(C)c3c([R])c([R])c(C)n32)c1[R]</smiles>

16 examples

\section{Exercise \#79}

Yong Luo and Jie Wu. A New Insight into Palladium-Catalyzed Reaction of 2Alkynylphenol with Carbon Monoxide. Org. Lett. 2011, 13, 5858-5861.

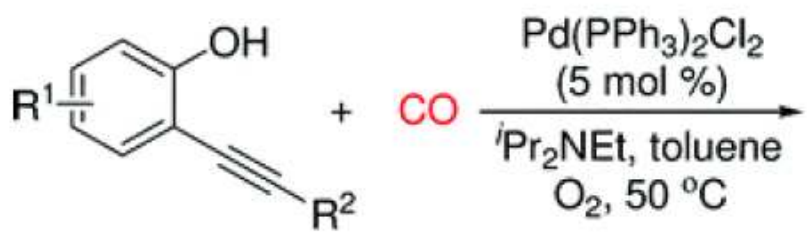

\section{Exercise \#80}

Necdet Coşkun, Jingxiang Ma, Saeed Azimi, Christian Gärtner, and Ihsan Erden. 1,2Dihydropentalenes from Fulvenes by $[6+2]$ Cycloadditions with 1Isopropenylpyrrolidine. Org. Lett., 2011, 13 (22), pp 5952-5955. 


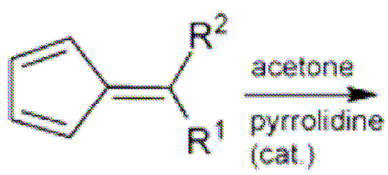<smiles>[R]C([R2])c1ccccc1</smiles><smiles>[R]C1([R])CC(C)=C2C=CC=C21</smiles>

Exercise \#81

Srimanta Guin, Tuhin Ghosh, Saroj Kumar Rout, Arghya Banerjee, and Bhisma K. Patel. $\mathrm{Cu}$ (II) Catalyzed Imine C-H Functionalization Leading to Synthesis of 2,5-Substituted 1,3,4-Oxadiazoles. Org. Lett. 2011, 13, 5976-5979.<smiles>[R][R]1ccc(C(=O)N/N=C/c2ccc([R])cc2)cc1</smiles>

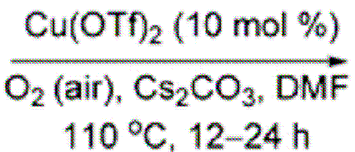<smiles>[R][R]1ccc(-c2nnc(-c3ccc([R])cc3)o2)cc1</smiles>

Exercise \#82

Gopal Sathishkannan and Kannupal Srinivasan. Highly Diastereoselective Synthesis of 1-Pyrrolines via SnCl4-Promoted [3 + 2] Cycloaddition between Activated DonorAcceptor Cyclopropanes and Nitriles. Org. Lett. 2011, 13, 6002-6005.<smiles>CCOC(=O)C1(C(=O)Br)[C@@H](Br)[C@@H]1Br</smiles><smiles>[R]C#[N+][R6]</smiles><smiles>[R]C1=N[C@@H]([Al])[C@@H](C(=O)[Te])[C@]1(C)C(=O)OCC</smiles>

$48-90 \%$

\section{Exercise \#83}

Francois Grillet, Chaofeng Huang, and Kay M. Brummond. An Allenic Pauson-Khand Approach to 6,12-Guaianolides. Org. Lett. 2011, 13, 6304-6307.
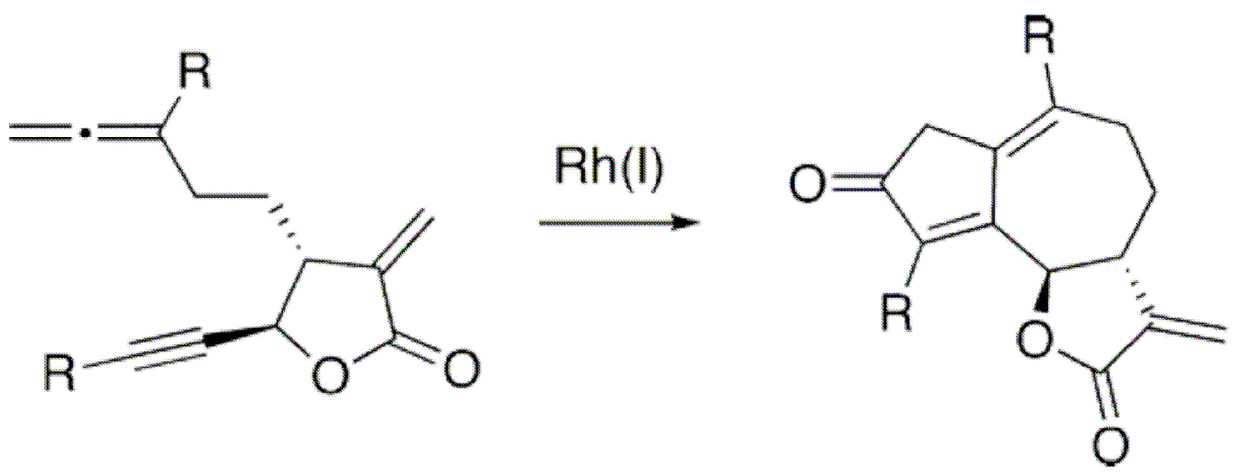

Exercise \#84

Bao Hu, Zhao Wang, Ning Ai, Jie Zheng, Xing-Hai Liu, Shang Shan, and Zhongwen 
Wang. Catalyst-Free Preparation of 1,2,4,5-Tetrasubstituted Imidazoles from a Novel Unexpected Domino Reaction of 2-Azido Acrylates and Nitrones. Org. Lett. 2011, 13, $6362-6365$.<smiles>[R]C=C([R])N</smiles><smiles>[R]c1nc([R])n([R])c1[R]</smiles>

\section{6 examples in $21-98 \%$}

Exercise \#85

Sophie M.-C. Pelletier, Peter C. Ray, and Darren J. Dixon. Diastereoselective Synthesis of 1,3,5-Trisubstituted 4-Nitropyrrolidin-2-ones via a Nitro-Mannich/Lactamization Cascade. Org. Lett. 2011, 13, 6406-6409.<smiles>COC(=O)C(C[N+](=O)[O-])C(C)C</smiles>

$\mathbf{R}$<smiles>[R]C=CC#CN([As])C[R]</smiles>

Exercise \#86

Jian Cao, Yongping Xu, Yulong Kong, Yuming Cui, Ziqiang Hu, Guanhai Wang, Yuan Deng, and Guoqiao Lai. Synthesis of $\delta$-Carbolines via a Pd-Catalyzed Sequential Reaction from 2-Iodoanilines and $N$-Tosyl-enynamines. Org. Lett. 2012, 14, 38-41.<smiles>CCCCN1C(=O)[C@H](C(C)C)[C@H]([N+](=O)[O-])[C@H]1C(C)C</smiles>

1 of 21 examples $76 \%$ average yield, dr $3: 1$ to $30: 1$

Exercise \#87

Xiao-Xiao Li, Li-Li Zhu, Wen Zhou, and Zili Chen. Formal Intermolecular [2 + 2] Cycloaddition Reaction of Alleneamides with Alkenes via Gold Catalysis. Org. Lett. 2012, 14, 436-439. 

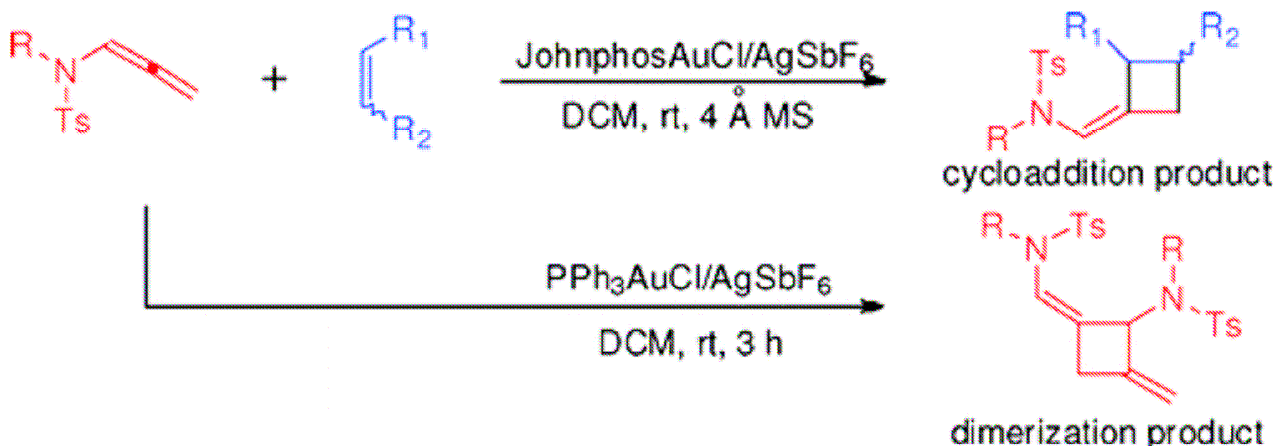

Exercise \#88

Lodi Mahendar, Jonnada Krishna, Alavala Gopi Krishna Reddy, Bokka Venkat Ramulu, and Gedu Satyanarayana. A Domino Palladium-Catalyzed $\mathrm{C}-\mathrm{C}$ and $\mathrm{C}-\mathrm{O}$ Bonds Formation via Dual O-H Bond Activation: Synthesis of 6,6-Dialkyl-6Hbenzo[c]chromenes. Org. Lett. 2012, 14, 628-631.<smiles>[R]c1cc(Br)c(C([R])([R])O)cc1[R]</smiles>

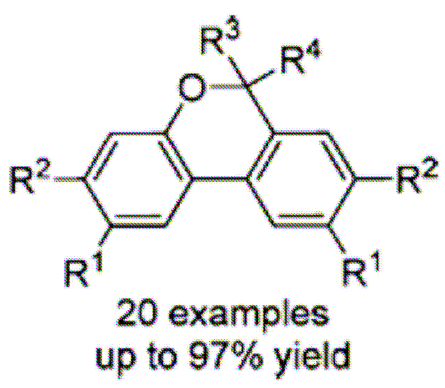

\section{Exercise \#89}

Lars Krogager Ransborg, Łukasz Albrecht, Christian F. Weise, Jesper R. Bak, and Karl Anker Jørgensen. Optically Active Thiophenes via an Organocatalytic One-Pot Methodology. Org. Lett. 2012, 14, 724-727.
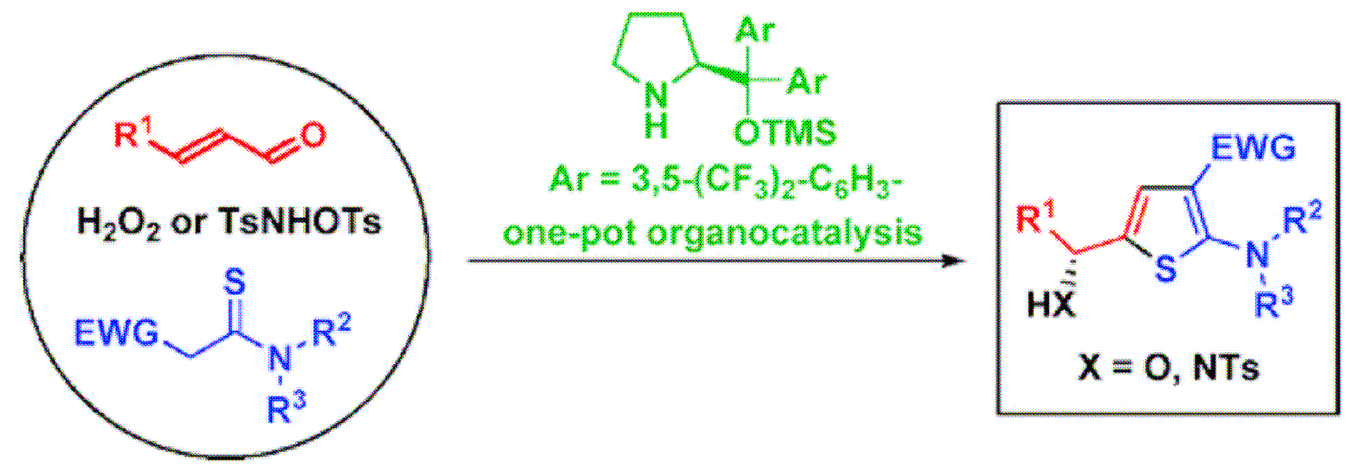

Exercise \#90

Guansai Liu, Phillip D. Wilkerson, Christopher A. Toth, and Hao Xu. Highly Enantioselective Cyclizations of Conjugated Trienes with Low Catalyst Loadings: Exercise \#91 
A Robust Chiral $\mathrm{N}$-Heterocyclic Carbene Enabled by Acetic Acid Cocatalyst. Org. Lett. 2012, 14, 858-861.

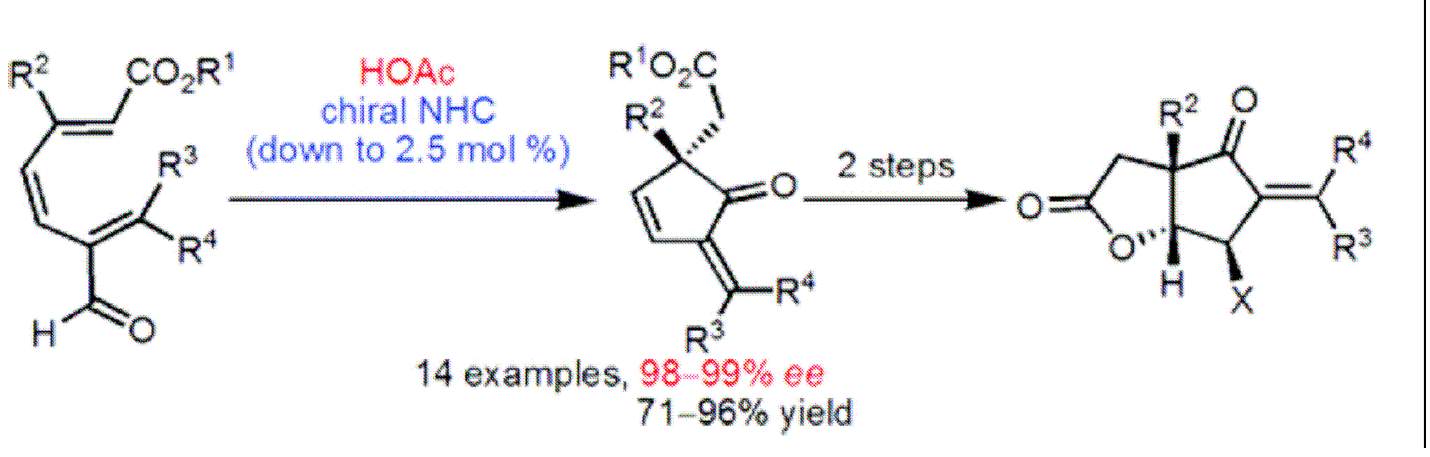

Exercise \#92

Christopher Kourra, Felix Klotter, Filippo Sladojevich, and Darren J. Dixon. Alkali Base-Initiated Michael Addition/Alkyne Carbocyclization Cascades. Org. Lett. 2012, 14, 1016-1019.<smiles>C#CCC(C(=O)OC)C(=O)N(Cc1ccccc1)C1CCC(=O)C=C1C</smiles>

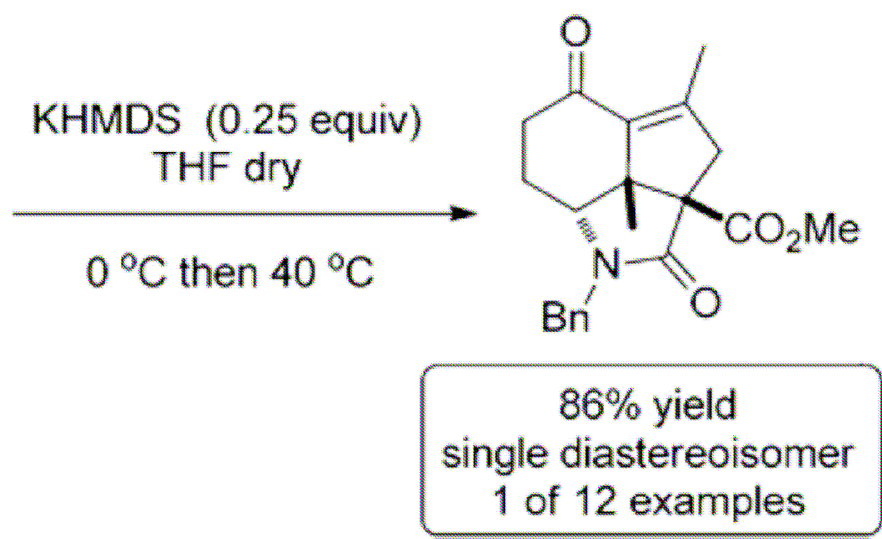

\section{Exercise \#93}

Morio Yonekawa, Yasuhito Koyama, Shigeki Kuwata, and Toshikazu Takata. Intramolecular 1,3-Dipolar Cycloaddition of Nitrile $\mathrm{N}$-Oxide Accompanied by Dearomatization. Org. Lett. 2012, 14, 1164-1167.<smiles>[R][R]1ccc(Oc2cccc([R])c2C#[N+][O-])cc1</smiles>

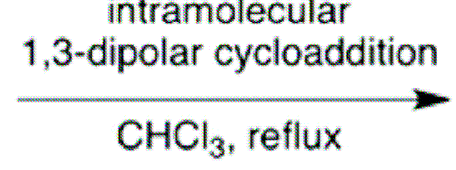<smiles>[R7]c1cccc2c1C1=NOC[C@@]13C(=CC[C@@H]3[R])O2</smiles> 


\section{Exercise \#94}

Fabio De Moliner and Christopher Hulme. Straightforward Assembly of

Phenylimidazoquinoxalines via a One-Pot Two-Step MCR Process. Org. Lett. 2012, 14, 1354-1357.

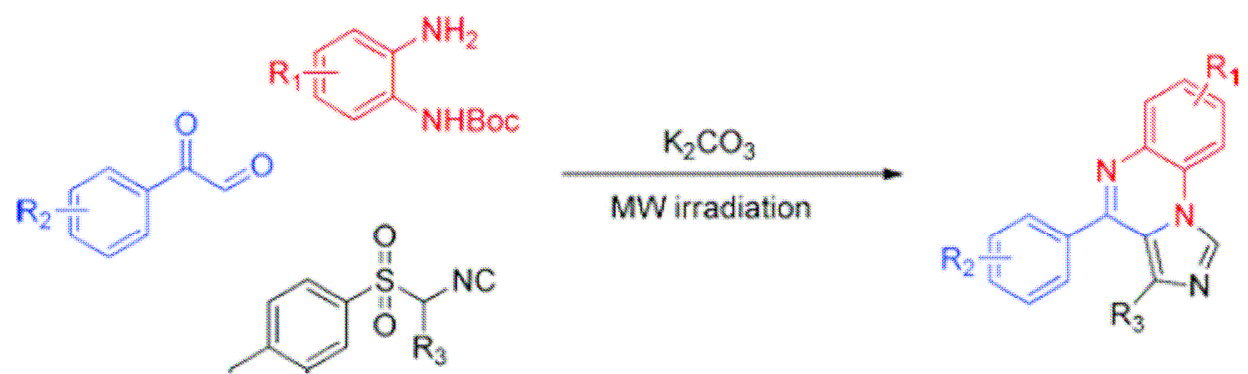

Exercise \#95

Min Jiang, Jian Li, Feng Wang, Yichao Zhao, Feng Zhao, Xiaochun Dong, and Weili Zhao. A Facile Copper-Catalyzed One-Pot Domino Synthesis of 5,12Dihydroindolo[2,1-b]quinazolines. Org. Lett. 2012, 14, 1420-1423.
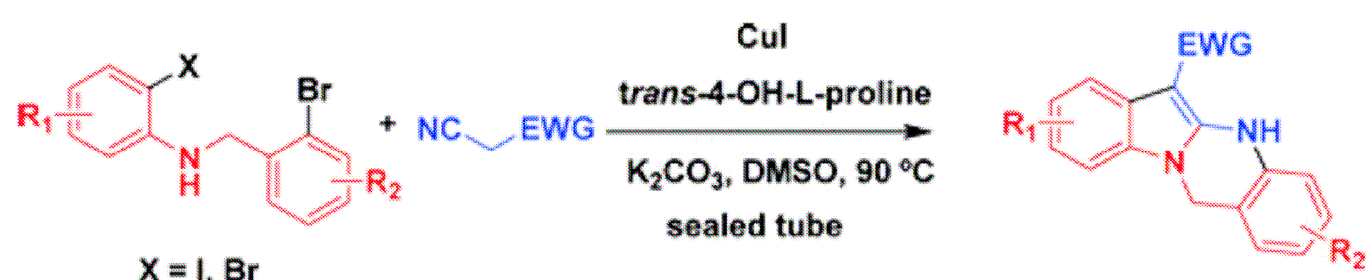

Exercise \#96

Noriyoshi Arai, Koichiro Tanaka, and Takeshi Ohkuma. Novel Intramolecular Photocyclization of $\alpha$-Arylthiophene Derivatives. Org. Lett. 2012, 14, 1488-1491.
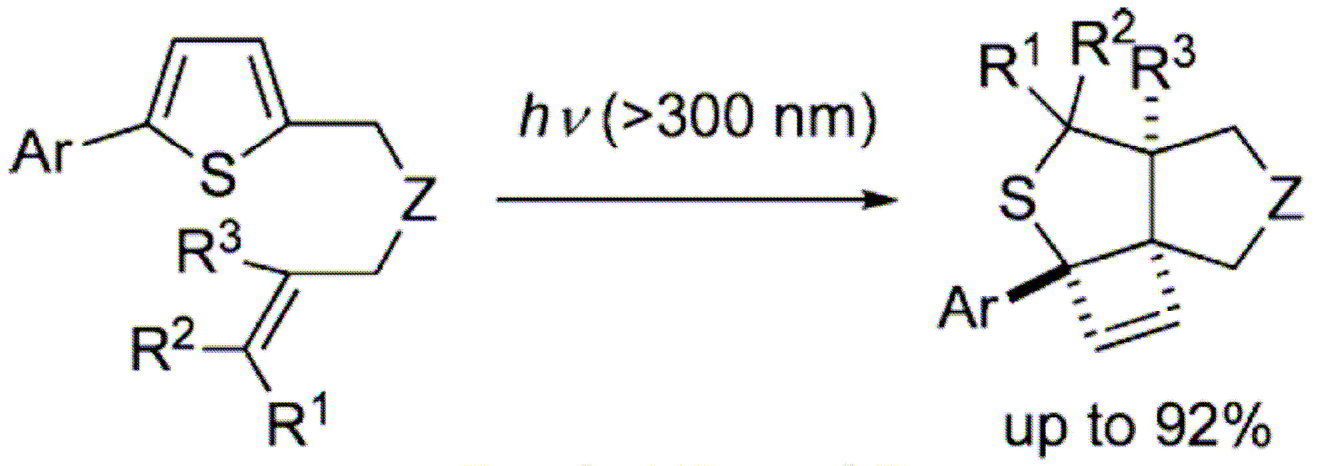

Exercise \#97

\section{$\mathrm{Z}=\mathrm{O}, \mathrm{NBOC}, \mathrm{CR}_{2}$}

Yong Luo and Jie Wu. Generation of Indeno[1,2-c]pyrroles via a Pd-Catalyzed Reaction of 2-Alkynylbromobenzene with Propargylic Sulfonamide. Org. Lett. 2012, 14, 15921595 . 

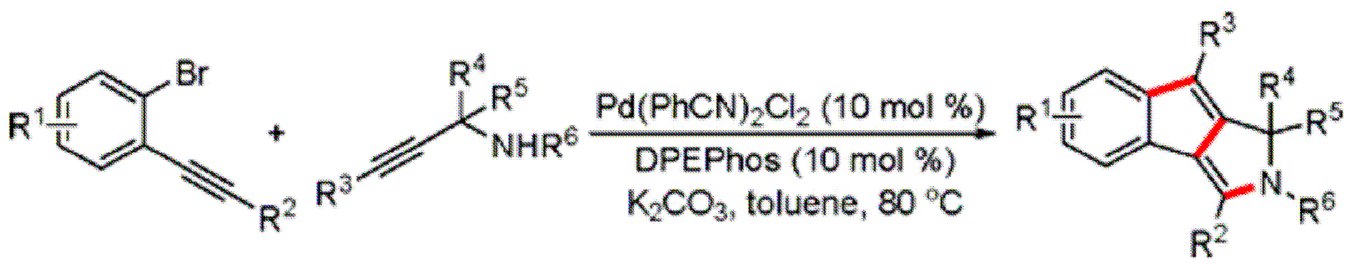

Exercise \#98

Jonathan D. Parrish, Michael A. Ischay, Zhan Lu, Song Guo, Noël R. Peters, and Tehshik P. Yoon. Endoperoxide Synthesis by Photocatalytic Aerobic $[2+2+2]$ Cycloadditions. Org. Lett. 2012, 14, 1640-1643.<smiles>COc1ccc(/C=C/COC/C=C/c2ccccc2)cc1</smiles><smiles>COc1ccc([C@@H]2OO[C@H](c3ccccc3)[C@@H]3COC[C@@H]32)cc1</smiles>

1,4-diols $\gamma$-hydroxyketones potential anticancer or antimalarial endoperoxides

\section{Exercise \#99}

Nozomi Saito, Taisuke Ichimaru, and Yoshihiro Sato. Total Synthesis of (-)-

Herbindoles A, B, and C via Transition-Metal-Catalyzed Intramolecular [2+2+2] Cyclization between Ynamide and Diynes. Org. Lett. 2012, 14, 1914-1917.
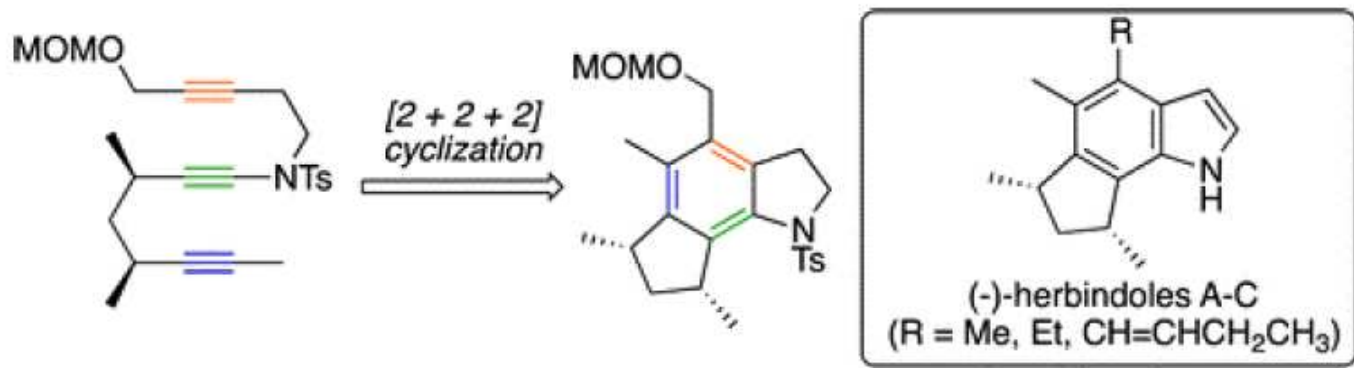

Exercise \#100

Arun K. Ghosh, Cuthbert D. Martyr, and Chun-Xiao Xu. Synthesis of Benzo-Fused Oxabicyclooctanes and Nonanes. Org. Lett. 2012, 14, 2002-2005. 


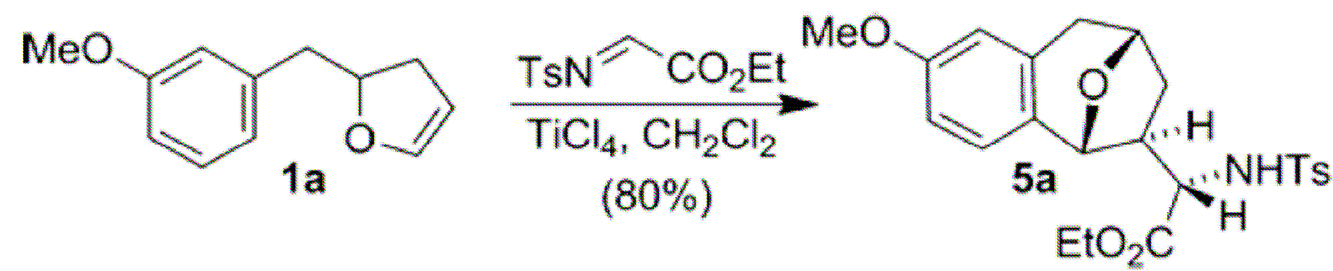

\section{Exercise \#101}

Takahiko Murata, Masahito Murai, Yuji Ikeda, Koji Miki, and Kouichi Ohe. Pd- and CuCatalyzed One-Pot Multicomponent Synthesis of Hetero $\alpha, \alpha$ '-Dimers of Heterocycles. Org. Lett. 2012, 14, 2296-2299.<smiles>[R]C([Y7])=C1CCCCC1C#C</smiles>

Exercise \#102
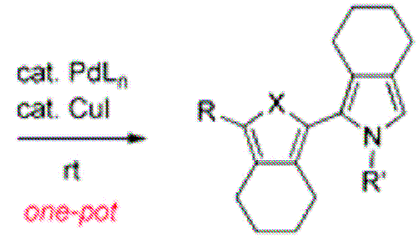

$\mathrm{X}=\mathrm{O}$ or $\mathrm{S}$

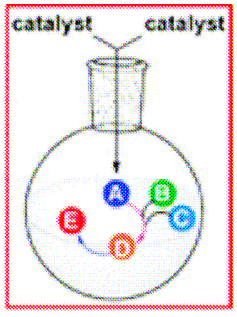

Bo Meng and Shengming Ma. Carbon-Carbon Bond Formation via the Electrophilic Addition of Carbocations to Allenes. Org. Lett. 2012, 14, 2674-2677.<smiles>[R]C1=C(C([R])Br)Cc2cc([R])ccc21</smiles><smiles>[R]C(=C)C([R])c1ccc([R])cc1</smiles>

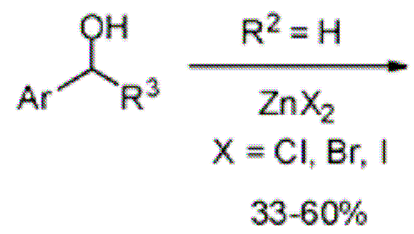<smiles>[X]CC(=Cc1ccc([R])cc1)C([R3])Br</smiles>

Exercise \#103

Long He, Mathieu Bekkaye, Pascal Retailleau, and Géraldine Masson. Chiral Phosphoric Acid Catalyzed Inverse-Electron-Demand Aza-Diels-Alder Reaction of Isoeugenol Derivatives. Org. Lett. 2012, 14, 3158-3161. 
<smiles>[R][R]c1ccc2c(c1)NC([R])[C@H](C)[C@H]2c1ccc(O)c(OC)c1</smiles>

Exercise \#104

Annamaria Martorana, Andrea Pace, Silvestre Buscemi, and Antonio Palumbo

Piccionello. Synthesis of Tetrasubstituted 4,4'-Biimidazoles. Org. Lett. 2012, 14, 32403243.<smiles>[R]C(O)[C@H](OC(=O)C(=O)c1nc([Al])[nH]c1-c1ccccc1)C(=O)O</smiles>

\section{Exercise \#105}

Ming Li, Han Cao, Yong Wang, Xiu-Liang Lv, and Li-Rong Wen. One-Pot Multicomponent Cascade Reaction of $N, S$-Ketene Acetal: Solvent-Free Synthesis of Imidazo[1,2-a]thiochromeno[3,2-e]pyridines. Org. Lett. 2012, 14, 3470-3473.

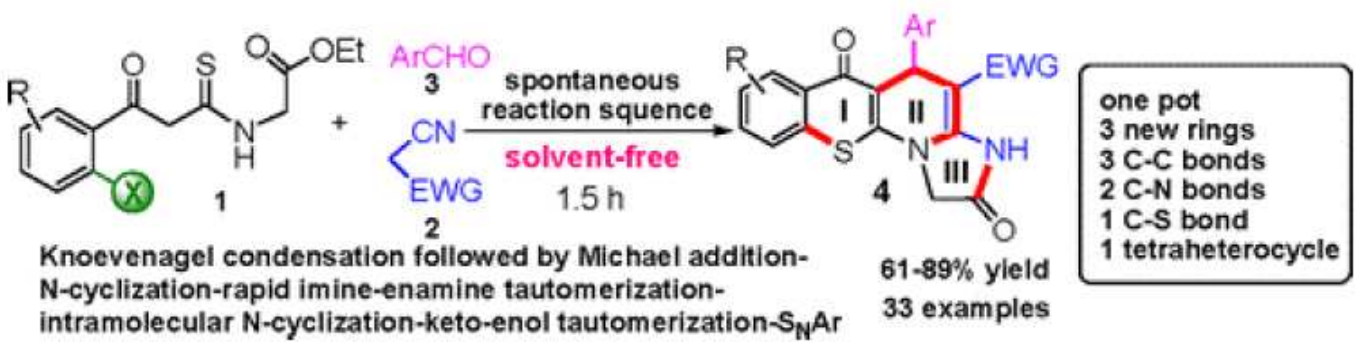

Exercise \#106

Dimitris Noutsias and Georgios Vassilikogiannakis. First Total Synthesis of

Paracaseolide A. Org. Lett. 2012, 14, 3565-3567. 


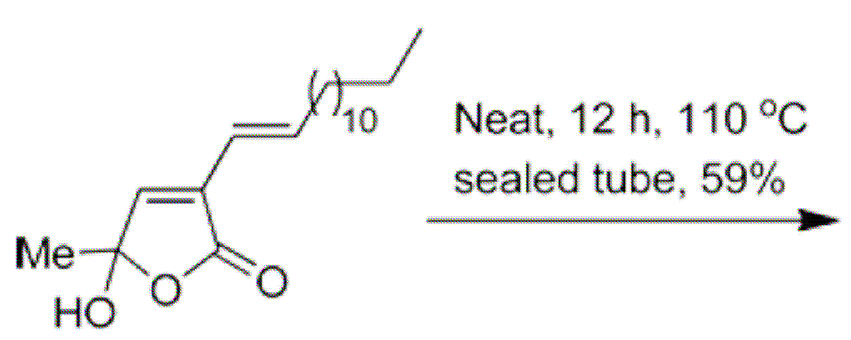

Exercise \#107

Zhiyuan Chen, Mengjing Zeng, Jianjun Yuan, Qin Yang, and Yiyuan Peng. Novel Silver Tetrafluoroborate Catalyzed Electrophilic Cascade Cyclization Reaction: A Facile Approach to the Synthesis of Halo-Substituted Benzo[a]fluorenols. Org. Lett. 2012, 14, 3588-3591.<smiles>[R][R4]1ccc(C#Cc2ccc([R])c(C#CC(O)c3ccccc3[R])c2)cc1</smiles><smiles>[R]c1ccc(C2(O)c3cc[R1]([H])cc3-c3c([X])cc4ccccc4c32)cc1</smiles>

\section{Exercise \#108}

Kenichi Ogata, Itsuki Ohashi, and Shin-ichi Fukuzawa. Rhodium-Catalyzed ThreeComponent Reaction between Silylacetylene and Two Ketenes Leading to 1,3-Enynes Bearing a Carboxylic Ester Group via Double Insertion of Ketenes. Org. Lett. 2012, 14, 4214-4217.

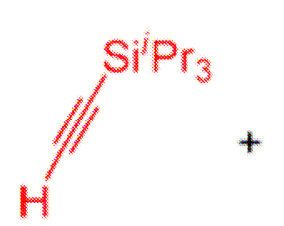<smiles>[R]C([R])=C=O</smiles>

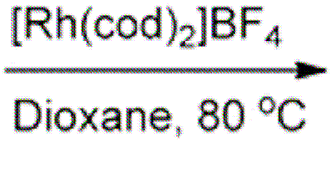<smiles>[R]C([R])=C(C#C[SnH3])OC(=O)C([R])([R])[2H]</smiles>

Double insertion of ketene to alkyne $\mathrm{C}-\mathrm{H}$ bond

\section{Exercise \#109}

Krishnamoorthy Muralirajan, Kanniyappan Parthasarathy, and Chien-Hong Cheng. Ru(II)Catalyzed Amidation of 2-Arylpyridines with Isocyanates via C-H Activation. Org. Lett. 2012, 14, 4262-4265. 


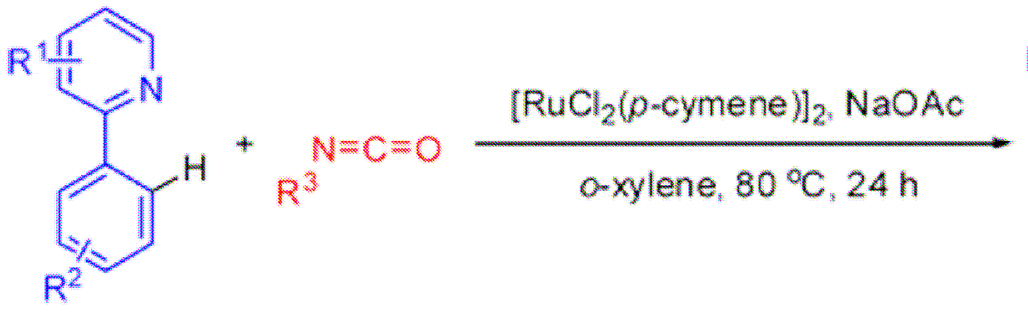<smiles>[R]NC(=O)c1cc[R]([H])cc1-c1c[R1]ccn1</smiles>

Exercise \#110

Takashi Aoki, Shunsuke Koya, Ryu Yamasaki, and Shinichi Saito. Cycloaddition Reaction of 2-Vinylazetidines with Benzyne: A Facile Access to 1-Benzazocine Derivatives. Org. Lett. 2012, 14, 4506-4509.

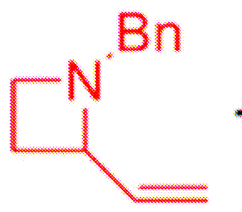<smiles>c1ccccc1</smiles>
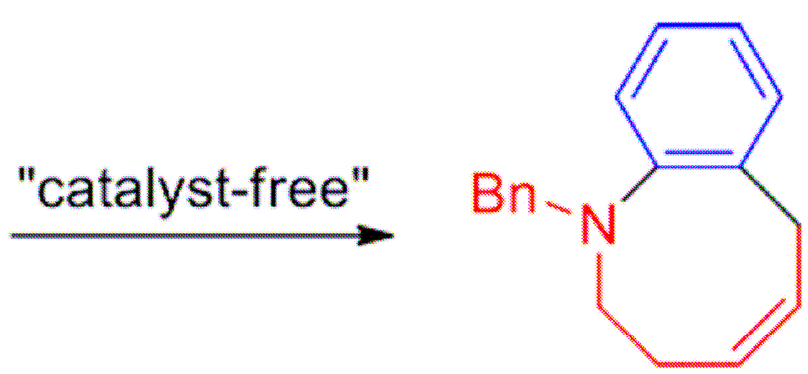

Exercise \#111

Huiyuan Wang, Lili Li, Wei Lin, Pan Xu, Zhibin Huang, and Daqing Shi. An Efficient Synthesis of Pyrrolo[2,3,4-kl]acridin-1-one Derivatives Catalyzed by Lproline. Org. Lett. 2012, $14,4598-4601$.<smiles>[R]NC1=CC(=O)CC([R])([R])C1</smiles>

Exercise \#112

Wen-Juan Hao, Xiao-Ping Xu, Hui-Wen Bai, Shun-Yi Wang, and Shun-Jun Ji. Efficient Multicomponent Strategy to Pentacyclic Pyrazole-Fused Naphtho[1,8- $f g]$ isoquinolines through Cleavage of Two Carbon-Carbon Bonds. Org. Lett. 2012, 14, 4894-4897. 
<smiles>[R]n1nc(F)cc1N</smiles>

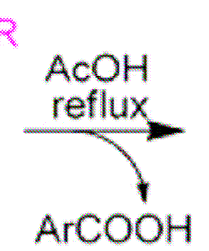<smiles>[R]c1nn([R1])c2c3c4c5cccc(cccc-4c(=O)c3=C(Br)N=2)c15</smiles>

Exercise \#113

Quan Cai, Xiao-Wei Liang, Shou-Guo Wang, Jun-Wei Zhang, Xiao Zhang, and Shu-Li You. Ring-Closing Metathesis/Isomerization/Pictet-Spengler Cascade via Ruthenium/Chiral Phosphoric Acid Sequential Catalysis. Org. Lett. 2012, 14, 5022 5025 .

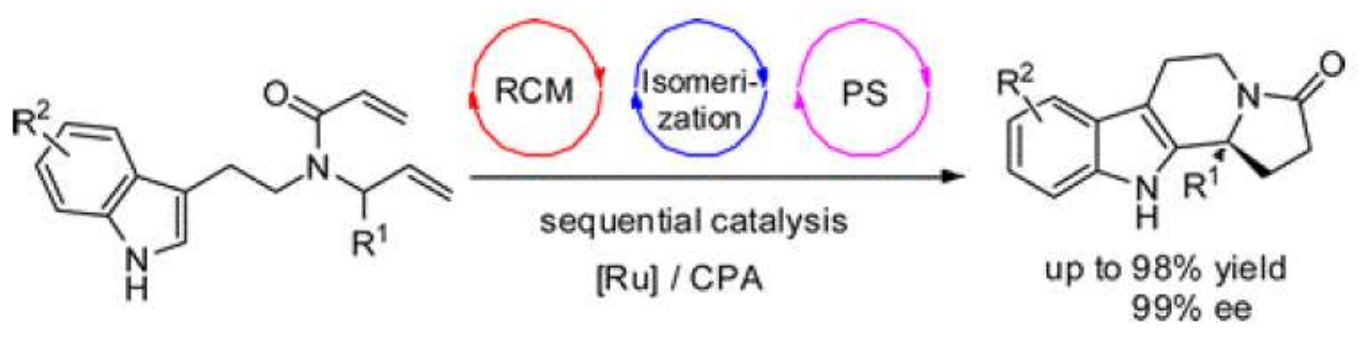

Exercise \#114

Zhengwang Chen, Wei Zeng, Huanfeng Jiang, and Liangxian Liu. Cu(II)-Catalyzed Synthesis of Naphthalene-1,3-diamine Derivatives from Haloalkynes and Amines. Org. Lett. 2012, 14, 5385-5387.<smiles>[Y][R19]=[W]</smiles>

\section{Exercise \#115}

Santosh J. Gharpure, P. Niranjana, and Suheel K. Porwal. Stereoselective Synthesis of Oxa- and Aza-Angular Triquinanes Using Tandem Radical Cyclization to Vinylogous Carbonates and Carbamates. Org. Lett. 2012, 14, 5476-5479. 


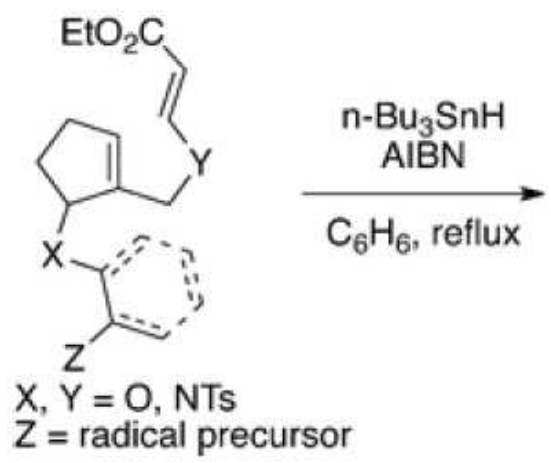

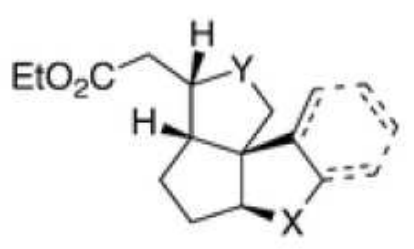

$45-82 \%$

$\mathrm{dr} \geq 19: 1$

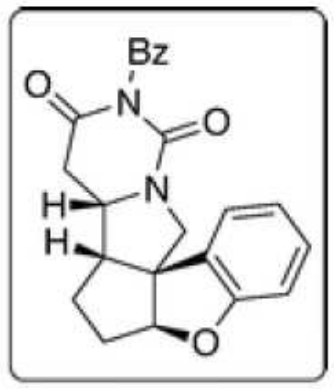

Exercise \#116

Jian Hu, Wei Dong, Xin-Yan Wu, and Xiaofeng Tong. PPh3-Catalyzed (3+3)

Annulations of 5-Acetoxypenta-2,3-dienoate with 1C,3OBisnucleophiles: Facile Entry to Stable Monocyclic 2H-Pyrans. Org. Lett. 2012, 14, 5530-5533.
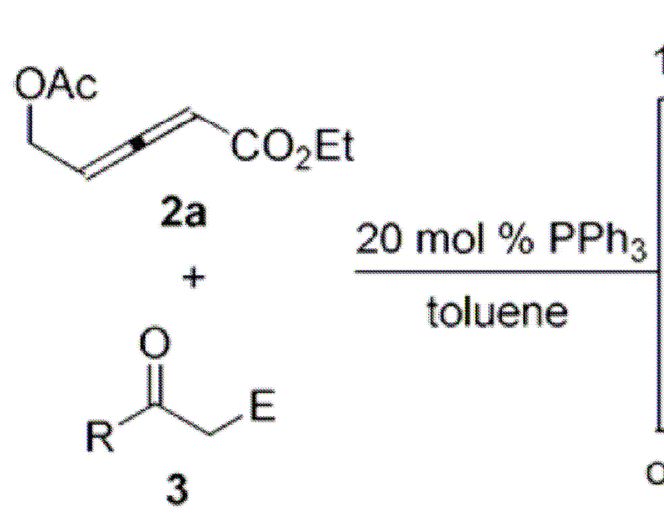

\section{Exercise \#117}

1.2 equiv $\mathrm{NaOH}$<smiles>[R]c1oc(CC)c(C(=O)OCC)c1F</smiles>

1.0 equiv $\mathrm{AcOH}$ or without additve rt<smiles>[R]C1=C(F)C(C(=O)OCC)=CC(C)O1</smiles>

Wei Yuan, Xiang Dong, Min Shi, Patrick McDowell, and Guigen Li. Rh(I)-catalyzed Pauson-Khand-type Cycloaddition Reaction of Enevinylidenecyclopropanes with Carbon Monoxide (CO). Org. Lett. 2012, 14, 5582-5585.<smiles>[X]CC=C=C=C1CC1</smiles>

$$
\mathrm{X}=\mathrm{N}, \mathrm{O}, \mathrm{C}
$$

$\left[\mathrm{Rh}(\mathrm{COD}) \mathrm{Cl}_{2}(2 \mathrm{~mol} \%)\right.$ TCE, CO (1 atm), $80^{\circ} \mathrm{C}, 1 \mathrm{~h}$ 13 examples<smiles>[X]CC1=C2C(=C([R])C1)CC(=O)C21CC1</smiles>

up to $99 \%$ yield 
Exercise \#118

Yuta Miyauchi, Keiichi Noguchi, and Ken Tanaka. Rhodium-Catalyzed One-Pot Intermolecular [2 + 2 + 2] Trimerization/Asymmetric Intramolecular [4 + 2]

Cycloaddition of Two Aryl Ethynyl Ethers and 5-Alkynals. Org. Lett. 2012, 14, 58565859.

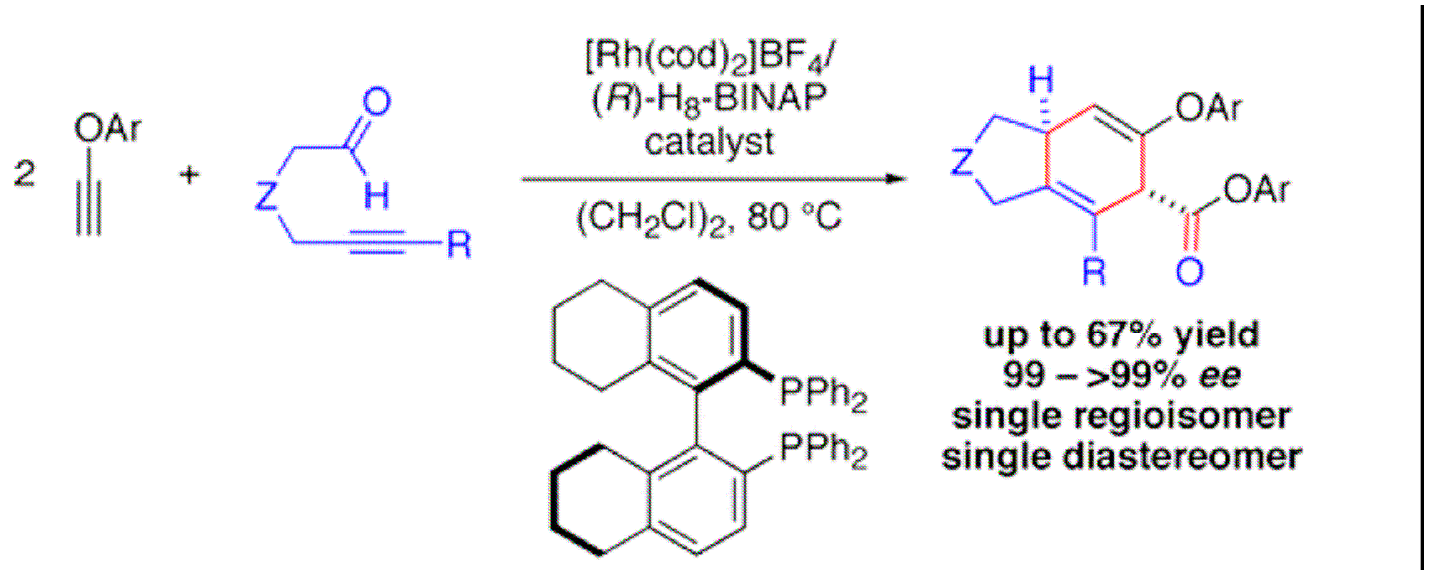

Exercise \#119

Falko Abels, Chris Lindemann, Eva Koch, and Christoph Schneider. A General Organocatalytic Approach toward the Enantioselective Total Synthesis of Indolizidine Based Alkaloids. Org. Lett. 2012, 14, 5972-5975.

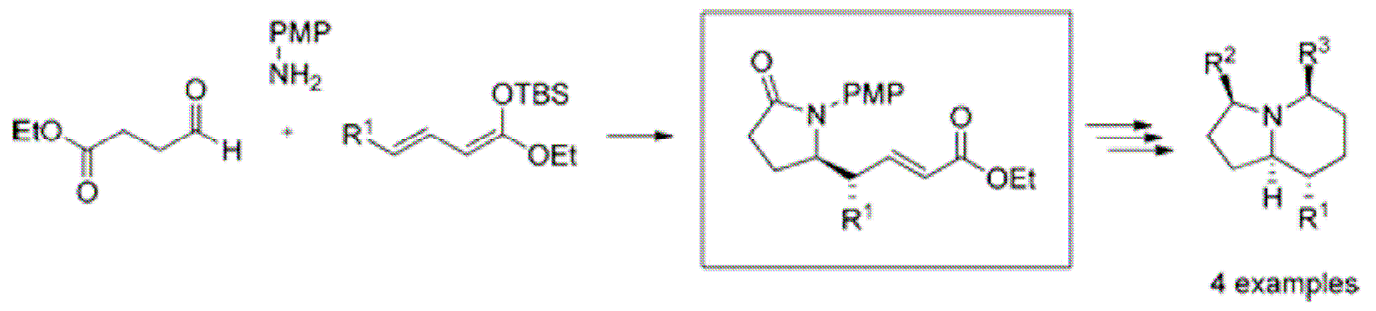

Exercise \#120

Christine Meck, Noushad Mohd, and Ryan P. Murelli. An Oxidopyrylium

Cyclization/Ring-Opening Route to Polysubstituted $\alpha$-Hydroxytropolones. Org. Lett. 2012, 14, 5988-5991.<smiles>COc1cc([Ga])[o+]cc1O</smiles><smiles>[R]#CPCCP</smiles><smiles>[R7]c1cc(O)c(=O)c(O)c([R])c1[13CH2][13CH3]</smiles>

Exercise \#121 
Ying-Chieh Wong, Chen-Tso Tseng, Tzu-Ting Kao, Yu-Cheng Yeh, and Kak-Shan Shia. Tandem Cyclization of $\alpha$-Cyano $\alpha$-Alkynyl Aryl Ketones Induced by tert-Butyl Hydroperoxide and Tetrabutylammonium Iodide. Org. Lett. 2012, 14, 6024-6027.<smiles>[R]C#CCCC([R])([R])C(C#N)C(=O)c1c#[R1]ccc1</smiles>

$\mathrm{R}=\mathrm{TMS}, \mathrm{Ph}, 4-\mathrm{CO}_{2} \mathrm{MePh}$, $4-\mathrm{OMePh}$
TBHP (1.1 equiv) TBAI (20 mol \%), PhH $0.03 \mathrm{M}, 80^{\circ} \mathrm{C}, 0.5 \sim 1 \mathrm{~h}$

$\mathrm{R}^{1}$<smiles>[R]C1=C2CCC([R])([R])C2(C=O)C(=O)C2=C1C=C[Y1]C=C2</smiles>

15 examples

$72 \sim 98 \%$

Exercise \#122

Rong Zhou, Jianfang Wang, Chong Duan, and Zhengjie He. Phosphine-Triggered Tandem Annulation between Morita-Baylis-Hillman Carbonates and Dinucleophiles: Facile Syntheses of Oxazepanes, Thiazepanes, and Diazepanes. Org. Lett. 2012, 14, 6134-6137.

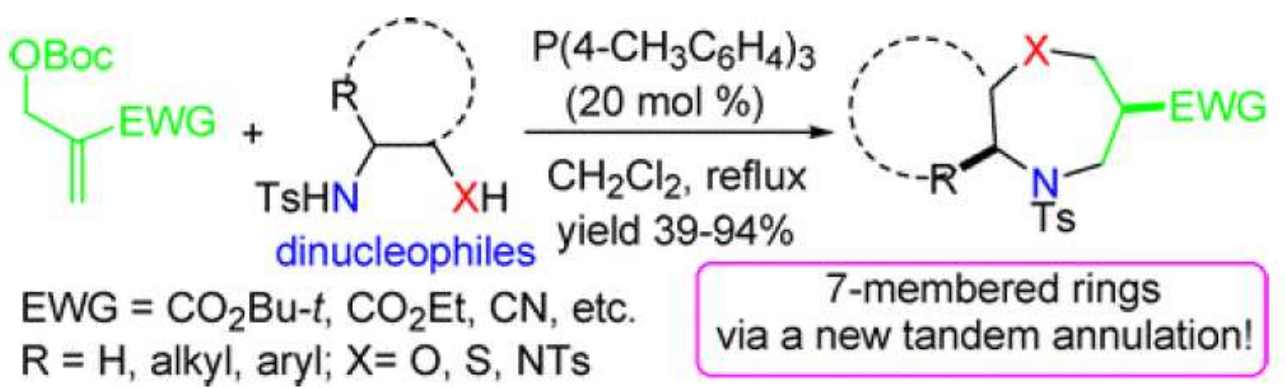

Exercise \#123

Li-Jin Dong, Tian-Tian Fan, Chao Wang, and Jian Sun. One-Pot Formation of Chiral Polysubstituted 3,4-Dihydropyrans via a Novel Organocatalytic Domino Sequence Involving Alkynal Self-Condensation. Org. Lett. 2013, 15, 204-207.

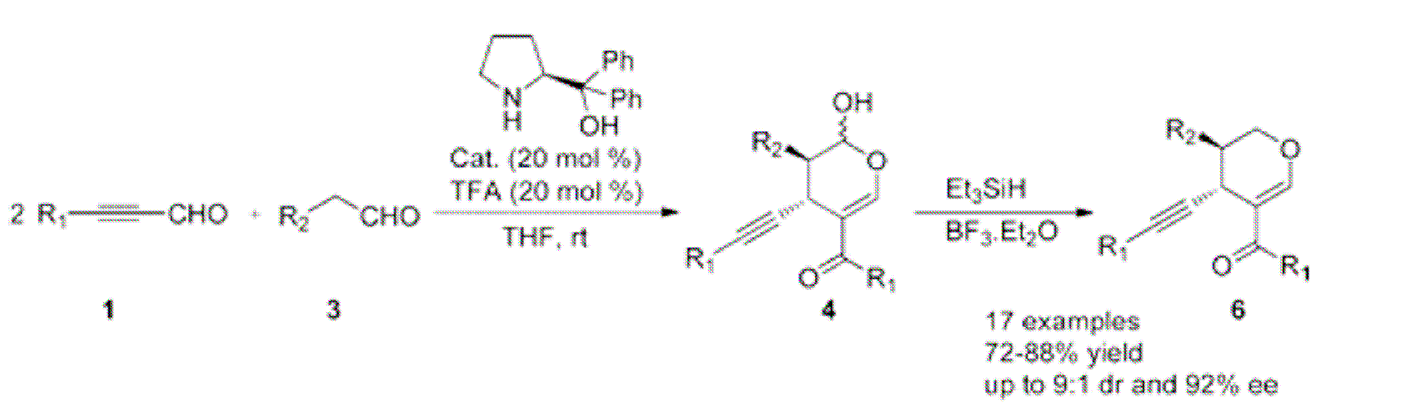


Yan-ping Zhu, Zhuan Fei, Mei-cai Liu, Feng-cheng Jia, and An-xin Wu. Direct One-Pot Synthesis of Luotonin F and Analogues via Rational Logical Design. Org. Lett. 2013, $15,378-381$.

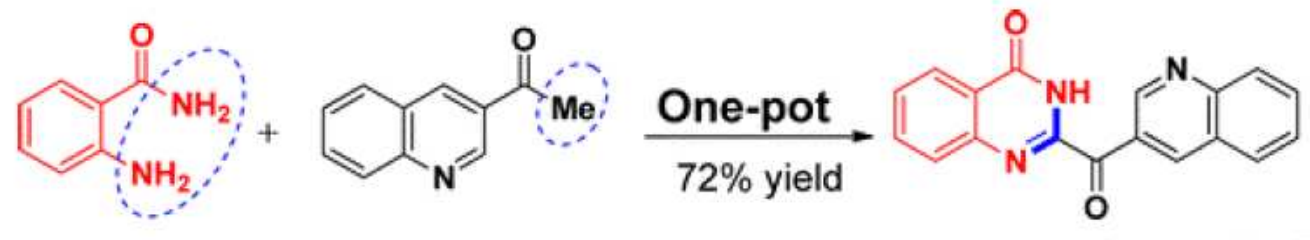

\section{luotonin F}

\section{Exercise \#125}

Wen-Ming Shu, Yan Yang, Dong-Xue Zhang, Liu-Ming Wu, Yan-Ping Zhu, Guo-Dong Yin, and An-Xin Wu. Highly Efficient Synthesis of 3a,6a-Dihydrofuro[2,3- $b$ ]furans via a Novel Bicyclization. Org. Lett. 2013, 15, 456-459.<smiles>[R]C(=O)C=C([R])C([R])=O</smiles>

$\mathrm{R}^{1}=($ Hetero $)$ aryl

$\mathrm{R}^{2}=($ Hetero) aryl, alkyl

$\mathrm{R}^{3}=\mathrm{CO}_{2} \mathrm{Et}, \mathrm{CN}, \mathrm{COPh}, \mathrm{COCH}_{3}$

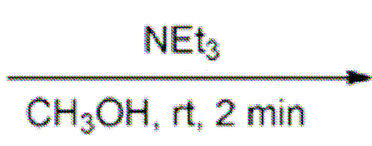

up to $96 \%$ yield

Exercise \#126

Hua Wu, Yu-Ping He, and Liu-Zhu Gong. Direct Access to Enantioenriched Spiroacetals through Asymmetric Relay Catalytic Three-Component Reaction. Org. Lett. 2013, 15, 460-463.
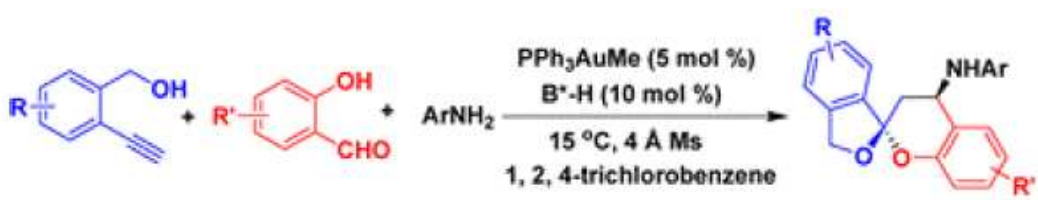

up to $97 \%$ yield $>25: 1 \mathrm{dr}, 95 \%$ ee<smiles>[R]C1=C([R])C2C(C#N)=C(N)OC2([R])O1</smiles>

\section{2 examples}

Exercise \#127

Leoni I. Palmer and Javier Read de Alaniz. Rapid and Stereoselective Synthesis of Spirocyclic Ethers via the Intramolecular Piancatelli Rearrangement. Org. Lett. 2013, 15, 476-479. 
<smiles>[R]C(O)c1ccc(CCCO)o1</smiles><smiles>[R]C1C(=O)C=C[C@]12CCCO2</smiles>

\section{7 examples} trans selective

Exercise \#128

Qunsheng Guo and John Cong-Gui Zhao. Highly Enantioselective Three-Component Direct Mannich Reactions of Unfunctionalized Ketones Catalyzed by Bifunctional Organocatalysts. Org. Lett. 2013, 15, 508-511.

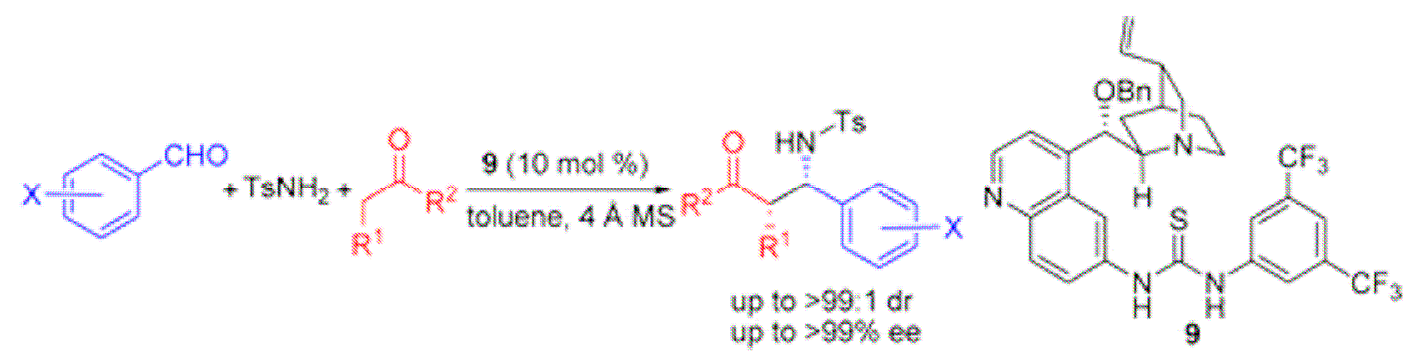

Exercise \#129

Szymon Kłossowski, Barbara Wiraszka, Stanisław Berłożecki, and Ryszard Ostaszewski. Model Studies on the First Enzyme-Catalyzed Ugi Reaction. Org. Lett. 2013, 15, 566569.<smiles>C[O+]=CCC(C)C</smiles><smiles>NCC12CCCCCC1CCC2</smiles><smiles>CCOC(=O)C[N+](=O)[O-]</smiles><smiles></smiles><smiles>CC(C)CC(NCCCNC(=O)CNCc1ccccc1)C(=O)O</smiles>

Exercise \#130

Nicolas Kern, Marie Hoffmann, Aurélien Blanc, Jean-Marc Weibel, and Patrick Pale. Gold(I)-Catalyzed Rearrangement of $N$-Aryl 2-Alkynylazetidines to Pyrrolo[1,2a]indoles. Org. Lett. 2013, 15, 836-839. 
<smiles>[R]C#CC1C([R])CN1c1[R]ccc1</smiles>

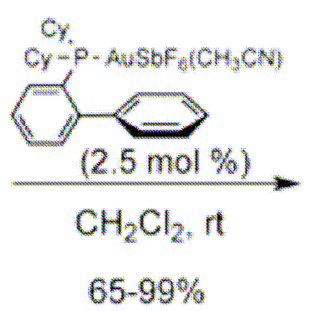

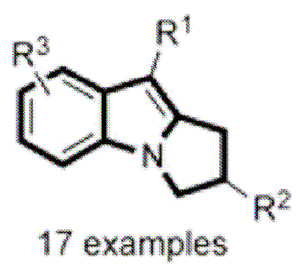

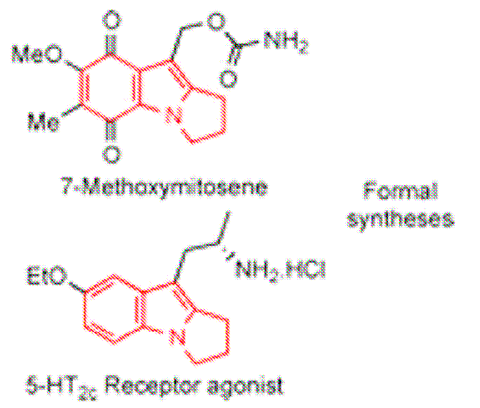

Exercise \#131

Shengkun Li, Kexuan Huang, Jiwen Zhang, Wenjun Wu, and Xumu Zhang. Cascade Synthesis of Fenpiprane and Related Pharmaceuticals via Rhodium-Catalyzed Hydroaminomethylation. Org. Lett. 2013, 15, 1036-1039.

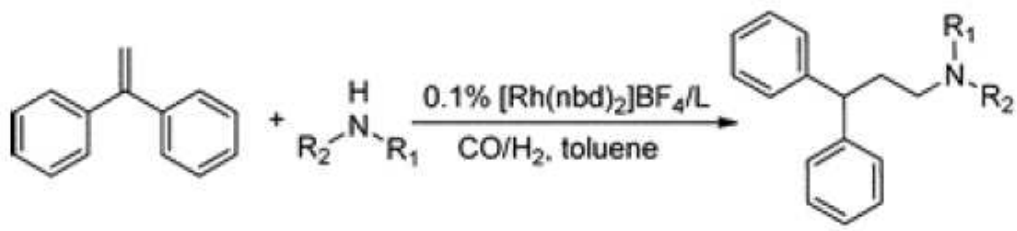<smiles>N#Cc1cccc2c(Cc3ccccc3)c(Cc3ccccc3)c(Cc3ccccc3)cc12</smiles>

Exercise \#132

Johannes L. Vrijdag, An M. Van den Bogaert, and Wim M. De Borggraeve. Scaffold Hopping via a Transannular Rearrangement-Encompassing Cascade. Org. Lett. 2013, $15,1052-1055$.<smiles>[R]N1C(=O)CNC(=O)c2ccccc21</smiles><smiles>[R]C(=O)OC([R2])C</smiles><smiles>[R]c1nc2c(=O)n([R7])c3ccccc3c2o1</smiles>

12 examples up to $98 \%$ yield

Exercise \#133

Hua Cao, Haiying Zhan, Jinghe Cen, Jingxin Lin, Yuanguang Lin, Qiuxia Zhu, Minling $\mathrm{Fu}$, and Huanfeng Jiang. Copper-Catalyzed C-O Bond Formation: An Efficient One-Pot Highly Regioselective Synthesis of Furans from (2-Furyl)Carbene Complexes. Org. Lett. 2013, 15, 1080-1083. 


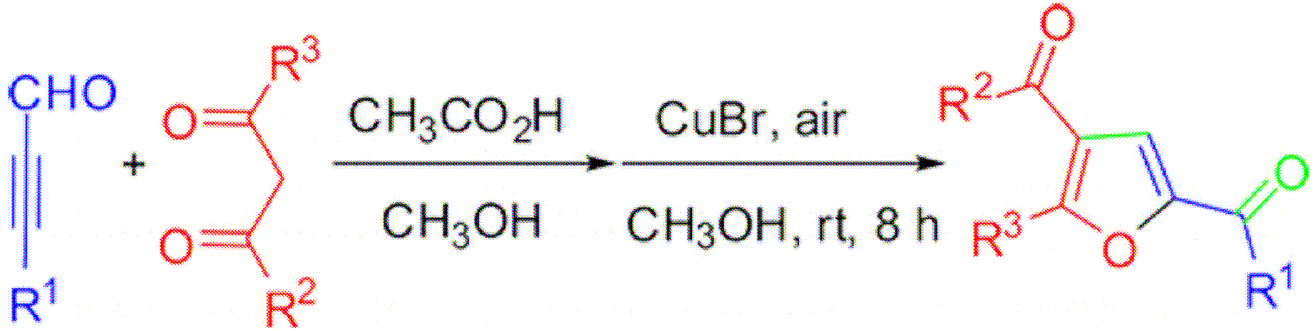

Exercise \#134

Juliane Keilitz, Stephen G. Newman, and Mark Lautens. Enantioselective Rh-Catalyzed Domino Transformations of Alkynylcyclohexadienones with Organoboron Reagents.

Org. Lett. 2013, 15, 1148-1151.<smiles>C#CCOC1(C)C=CC(=O)C=C1</smiles><smiles>[R]c1ccc(Br)cc1</smiles>
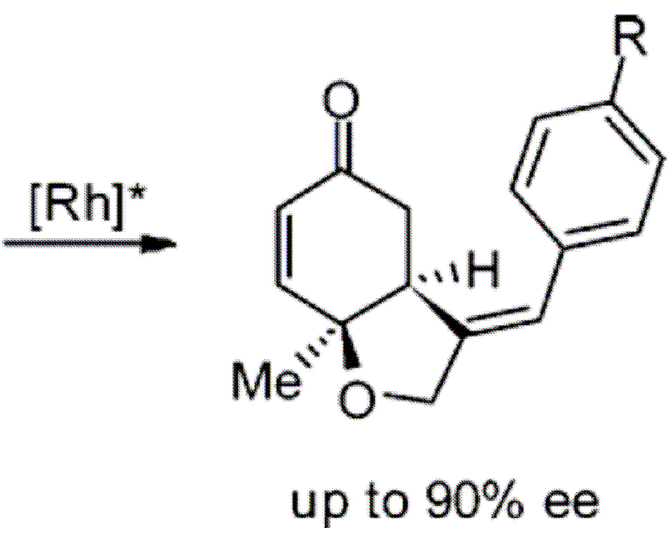

Exercise \#135

Ming Li, Xiu-Liang Lv, Li-Rong Wen, and Zhi-Qiang Hu. Direct Solvent-Free Regioselective Construction of Pyrrolo[1,2-a][1,10]phenanthrolines Based on Isocyanide-Based Multicomponent Reactions. Org. Lett. 2013, 15, 1262-1265.
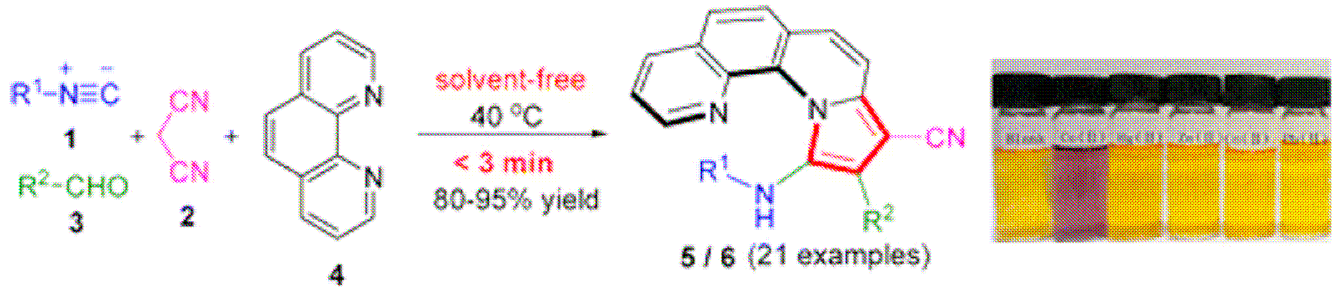

Exercise \#136

Stephan Rigol, Lothar Beyer, Lothar Hennig, Joachim Sieler, and Athanassios Giannis. Hünlich Base: (Re)Discovery, Synthesis, and Structure Elucidation after a Century. Org. Lett. 2013, 15, 1418-1420. 
<smiles>Cc1ccc(N)cc1N</smiles><smiles>Cc1cc2c(cc1N)N1Cc3cc(C)c(N)cc3N(C2)[C@@H](O)C1</smiles>

Exercise \#137

Xiaocong Xie, Yi Li, and Joseph M. Fox. Selective Syntheses of $\Delta \alpha, \beta$ and $\Delta \beta, \gamma$ Butenolides from Allylic Cyclopropenecarboxylates via Tandem Ring Expansion/[3,3]Sigmatropic Rearrangements. Org. Lett. 2013, 15, 1500-1503.

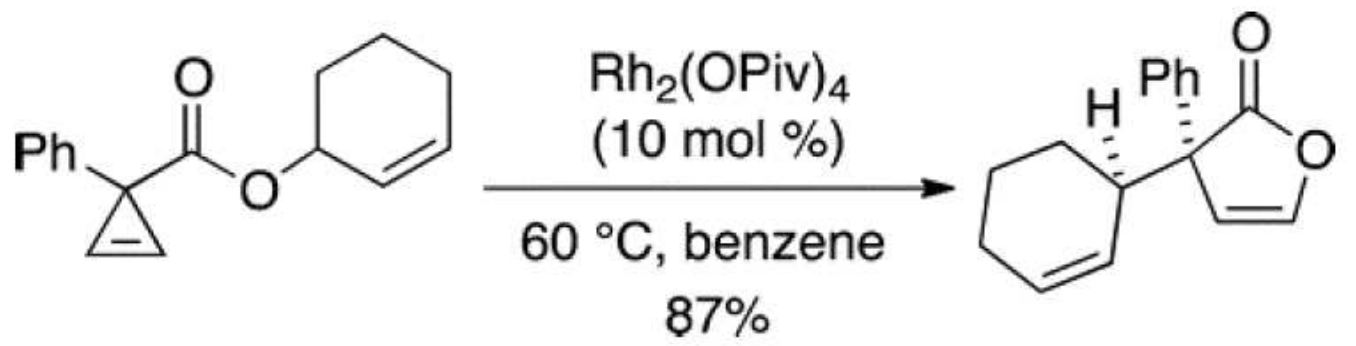

Exercise \#138

Yu Qian, Peter J. Zavalij, Wenhao Hu, and Michael P. Doyle. Bicyclic Pyrazolidinone Derivatives from Diastereoselective Catalytic [3 + 3]-Cycloaddition Reactions of Enoldiazoacetates with Azomethine Imines. Org. Lett. 2013, 15, 1564-1567.

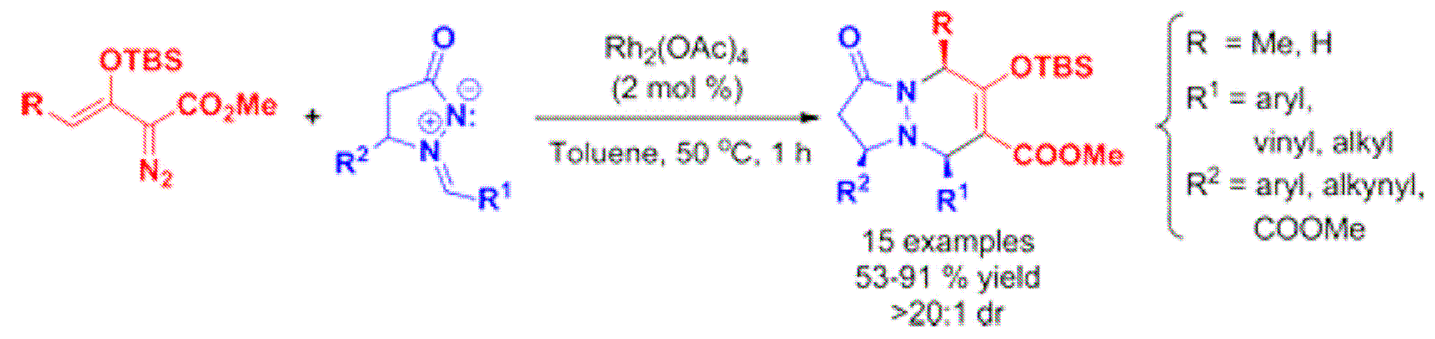

Exercise \#139

Amit Kumar, Zhenghua Li, Sunil K. Sharma, Virinder S. Parmar, and Erik V. Van der Eycken. An Expedient Route to Imidazo[1,4]diazepin-7-ones via A Post-Ugi GoldCatalyzed Heteroannulation. Org. Lett. 2013, 15, 1874-1877.<smiles>[R]C#CC(=O)O</smiles>

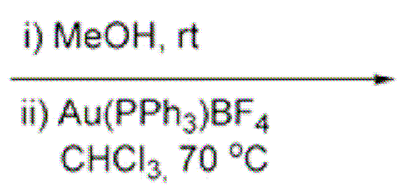<smiles>[R5]NC(=O)C1c2c([R])nc([R16])n2C([R])=CC(=O)N1[R]</smiles> 
Exercise \#140

Xiang Wang, Shun-Yi Wang, and Shun-Jun Ji. Isocyanide-Based Multicomponent Reactions: Catalyst-Free Stereoselective Construction of Polycyclic Spiroindolines. Org. Lett. 2013, 15, 1954-1957.

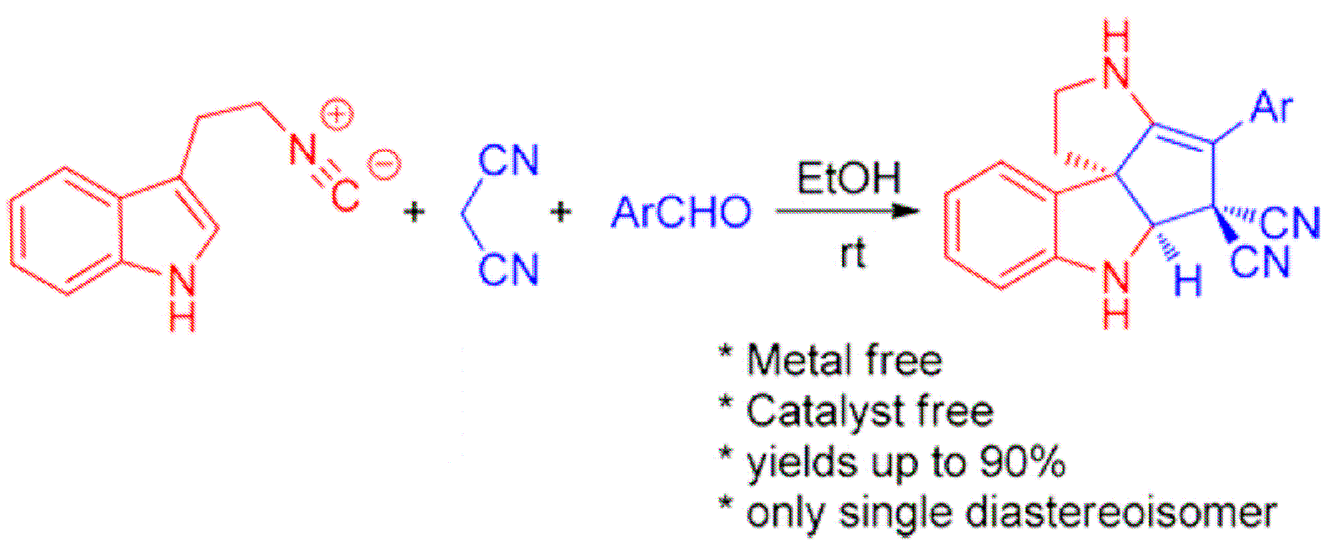

Exercise \#141

Lei Zhang, Lorenzo Sonaglia, Jason Stacey, and Mark Lautens. Multicomponent Multicatalyst Reactions (MC)2R: One-Pot Synthesis of 3,4-Dihydroquinolinones. Org. Lett. 2013, 15, 2128-2131.

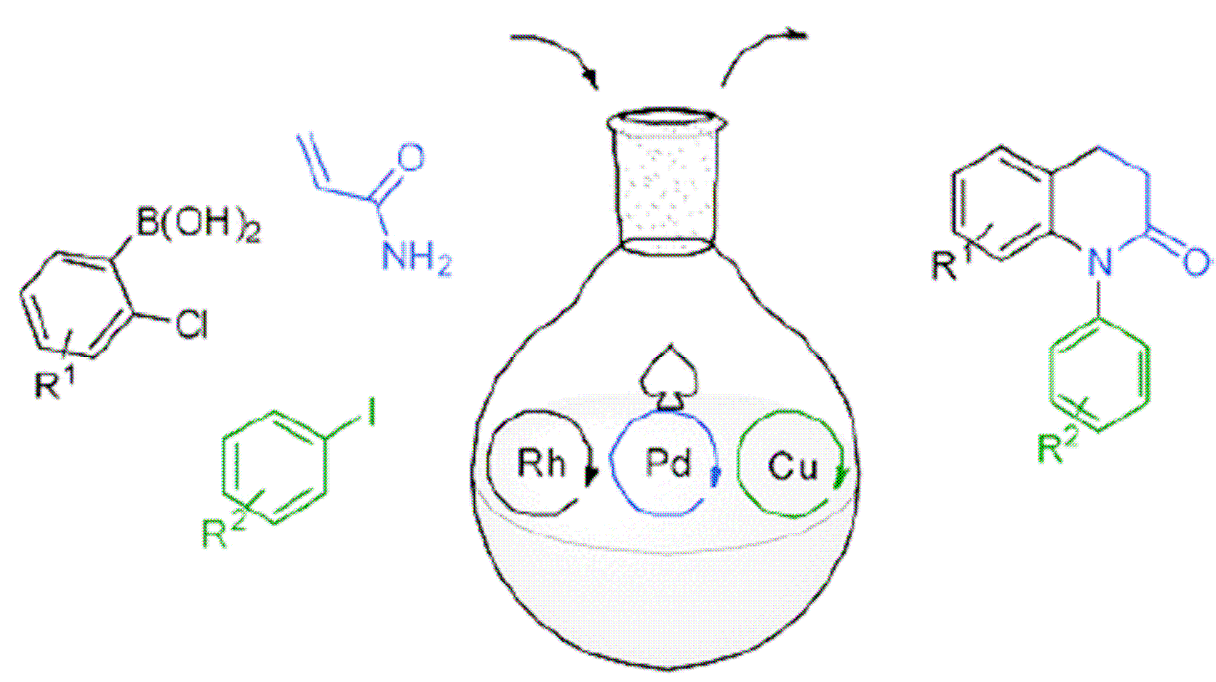

Exercise \#142

Boris A. Trofimov, Ludmila V. Andriyankova, Lina P. Nikitina, Kseniya V. Belyaeva, Anastasia G. Mal'kina, Lubov N. Sobenina, Andrei V. Afonin, and Igor A. Ushakov. Stereoselective Tandem Ring Opening of Imidazoles with Electron-Deficient Acetylenes and Water: Synthesis of Functionalized (Z,Z)-1,4-Diaza-2,5-dienes. Org. Lett. 2013, 15, 2322-2324. 

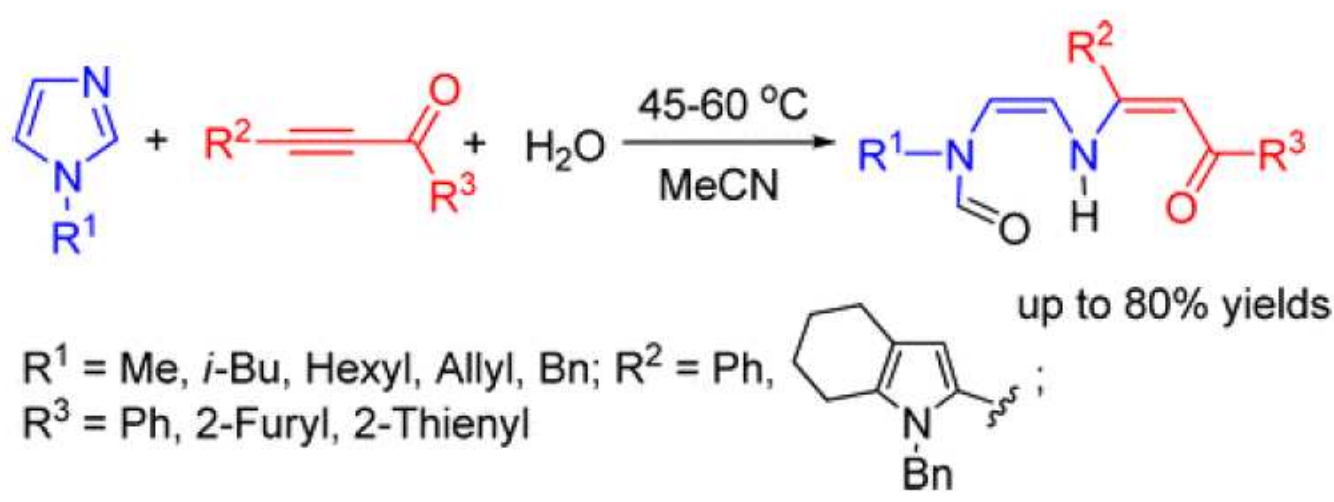

Exercise \#143

Ben Bradshaw, Claudio Parra, and Josep Bonjoch. Organocatalyzed Asymmetric Synthesis of Morphans. Org. Lett. 2013, 15, 2458-2461.<smiles>[R]CC(=O)CC(=O)OCCCC</smiles><smiles>OC(O)C(O)(c1ccccc1)C1CCCN1</smiles><smiles>[R]C1C[C@@H]([2H])[C@@H]([2H])C(C(=O)OC(C)(C)C)=C1O</smiles>

A tandem/domino one-pot bis-cyclization

Exercise \#144

Aiguo Song, Xiaobei Chen, Xixi Song, Xinshuai Zhang, Shilei Zhang, and Wei Wang. Synthesis of Benzoxazoles via an Amine-Catalyzed [4+1] Annulation. Org. Lett. 2013, $15,2510-2513$.

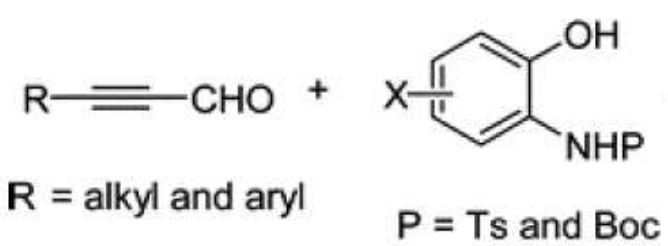

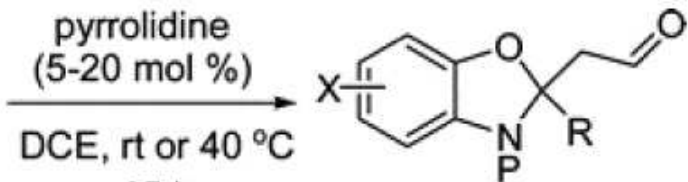

$15 \mathrm{~h}$

$83-95 \%$ yields

Exercise \#145

Yanpeng Xing, Hongyang Zhao, Qiongyi Shang, Jing Wang, Ping Lu, and Yanguang Wang. Parallel Copper Catalysis: Diastereoselective Synthesis of Polyfunctionalized Azetidin-2-imines. Org. Lett. 2013, 15, 2668-2671. 
$2 \sum^{\mathrm{R}^{1}}+\mathrm{R}^{2} \mathrm{SO}_{2} \mathrm{~N}_{3}+\underbrace{\mathrm{R}^{4}}_{\mathrm{Cl}^{3}} \frac{\mathrm{Cul}, \mathrm{Et}_{3} \mathrm{~N}}{\mathrm{DCM}, \mathrm{rt}, 6 \mathrm{~h}}$

Exercise \#146

Yi-Feng Wang, Feng-Lian Zhang, and Shunsuke Chiba. Oxidative Radical Skeletal Rearrangement Induced by Molecular Oxygen: Synthesis of Quinazolinones. Org. Lett. 2013, 15, 2842-2845.<smiles>[R]C1=NOC(c2ccccc2)N1[R]</smiles>

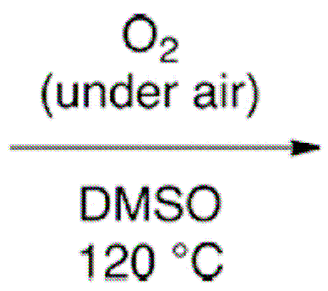<smiles>[R]c1nc2ccccc2c(=O)n1[R]</smiles>

Exercise \#147

Hsuan-Hao Kuan, Chung-Han Chien, and Kwunmin Chen. Synthesis of Fully Substituted Dispirocyclohexanes by Organocatalytic $[2+2+2]$ Annulation Strategy between 2Arylideneindane-1,3-diones and Aldehydes. Org. Lett. 2013, 15, 2880-2883.
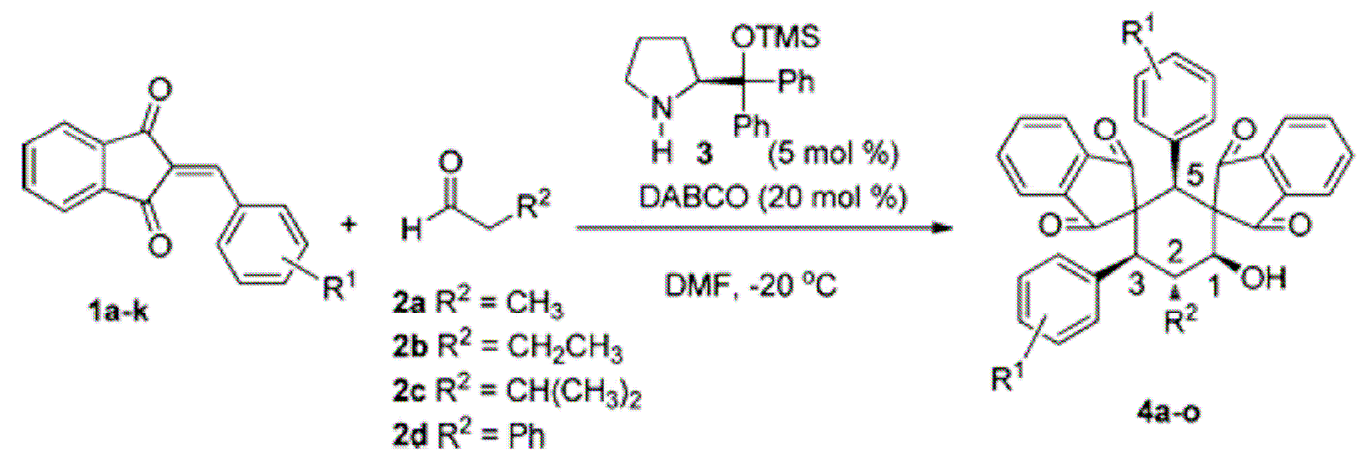

Exercise \#148

Isabelle Aillaud, David M. Barber, Amber L. Thompson, and Darren J. Dixon.

Enantioselective Michael Addition/Iminium Ion Cyclization Cascades of TryptamineDerived Ureas. Org. Lett. 2013, 15, 2946-2949.

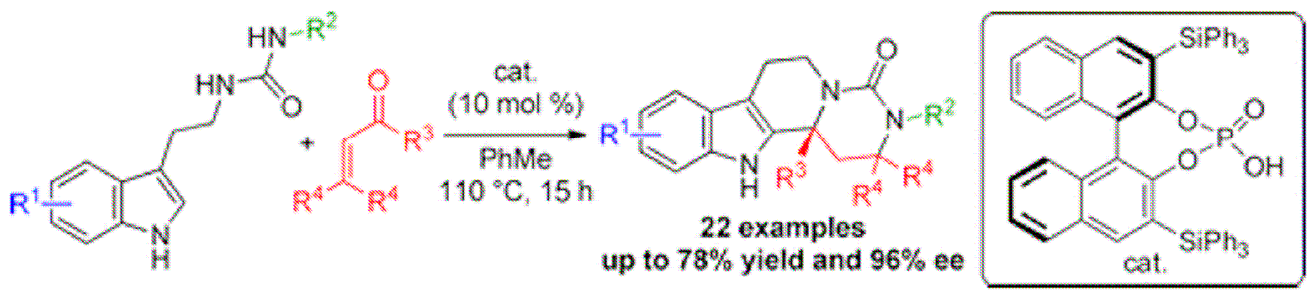


Exercise \#149

Bernardo Pezzati, Mathieu F. Chellat, John J. Murphy, Céline Besnard, Gianna Reginato, John C. Stephens, and Alexandre Alexakis. Organocatalytic Asymmetric Annulation of 1,3-Bis(alkoxycarbonyl)buta-1,3-dienes and Aldehydes. Org. Lett. 2013, 15, 2950-2953.<smiles>[R]C=C(C=CC(=O)O)C(=O)O[2H]</smiles>

$1 a-\mathbf{i}$<smiles>O=CCP</smiles>

2a-f

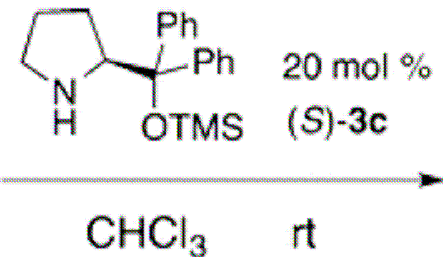

E/Zmixtures

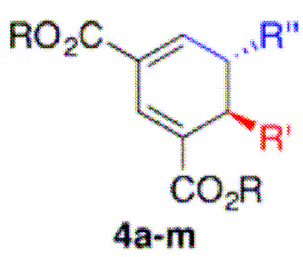

yields up to $96 \%$ ee up to $99.5 \%$ dr up to $20 / 1$

Exercise \#150

Yuzhu Yang, Jinzhong Yao, and Yuhong Zhang. Synthesis of Polysubstituted Furans via Copper-Mediated Annulation of Alkyl Ketones with $\alpha, \beta$-Unsaturated Carboxylic Acids. Org. Lett. 2013, 15, 3206-3209.

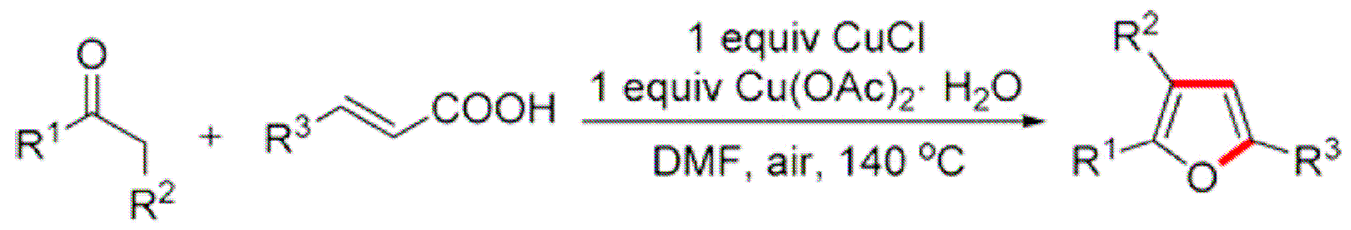

$\mathrm{R}^{1}=$ aryl, heteroaryl, $t-\mathrm{Bu}$

$\mathrm{R}^{2}=\mathrm{H}, \mathrm{Me}, \mathrm{Et}, n-\mathrm{Bu}, \mathrm{Ph}$

$\mathrm{R}^{3}=$ aryl, heteroaryl, styryl

\section{Exercise \#151}

Donald R. Wenz and Javier Read de Alaniz. Aza-Piancatelli Rearrangement Initiated by Ring Opening of Donor-Acceptor Cyclopropanes. Org. Lett. 2013, 15, 3250-3253.

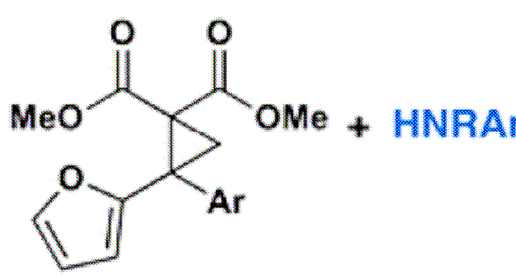

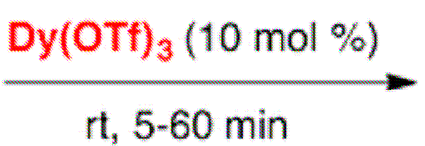<smiles>[R]N([Al])[C@@H]1C=CC(=O)[C@@]1(CC(C(C)=O)C(C)=O)C(C)=O</smiles>

\section{Exercise \#152}

Shengjun Ni, Jie Chen, and Shengming Ma. Unexpected Regioselectivity Switch: Organophosphine-Triggered Reactions of Cyclopropene-1,1-dicarboxylates with Aldehydes. Org. Lett. 2013, 15, 3290-3298. 


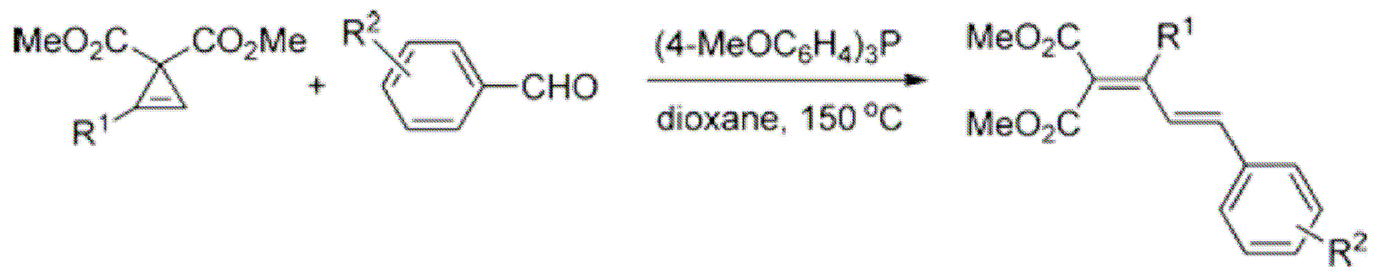

Exercise \#153

Sara Meninno, Gianluca Croce, and Alessandra Lattanzi. Asymmetric Synthesis of Trisubstituted Tetrahydrothiophenes Bearing a Quaternary Stereocenter via Double Michael Reaction Involving Dynamic Kinetic Resolution. Org. Lett. 2013, 15, 34363439 .

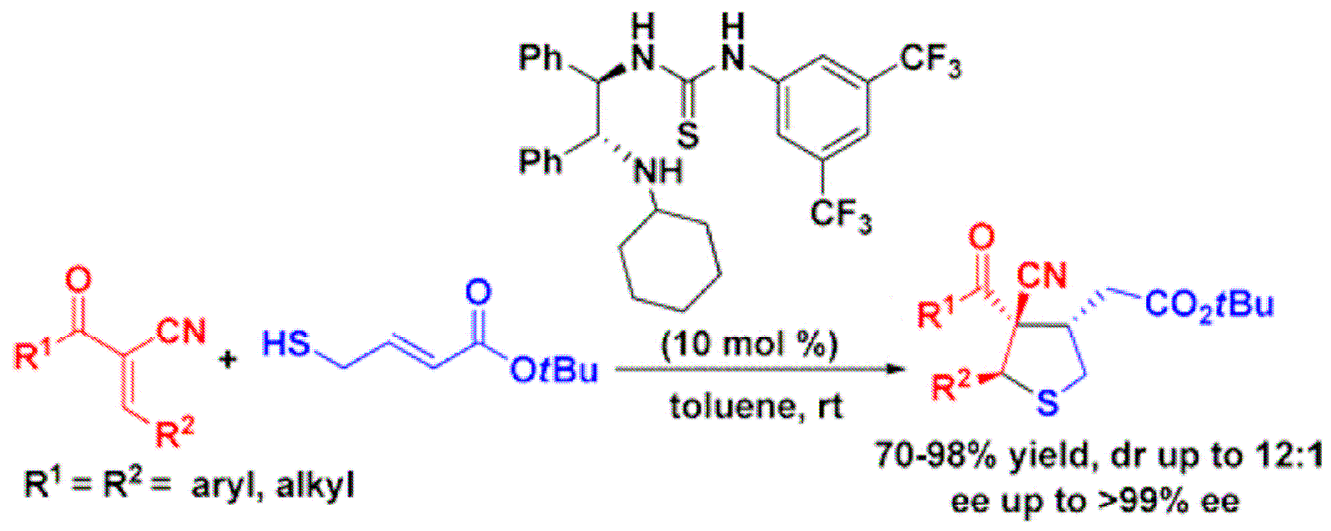

Exercise \#154

Min Yang, Jie Tang, and Renhua Fan. Dearomatization Strategy of $\beta$-Enamino Ester: Construction of Indenoazepines via Tandem Michael Addition/Polycyclization. Org. Lett. 2013, 15, 3464-3467.<smiles>[R]C#Cc1cc([R3])ccc1N/C([R7])=C\C(=O)[O-]</smiles>

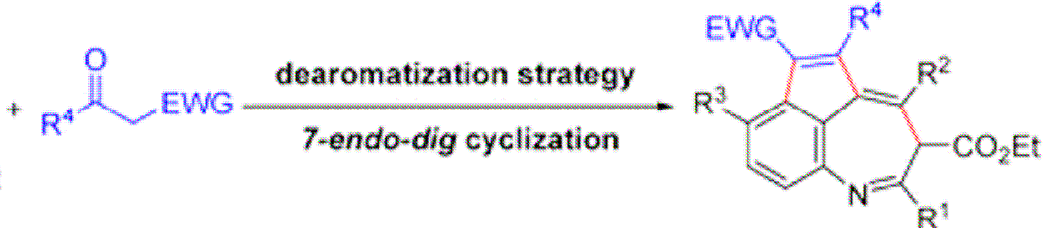

Exercise \#155

Peng Wang, Saihu Liao, Jian-Bo Zhu, and Yong Tang. Iron-Catalyzed ThreeComponent Reaction: Multiple $\mathrm{C}-\mathrm{C}$ Bond Cleavages and Reorganizations. Org. Lett. 2013, 15, 3606-3609. 

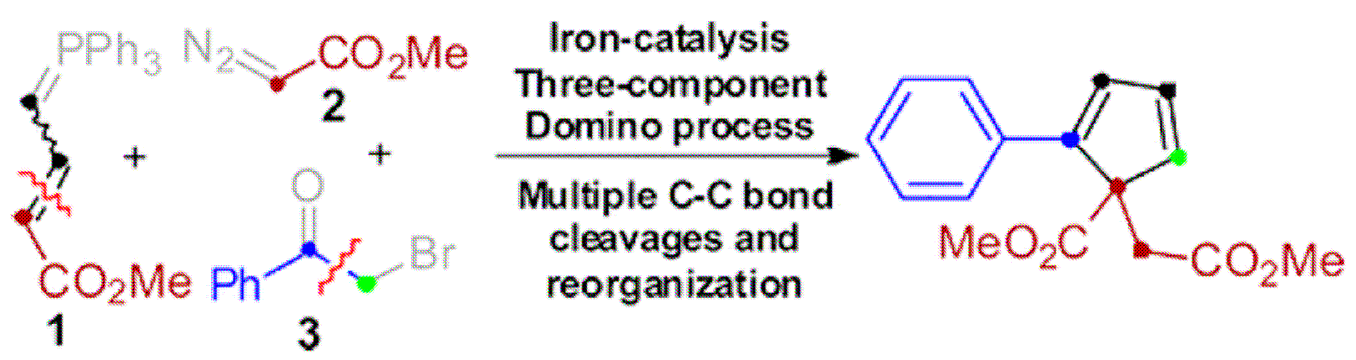

Exercise \#156

Takumi Abe, Shuuhei Nakamura, Reiko Yanada, Tominari Choshi, Satoshi Hibino, and Minoru Ishikura. One-Pot Construction of 3,3'-Bisindolylmethanes through Bartoli Indole Synthesis. Org. Lett. 2013, 15, 3622-3625.<smiles>[R]C(c1c[nH]c2c(Br)cccc12)c1c[nH]c2c(Br)cccc12</smiles>

Exercise \#157

Dimitris Kalaitzakis, Tamsyn Montagnon, Eirini Antonatou, and Georgios

Vassilikogiannakis. One-pot Synthesis of the Tetracyclic Framework of the Aromatic Erythrina Alkaloids from Simple Furans. Org. Lett. 2013, 15, 3714-3717.

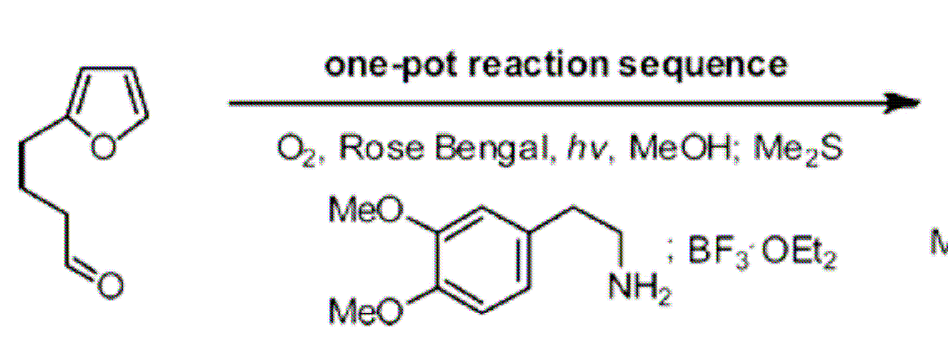<smiles>COc1cc2c(cc1OC)[C@]13CCC[C@@H](O)[C@H]1CC(=O)N3CC2</smiles>

Exercise \#158

Bo Chen and Shengming Ma. Tandem Michael Addition/Cyclization Reaction of 2,3Allenoates with Organozincs: Facile Synthesis of Isocoumarins. Org. Lett. 2013, 15, 3884-3887. 


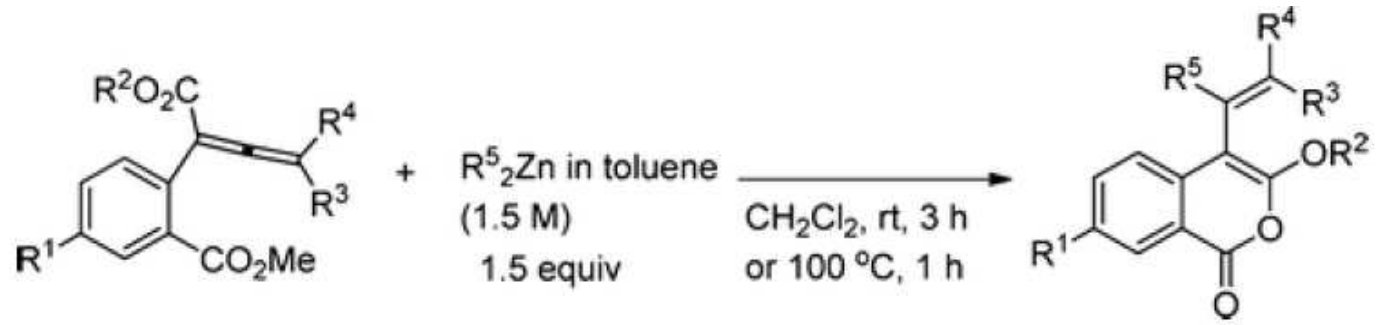

Exercise \#159

Huy H. Nguyen, Teresa A. Palazzo, and Mark J. Kurth. Facile One-Pot Assembly of Imidazotriazolobenzodiazepines via Indium(III)-Catalyzed Multicomponent Reactions. Org. Lett. 2013, 15, 4492-4495.

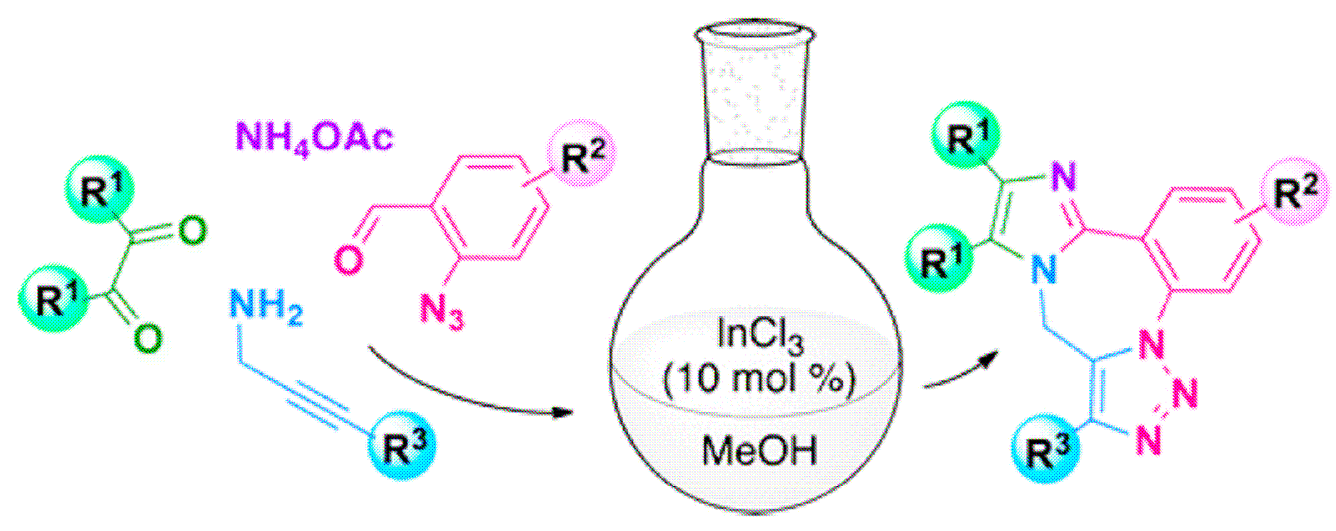

Exercise \#160

Jessica Labarre-Lainé, Redouane Beniazza, Valérie Desvergnes, and Yannick Landais. Convergent Access to Bis-spiroacetals through a Sila-Stetter-Ketalization Cascade. Org. Lett. 2013, 15, 4706-4709.

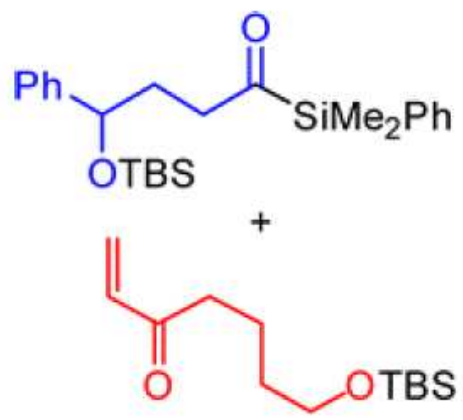<smiles>CC[n+]1csc(CCO)c1C</smiles><smiles>c1ccc(C2CCC3(CCC4(CCCCO4)O3)O2)cc1</smiles>

Exercise \#161

Yong Wang, Chao Chen, Shu Zhang, Zhenbang Lou, Xiang Su, Lirong Wen, and Ming Li. A Concise Construction of Polycyclic Quinolines via Annulation of $\omega$-Cyano-1alkynes with Diaryliodonium Salts. Org. Lett. 2013, 15, 4794-4797. 


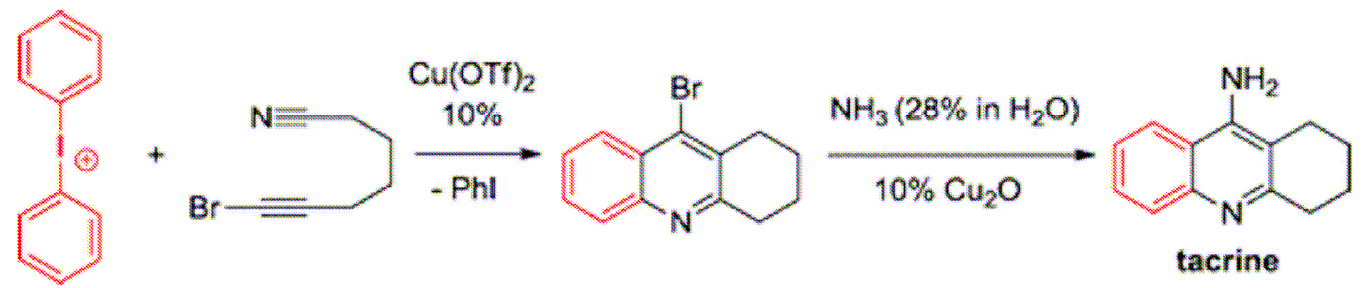

Exercise \#162

Ganesan Bharathiraja, Sekarpandi Sakthivel, Mani Sengoden, and Tharmalingam Punniyamurthy. A Novel Tandem Sequence to Pyrrole Syntheses by 5-endo-dig Cyclization of 1,3-Enynes with Amines. Org. Lett. 2013, 15, 4996-4999.

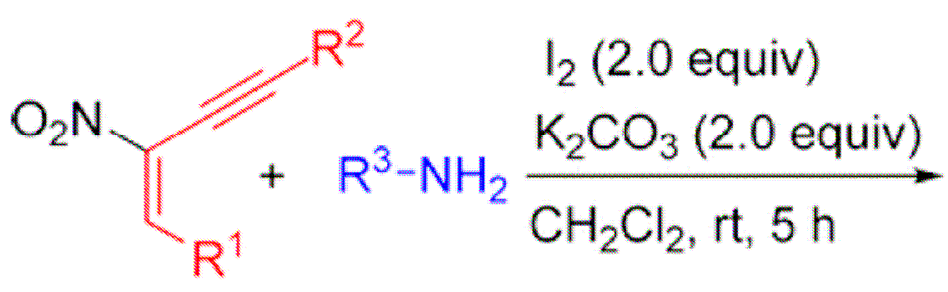

Exercise \#163 One-Pot Sequential [3 + 2]/[3 + 2] Annulation of Allenoates with Cyclic Ketimines. Org. Lett. 2013, 15, 5214-5217.

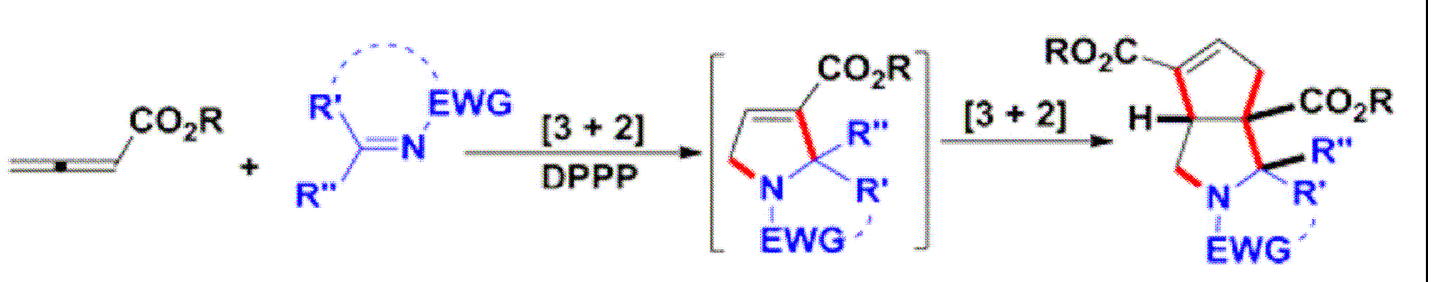

Exercise \#164

Asunción Barbero, Alberto Diez-Varga, and Francisco J. Pulido. Multicomponent Prins Cyclization from Allylsilyl Alcohols Leading to Dioxaspirodecanes. Org. Lett. 2013, 15, 5234-5237. 

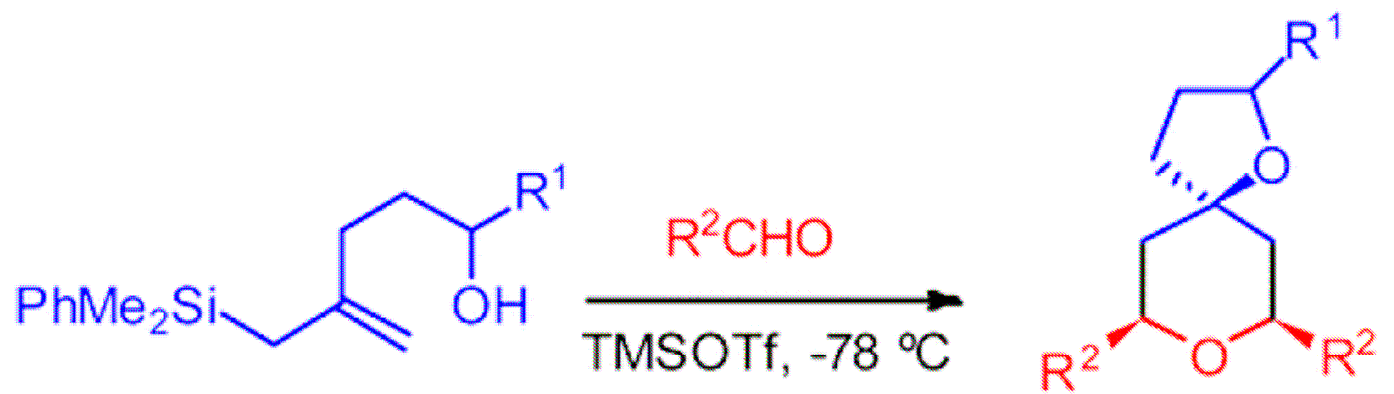

Exercise \#165

Martin Déry, Louis-Philippe D. Lefebvre, Kevin Aissa, and Claude Spino. N-

Heteropolycyclic Compounds from the Formal Intramolecular $(4+1)$-Cycloaddition of Chromium Aminocarbenes. Org. Lett. 2013, 15, 5456-5459.<smiles>CC(=O)OC(C)=CC=CCCCN(C=CC(=O)OCc1ccccc1)CC1CCCCC1</smiles>

14

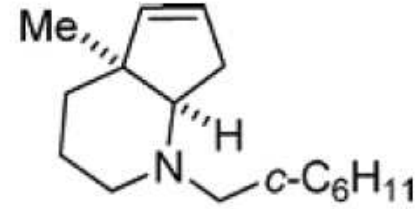

15

Exercise \#166

Xin Fang, Jun Li, Hai-Yan Tao, and Chun-Jiang Wang. Highly Diastereoselective DABCO-Catalyzed [3 + 3]-Cycloaddition of 1,4-Dithiane-2,5-diol with Azomethine Imines. Org. Lett. 2013, 15, 5554-5557.<smiles></smiles>

Exercise \#167

Ming Chen, Ning Sun, and Yuanhong Liu. Environmentally Benign Synthesis of Indeno[1,2-b] quinolines via an Intramolecular Povarov Reaction. Org. Lett. 2013, 15, 5574-5577. 


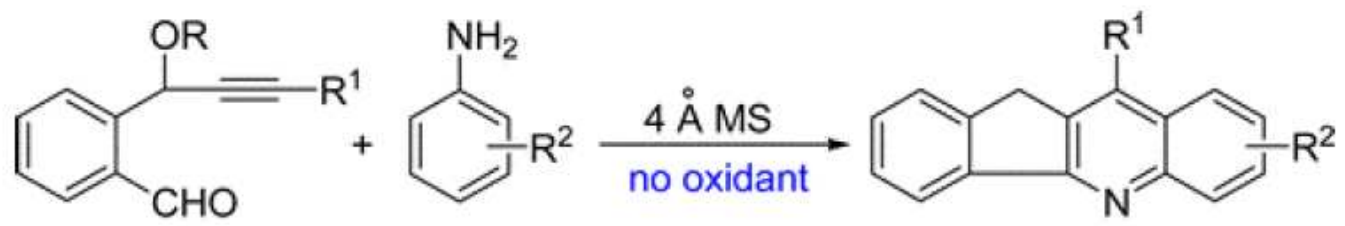

\section{$\mathrm{R}=\mathrm{Piv}$ or $\mathrm{Bz} \quad$ via intramolecular Povarov reaction 21 examples}

\section{Exercise \#168}

Paramita Das, Suman Ray, and Chhanda Mukhopadhyay. Exploitation of Dual Character of CN Moiety in the Synthesis of Uniquely Decorated 3H-Pyrroles: A Rare Observation. Org. Lett. 2013, 15, 5622-5625.

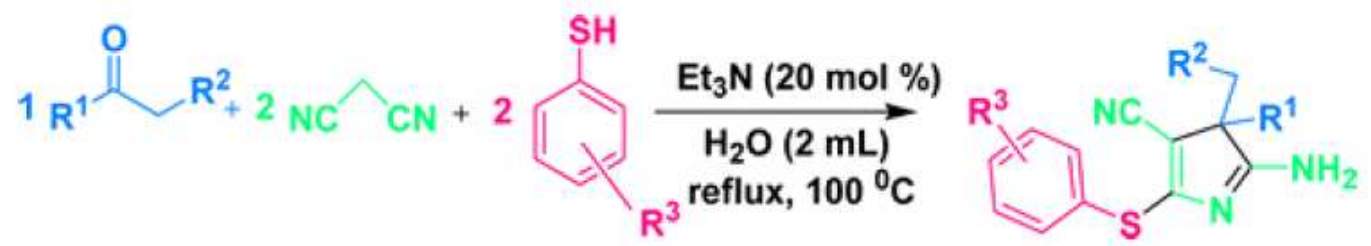

Exercise \#169

Sheng-Chieh Chuang, Parthasarathy Gandeepan, and Chien-Hong Cheng. Synthesis of Isoquinolines via Rh(III)-Catalyzed C-H Activation Using Hydrazone as a New Oxidizing Directing Group. Org. Lett. 2013, 15, 5750-5753.<smiles>[R]C#C[R]([R20])C(=O)OC</smiles>

Exercise \#170

Fenguo Zhou, Xu Liu, Ning Zhang, Yongjiu Liang, Rui Zhang, Xiaoqing Xin, and Dewen Dong. Copper-Catalyzed Three-Component Reaction: Solvent-Controlled Regioselective Synthesis of 4-Amino- and 6-Amino-2-iminopyridines. Org. Lett. 2013, 15, 5786-5789. 


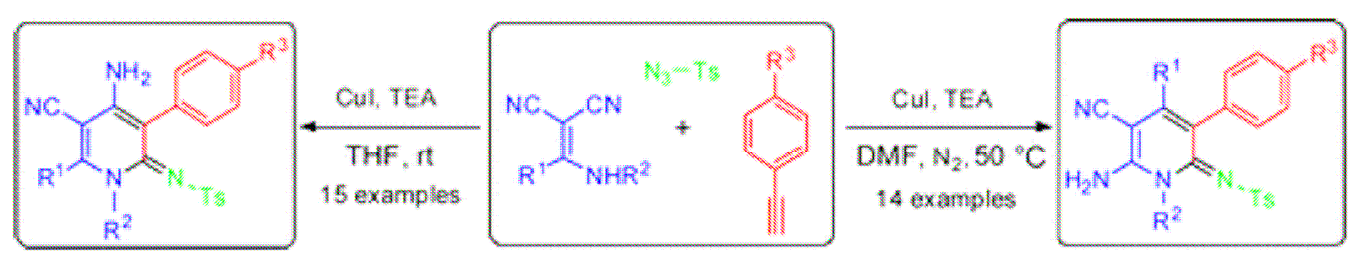

Exercise \#171

Valentina Mercalli, Fiorella Meneghetti, and Gian Cesare Tron. Isocyanide-Mediated Multicomponent Synthesis of C-Oximinoamidines. Org. Lett. 2013, 15, 5902-5905.<smiles>[R2]N=C([R])C(=N[R2])C([R])=NO</smiles>

Exercise \#172

Guolin Zhang, Hangcheng Ni, Wenteng Chen, Jiaan Shao, Huan Liu, Binhui Chen, and Yongping Yu. One-Pot Three-Component Approach to the Synthesis of Polyfunctional Pyrazoles. Org. Lett. 2013, 15, 5967-5969.<smiles>[R]C=C([R2])[NH2+][R]C=O</smiles>

Exercise \#173

Hui-Ying Yin, Reactions between 2,3-Dihydroinden-1-ones and Aryl Chlorides Catalyzed by an $\mathrm{N}$ Heterocyclic Carbene-Palladium(II)-1-Methylimidazole Complex. Org. Lett. 2013, 15, 6042-6045.

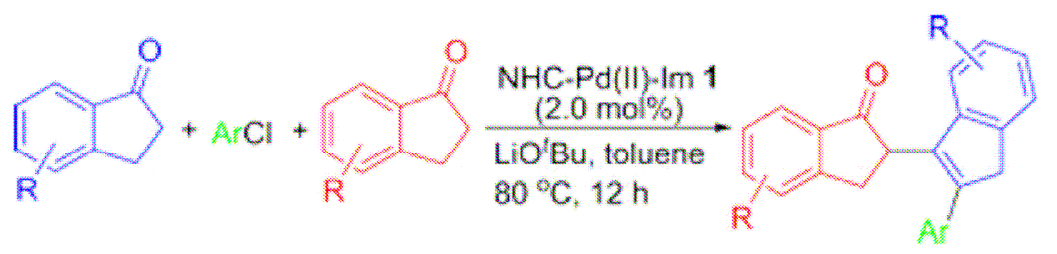

20 examples $54-95 \%$ yield 


\section{Exercise \#174}

Chung-Yu Chen, Wan-Ping Hu, Pi-Cheng Yan, Gopal Chandru Senadi, and Jeh-Jeng Wang. Metal-Free, Acid-Promoted Synthesis of Imidazole Derivatives via a

Multicomponent Reaction. Org. Lett. 2013, 15, 6116-6119.

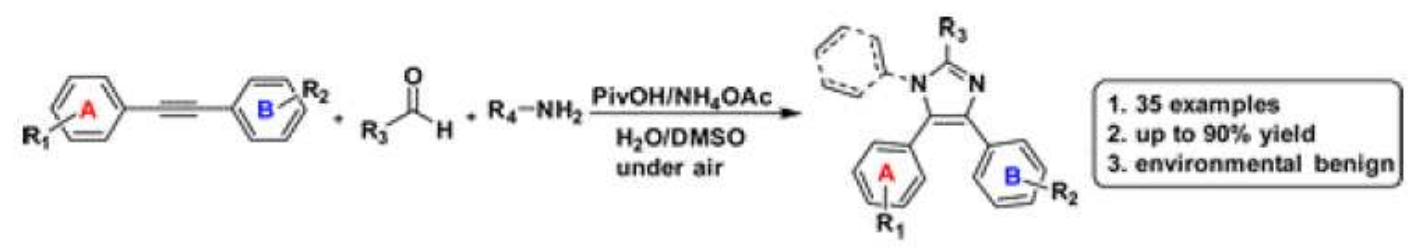

Exercise \#175

Jihui Li and Luc Neuville. Copper-Catalyzed Oxidative Three-Component Synthesis of $N$, $N^{\prime}, N^{\prime}$-Trisubstituted Guanidines. Org. Lett. 2013, 15, 6124-6127.

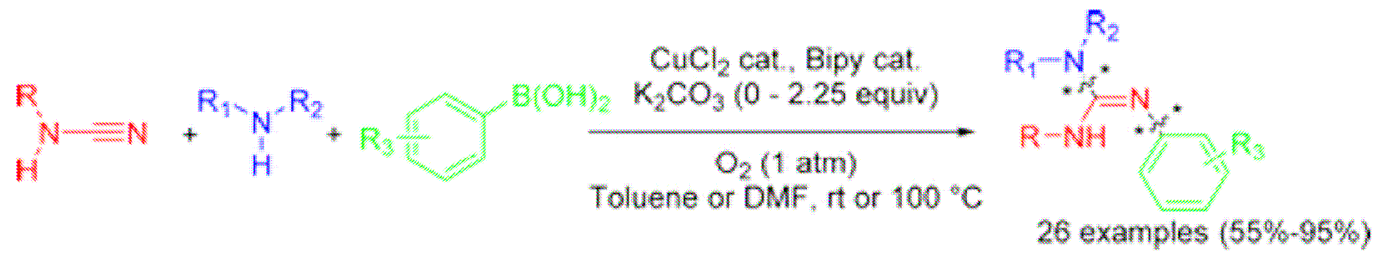

Exercise \#176

C. R. Sinu, Eringathodi Suresh, and Vijay Nair. N-Heterocyclic Carbene Catalyzed Reaction of Cinnamils Leading to the Formation of 2,3,8-Triaryl Vinyl Fulvenes: An Uncommon Transformation. Org. Lett. 2013, 15, 6230-6233.<smiles>[R]C=CC(=O)C(=O)C=C[R]</smiles>

Exercise \#177

Bor-Cherng Hong, Cheng-Wei Lin, Wei-Kai Liao, and Gene-Hsiang Lee. Sequential Asymmetric Catalysis in Michael-Michael-Michael-Aldol Reactions:

Merging Organocatalysis with Photoredox Catalysis in a One-Pot Enantioselective Synthesis of Highly Functionalized Decalines Bearing a Quaternary Carbon Stereocenter Org. Lett. 2013, 15, 6258-6261. 

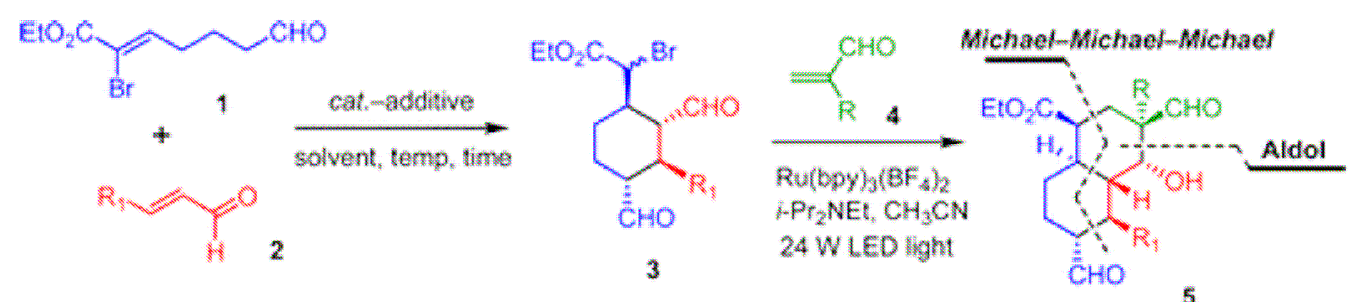

\section{Ring construction and mapping exercises}

For each reaction shown from table of contents graphics taken directly as depicted in the literature, redraw the reaction using the principle of conservation of structural aspect applied to the product structures shown. Highlight the target bonds made in the product structures and balance the equations showing by-products. Describe the ring construction strategy and mapping.

\section{Exercise \#1}

Zeng, F.; Alper, H. Org. Lett. 2010, 12, 1188.<smiles>N=C=Nc1ccccc1I</smiles><smiles>[R10]OC(=O)O[R10]([H])([H])[H]</smiles><smiles>Nc1nc2ccccc2c(=O)n1Br</smiles>

Exercise \#2

Liang, H.; Ciufolini, M.A. Org. Lett. 2010, 12, 1760.<smiles>C=C/C=C\C(=O)N[C@H](CO)CCc1ccc(O)cc1</smiles>

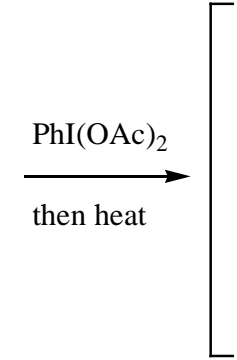

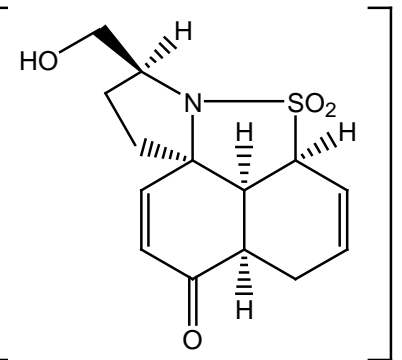

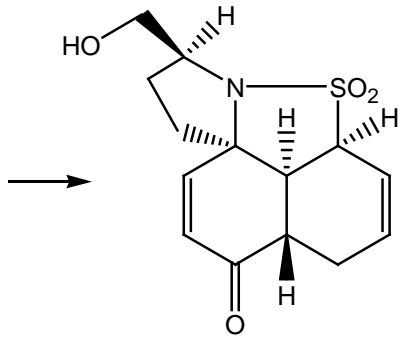

Exercise \#3

Lee, H.Y.; Jung, Y.; Yoon, Y.; Kim , B.G.; Kim, Y. Org. Lett. 2010, 12, 2672. 

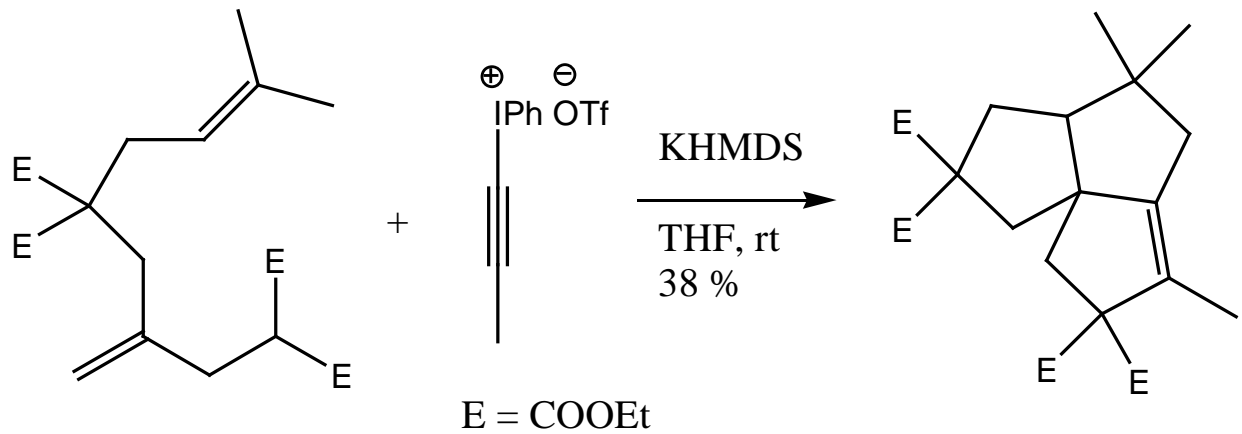

Exercise \#4

Ramazani, A.; Rezaei, A. Org. Lett. 2010, 12, 2852.

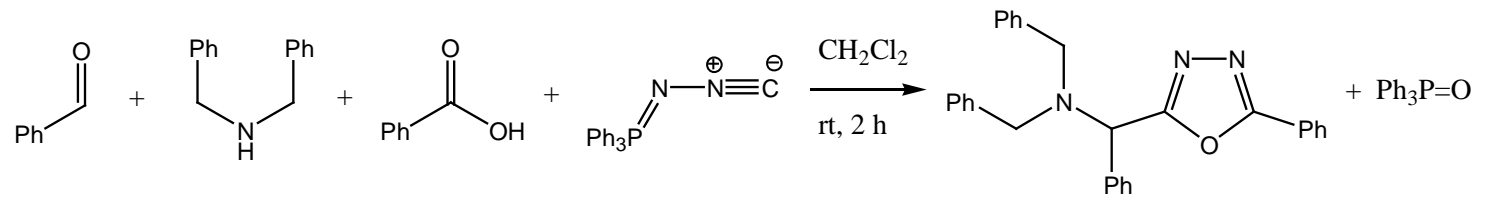

Exercise \#5

Burke, J.P.; Sabat, M.; Iovan, D.A.; Myers, W.H.; Chruma, J.J. Org. Lett. 2010, 12, 3192.<smiles>C=C[C@H]1C=C[C@@H]2CCCC(=O)[C@@]2(C)[C@H]1[C@H](C)CNC(=O)OC(C)(C)C</smiles>

\section{Exercise \#6}

Trofimov, B.A.; Shemyakina, O.A.; Malkina, A.G.; Ushakov, I.A.; Kazheva, O.N.;

Alexandrov, G.G.; Dyachenko, O.A. Org. Lett. 2010, 12, 3200.<smiles>[R]C([R])(O)C#C[NH3+]</smiles>

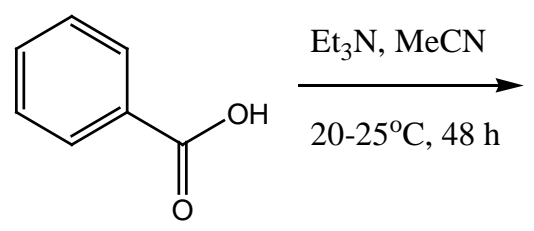<smiles>[R7]C1([R])OC(c2ccccc2)=C(C#N)C1=O</smiles>

Exercise \#7

Zeng, F.; Alper, H. Org. Lett. 2010, 12, 3642. 
<smiles>[R6]c1ccc(N=C=Nc2ccc([R6])cc2I)c(I)c1</smiles>

Exercise \#8

Chen, W.; Hu, M.; Wu, J.; Zou, H.; Yu, Y. Org. Lett. 2010, 12, 3863.

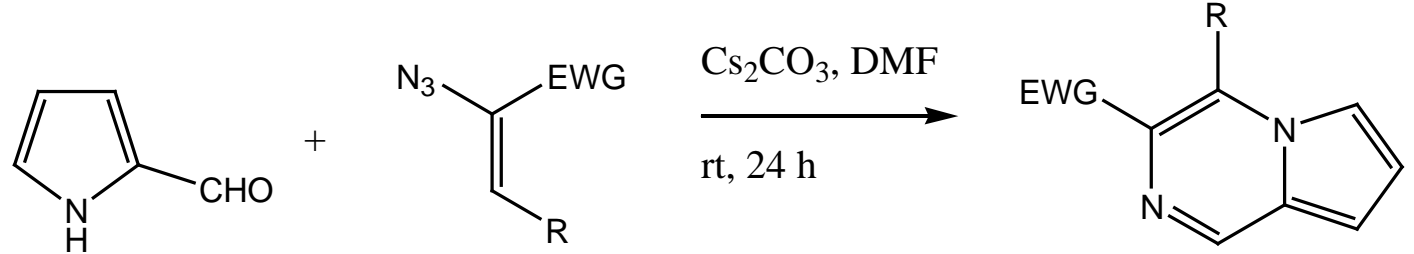

Exercise \#9

Miao, L.; Haque, I.; Manzoni, M.R.; Tham, W.S.; Chemler, S.R. Org. Lett. 2010, 12, 4739 .
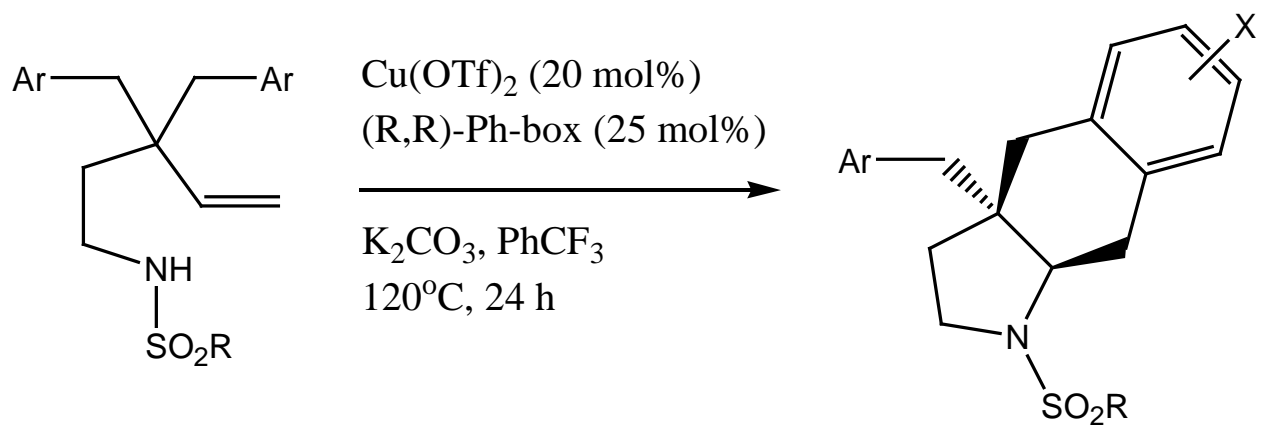

Exercise \#10

Cleary, L.; Yoo, H.; Shea, K.J. Org. Lett. 2011, 13, 1781.<smiles>[R]C=CC([X])=[Y]CCC(=C)C=C</smiles>
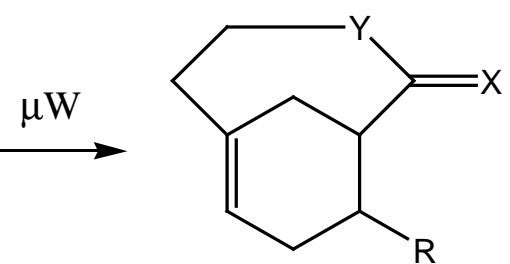

Exercise \#11

Chaloner, P. "Organic Chemistry - a mechanistic approach", CRC Press-Taylor and Francis: Boca Raton, 2015, p. 880. 


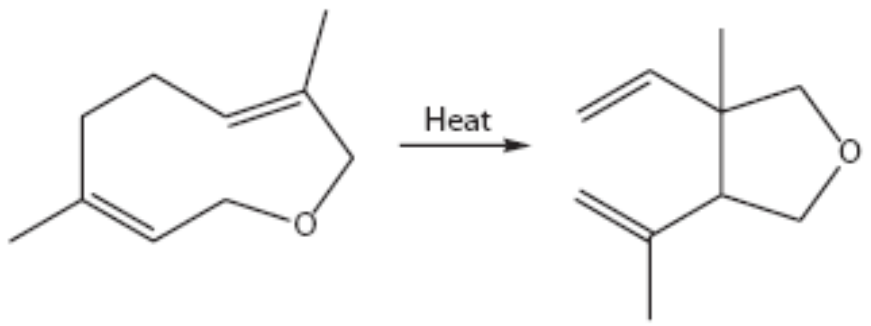

Exercise \#12

Chaloner, P. "Organic Chemistry - a mechanistic approach", CRC Press-Taylor and Francis: Boca Raton, 2015, p. 893.<smiles>C[C@@H]1CCCC[C@]1(O)Cc1ccccc1</smiles>

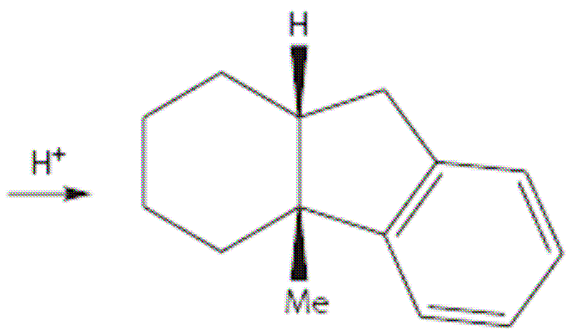

Exercise \#13

Chaloner, P. "Organic Chemistry - a mechanistic approach", CRC Press-Taylor and Francis: Boca Raton, 2015, p. 992.<smiles>CO[N+](Cc1ccccc1)c1ccccc1</smiles>

Exercise \#14

Savin, K.A. "Writing Reaction Mechanisms in Organic Chemistry", $3^{\text {rd }}$ ed., Elsevier: Amsterdam, 2014, p. 436.<smiles>O=C1C=C2C=CC3CC2(O1)C1CCCCN31</smiles>

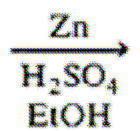<smiles>O=C1Cc2ccccc2C2CCCCN12</smiles> 


\section{$\underline{\text { V. Synthesis schemes exercises }}$}

Redraw the following schemes of synthesis plans according to the principle of conservation of structural aspect. Show all intermediate product structures for all reaction steps.

Exercise \#1

Zhu, H.J. "Organic Stereochemistry - experimental and computational methods", WileyVCH: Weinheim, 2015, p. 287.

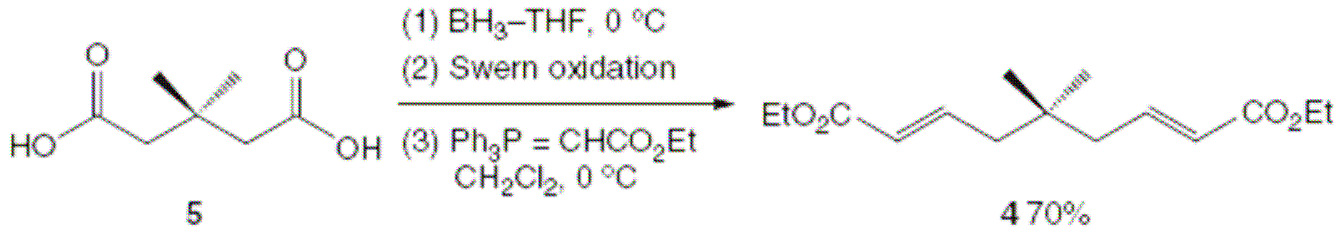<smiles>COC(=O)C[C@H]1CC(C)(C)C[C@H]1CC(=O)O[Na]</smiles>

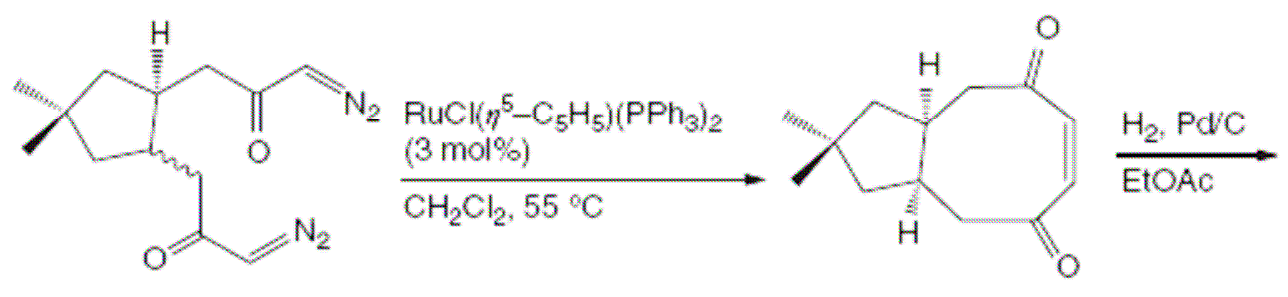

$873 \%$<smiles>CC1(C)C[C@H]2CC(=O)CCC(=O)C[C@H]2C1</smiles>

$3100 \%$

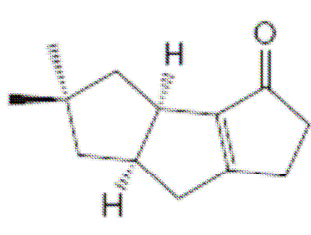

$1199 \%$

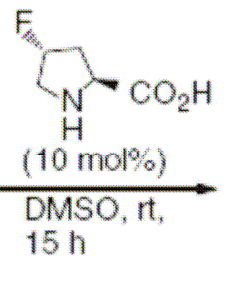

DMSO
$15 \mathrm{~h}$

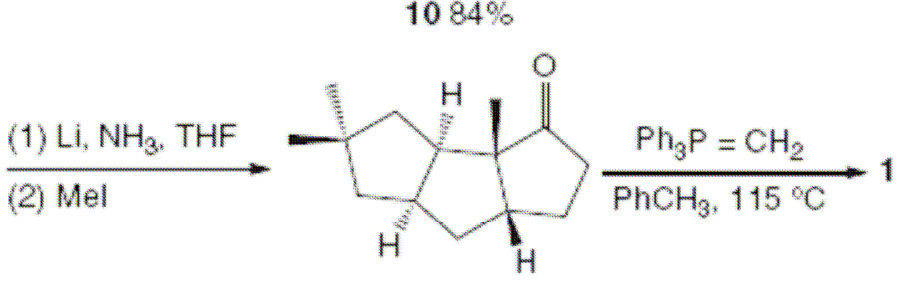

Scheme 10.6 Total synthesis of $(+)$-hirsutene (1). 


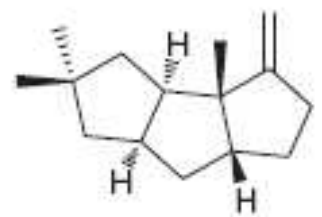

(t)- Hirsutene 1

Exercise \#2

Zhu, H.J. "Organic Stereochemistry - experimental and computational methods", WileyVCH: Weinheim, 2015, p. 288.<smiles>C=C(C)[C@H]1CC[C@@H](C)C(=O)C1</smiles>

$(2 R, 5 R)-20$

(1) $\mathrm{O}_{3} / \mathrm{MeOH}-78^{\circ} \mathrm{C}$

(2) $\mathrm{Cu}(\mathrm{OAC})_{2}-\mathrm{H}_{2} \mathrm{O},-20^{\circ} \mathrm{C}$

(3) $\mathrm{FeSO}_{4}-7 \mathrm{H}_{2} \mathrm{O},-20^{\circ} \mathrm{C}$<smiles>CC1CCC=CC1=O</smiles>

$1942 \%$

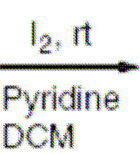

DCM<smiles>C[C@H]1CCC=C(I)C1=O</smiles>

$2167 \%$

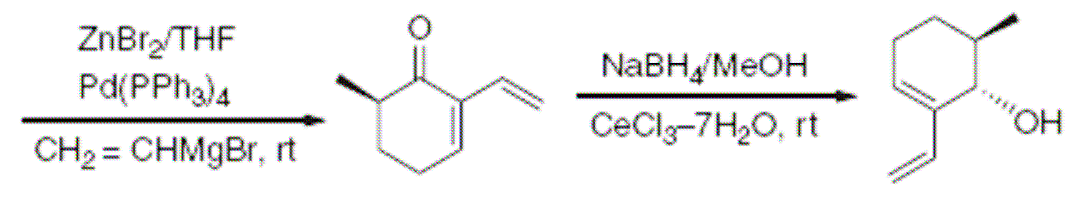

$2268 \%$

$1762 \%$

Scheme 10.8 Synthesis of intermediate 17 from the natural material $(2 R, 5 R)-(+)-$ dihydrocarvone.

Exercise \#3

Zhu, H.J. "Organic Stereochemistry - experimental and computational methods”, WileyVCH: Weinheim, 2015, p. 291. 

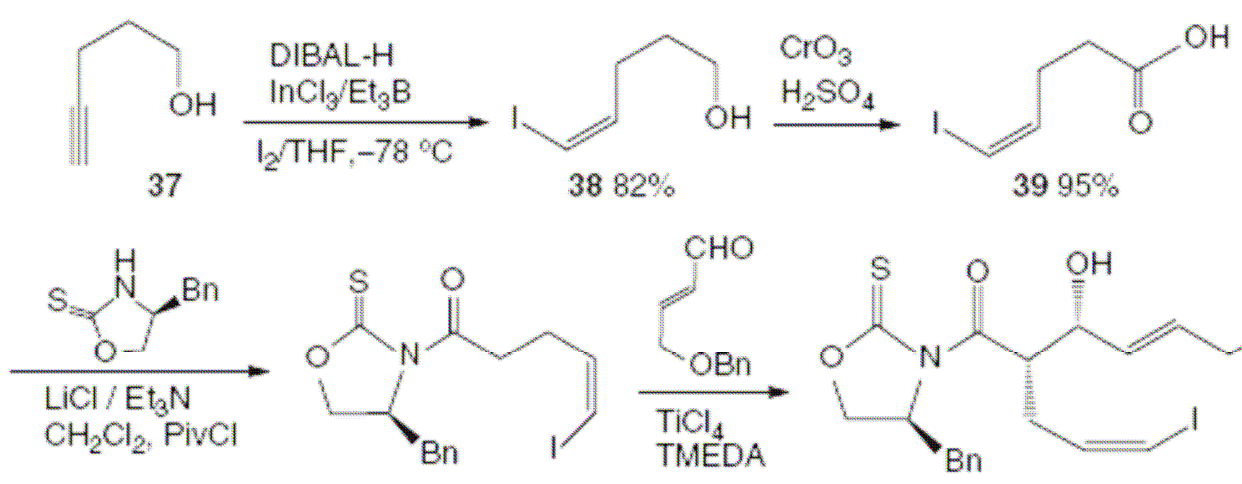

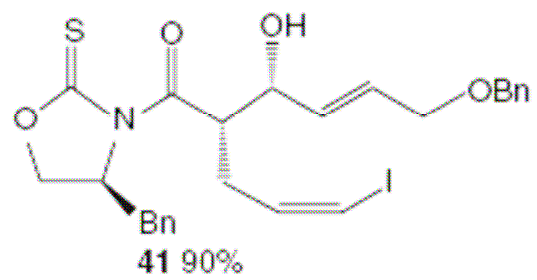<smiles>CCCCO[C@@H](/C=C/COCc1ccccc1)[C@@H](C/C=C\I)C(=O)N1C(=S)OC[C@H]1Cc1ccccc1</smiles>

$4193 \%$

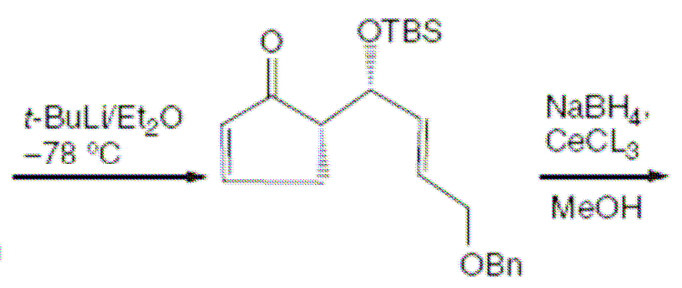

$4275 \%$

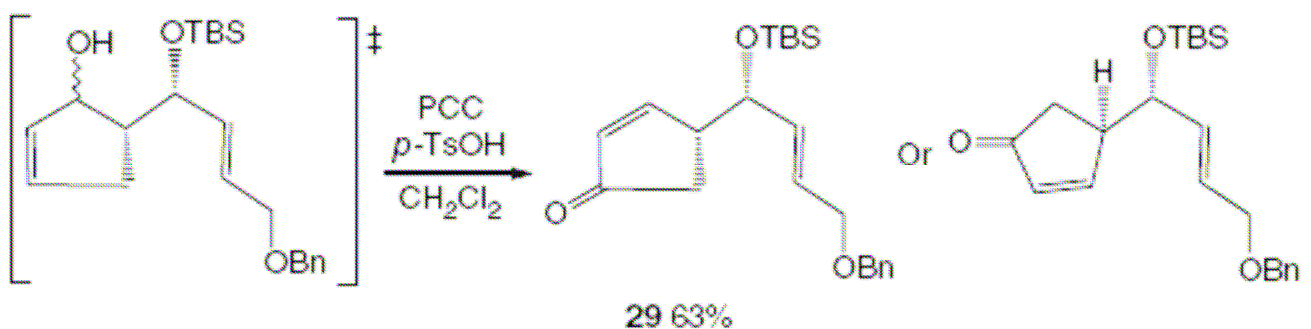

Scheme 10.12 Synthesis of the intermediate 29.

Exercise \#4

Zhu, H.J. "Organic Stereochemistry - experimental and computational methods", WileyVCH: Weinheim, 2015, p. 292. 


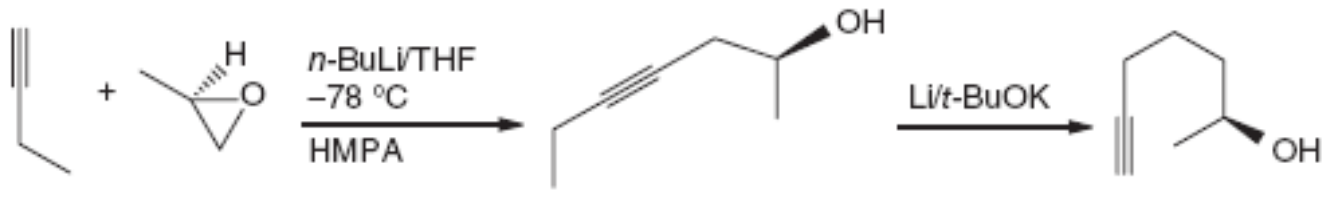

35

36

$4396 \%$

$4489 \%$

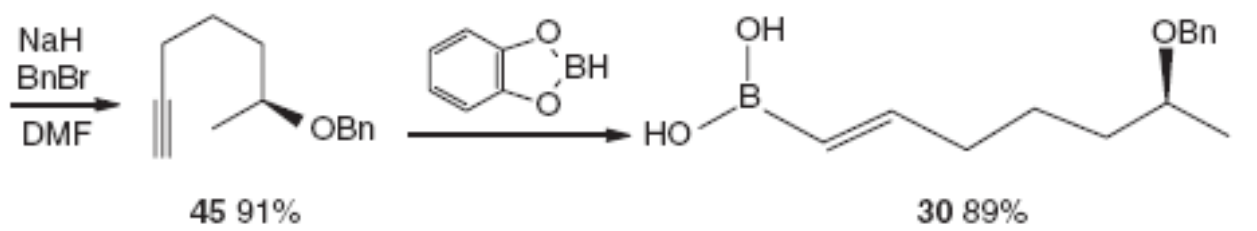

Scheme 10.13 Synthesis of the intermediate 30.

\section{Example \#5}

Kananovich, D.G.; Zubritsky, D.M.; Kulinkovich, O.G. Synlett 2010, 1043.
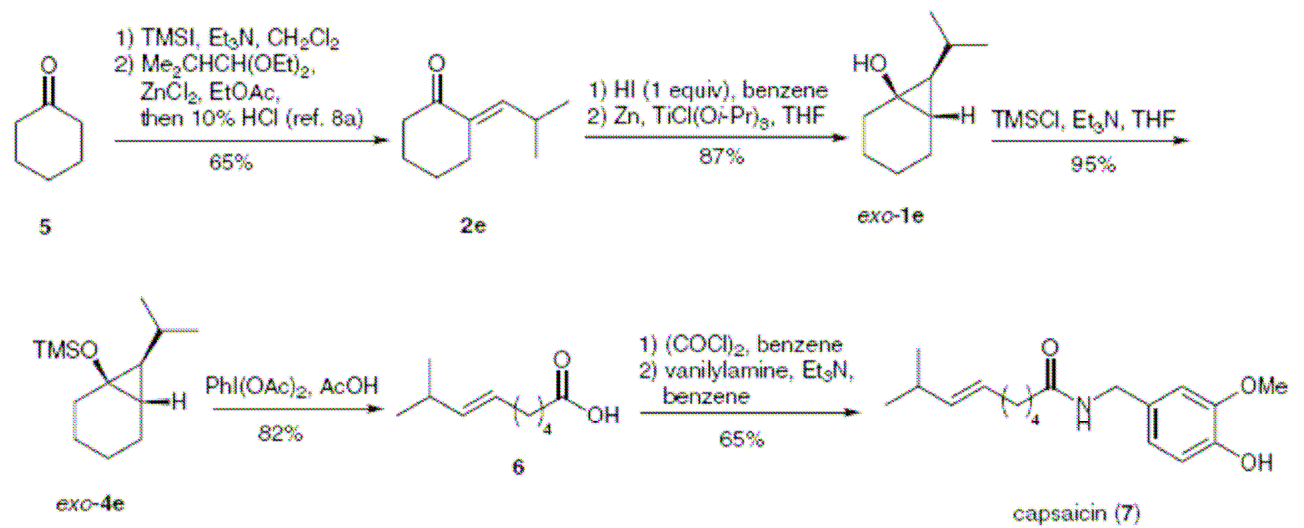

Example \#6

Li, J.; Eastgate, M.D. Org. Biomol. Chem. 2015, 13, 7164. 
(1)

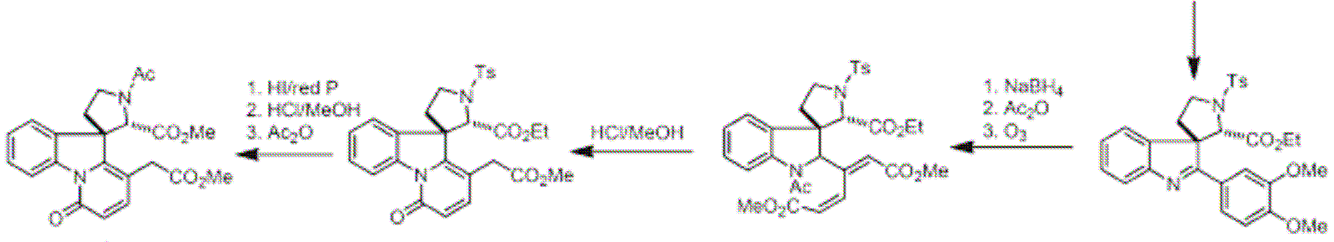

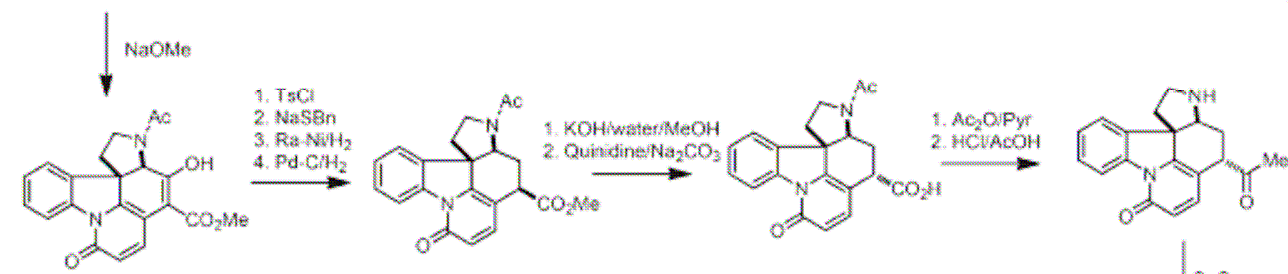

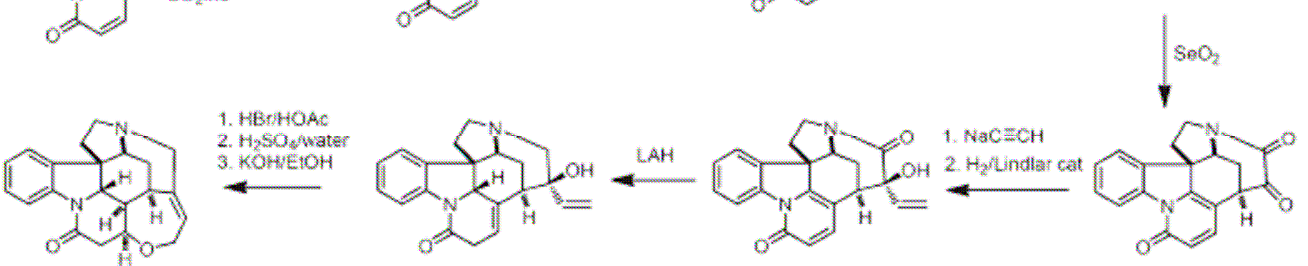

\section{Example \#7}

Li, J.; Eastgate, M.D. Org. Biomol. Chem. 2015, 13, 7164.

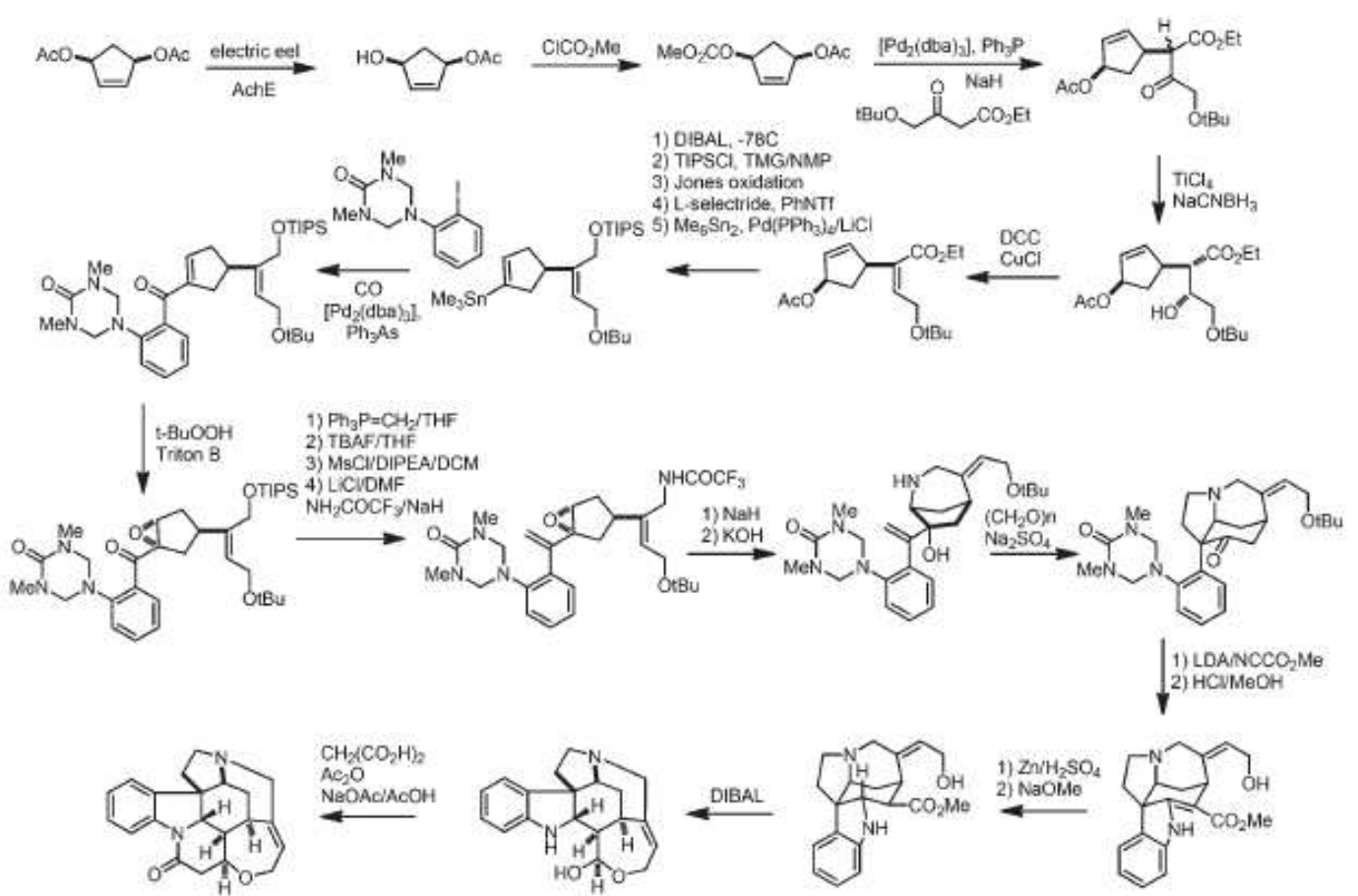

\section{Example \#8}

Li, J.; Eastgate, M.D. Org. Biomol. Chem. 2015, 13, 7164. 

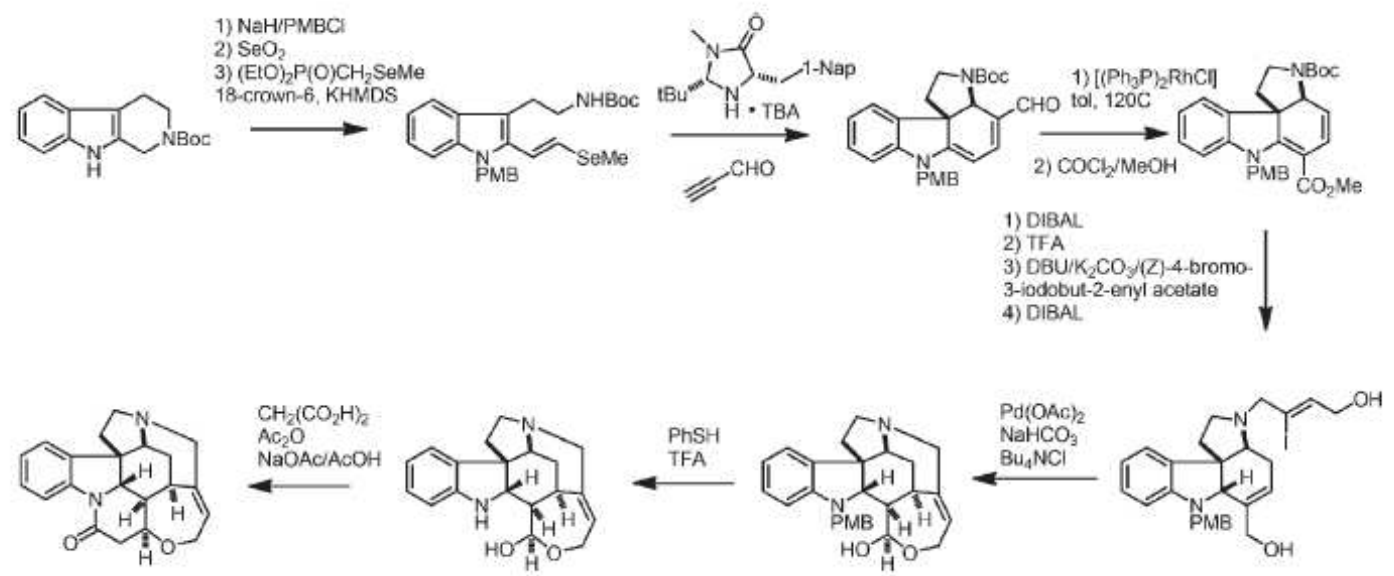

\section{Example \#9}

Li, J.; Eastgate, M.D. Org. Biomol. Chem. 2015, 13, 7164.
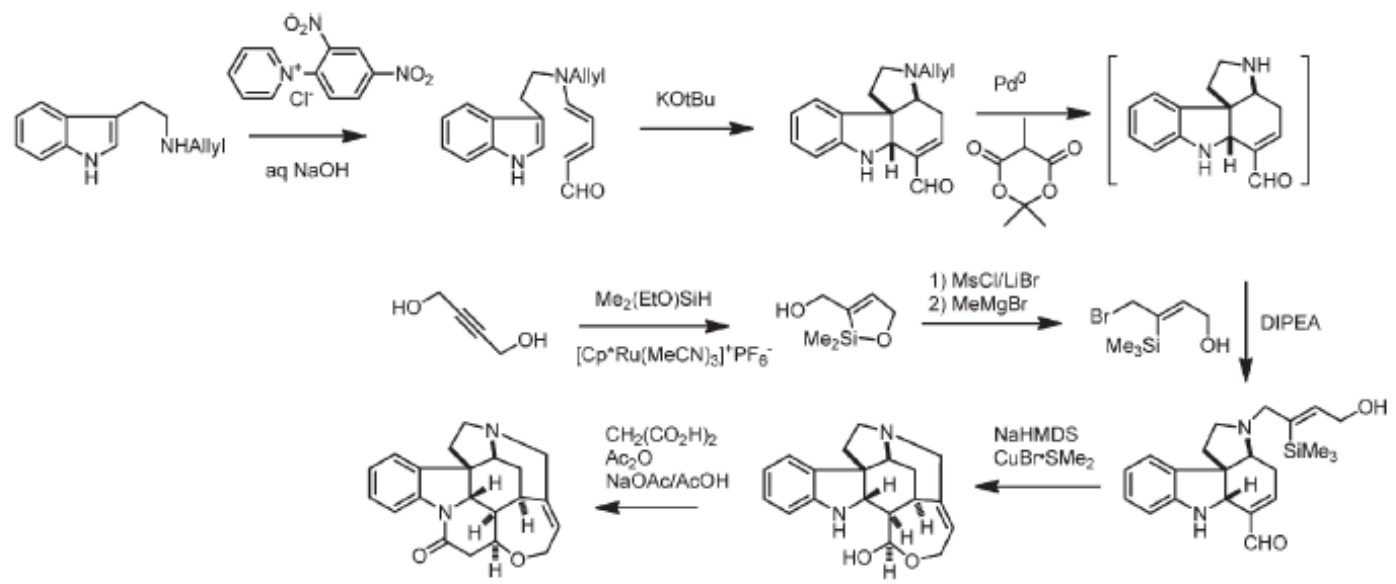

(4)Strychnine

\section{Example \#10}

Li, J.; Eastgate, M.D. Org. Biomol. Chem. 2015, 13, 7164. 


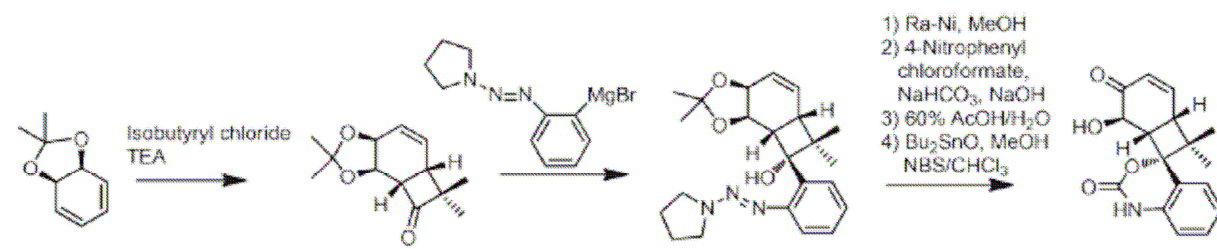

1) Tipsorf
2.6 futidine 2) LHMOS

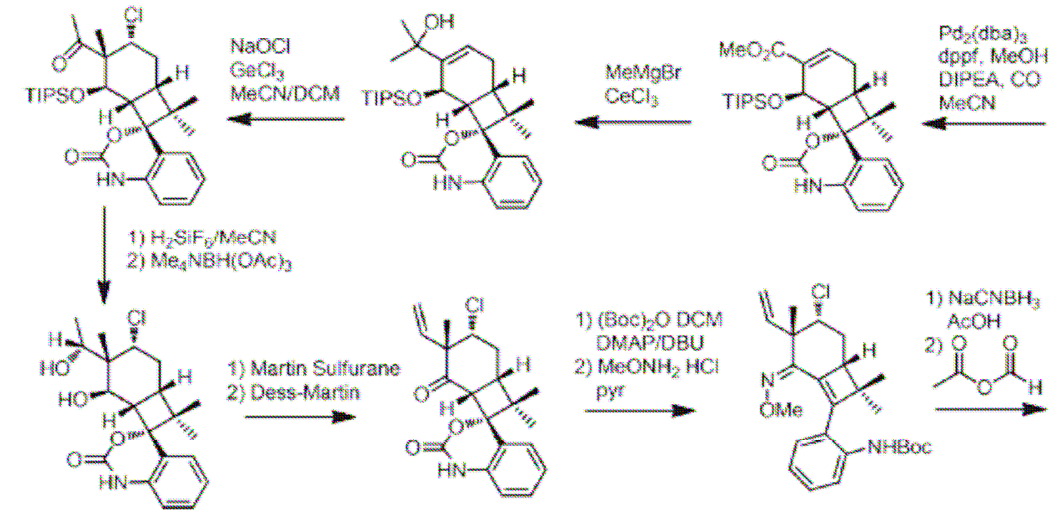
$\checkmark$ PhNTf $_{2}$
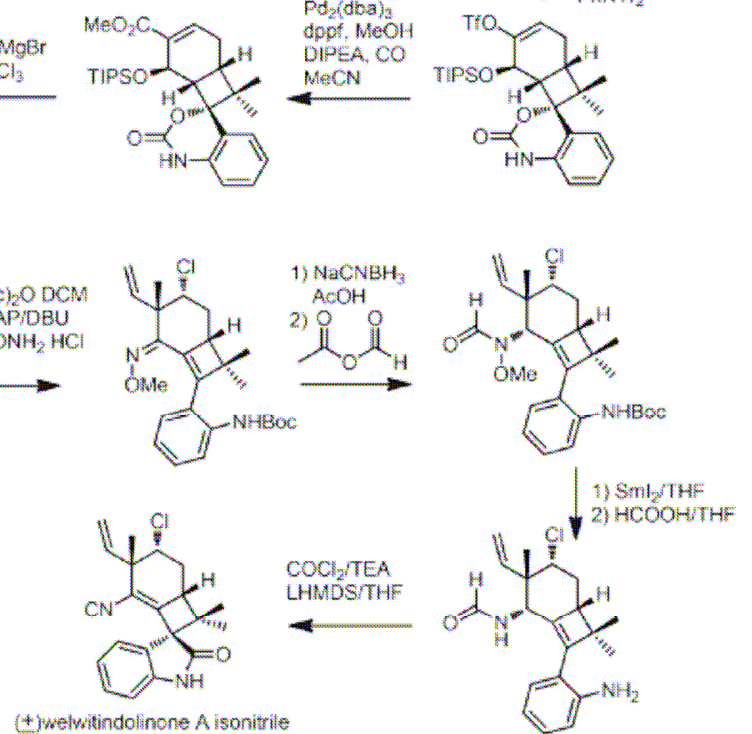

+ iwelwitindolinone A isonitrile

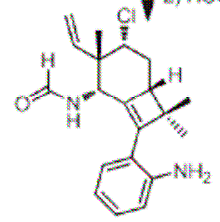


